Physics

Physics Research Publications

Polarimetric observations of 15 active galactic nuclei at high frequencies: Jet kinematics from bimonthly monitoring with the very long baseline array

S. G. Jorstad, A. P. Marscher, M. L. Lister, A. M. Stirling, T. V. Cawthorne, W. K. Gear, J. L. Gomez, J. A. Stevens, P. S. Smith, J. R. Forster, and E. I. Robson 


\title{
POLARIMETRIC OBSERVATIONS OF 15 ACTIVE GALACTIC NUCLEI AT HIGH FREQUENCIES: JET KINEMATICS FROM BIMONTHLY MONITORING WITH THE VERY LONG BASELINE ARRAY
}

\author{
Svetlana G. Jorstad, ${ }^{1,2}$ Alan P. Marscher, ${ }^{1}$ Matthew L. Lister, ${ }^{3}$ Alastair M. Stirling, ${ }^{4,5}$

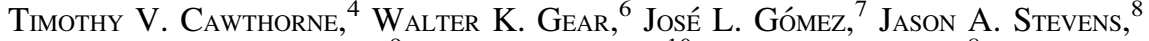 \\ Paul S. Smith, ${ }^{9}$ James R. Forster, $^{10}$ and E. Ian Robson ${ }^{8}$ \\ Received 2005 February 23; accepted 2005 June 27
}

\begin{abstract}
We present total and polarized intensity images of 15 active galactic nuclei obtained with the Very Long Baseline Array at $7 \mathrm{~mm}$ wavelength at 17 epochs from 1998 March to 2001 April. At some epochs the images are accompanied by nearly simultaneous polarization measurements at $3 \mathrm{~mm}, 1.35 / 0.85 \mathrm{~mm}$, and optical wavelengths. Here we analyze the $7 \mathrm{~mm}$ images to define the properties of the jets of two radio galaxies, five BL Lac objects, and eight quasars on angular scales $\gtrsim 0.1$ mas. We determine the apparent velocities of 106 features in the jets. For many of the features we derive Doppler factors using a new method based on a comparison of the timescale of decline in flux density with the light-travel time across the emitting region. This allows us to estimate the Lorentz factors $(\Gamma)$, intrinsic brightness temperatures, and viewing angles of 73 superluminal knots, as well as the opening angle of the jet for each source. The Lorentz factors of the jet flows in the different blazars range from $\Gamma \sim 5$ to 40 with the majority of the quasar components having $\Gamma \sim 16-18$, while the values in the BL Lac objects are more uniformly distributed. The brightest knots in the quasars have the highest apparent speeds, while the more slowly moving components are pronounced in the BL Lac objects. The quasars in our sample have similar opening angles and marginally smaller viewing angles than the BL Lacs. The two radio galaxies have lower Lorentz factors and wider viewing angles than the blazars. Opening angle and Lorentz factor are inversely proportional, as predicted by gasdynamical models. The brightness temperature drops more abruptly with distance from the core in the BL Lac objects than in the quasars and radio galaxies, perhaps owing to stronger magnetic fields in the former resulting in more severe synchrotron losses of the highest energy electrons. In nine sources we detect statistically meaningful deviations from ballistic motion, with the majority of components accelerating with distance from the core. In six sources we identify jet features with characteristics of trailing shocks that form behind the primary strong perturbations in jet simulations. The apparent speeds of these components increase with distance from the core, suggestive of acceleration of the underlying jet.

Key words: BL Lacertae objects: individual (3C 66A, OJ 287, 1803+784, 1823+568, BL Lacertae) galaxies: active - galaxies: individual (3C 111, 3C 120) - galaxies: jets - quasars: individual (0420-014, 0528+134, 3C 273, 3C 279, PKS 1510-089, 3C 345, CTA 102, 3C 454.3)
\end{abstract}

Online material: machine-readable table

\section{INTRODUCTION}

The jets observed emanating from the nuclei of quasars and other active galactic nuclei (AGNs) represent the most energetic long-lived phenomena in the universe. Although jet imaging is no longer the sole prerogative of radio astronomy, the exquisite resolution of very long baseline interferometry (VLBI) at radio wavelengths remains an unreached goal for submillimeter and

\footnotetext{
${ }^{1}$ Institute for Astrophysical Research, Boston University, 725 Commonwealth Avenue, Boston, MA 02215-1401; jorstad@bu.edu, marscher@bu.edu.

${ }^{2}$ Sobolev Astronomical Institute, St. Petersburg State University, Universitetskij Pr. 28, 198504 St. Petersburg, Russia.

3 Department of Physics, Purdue University, 525 Northwestern Avenue, West Lafayette, IN 47907-2036; mlister@physics.purdue.edu.

${ }^{4}$ Center for Astrophysics, University of Central Lancashire, Preston PR1 2HE, UK; tvcawthorne@uclan.ac.uk.

${ }^{5}$ Current address: University of Manchester, Jodrell Bank Observatory, Macclesfield, Cheshire SK11 9DL, UK; ams@jb.man.ac.uk.

${ }^{6}$ School of Physics and Astronomy, Cardiff University, 5 The Parade, Cardiff CF2 3 YB, Wales, UK; walter.gear@astro.cf.ac.uk.

7 Insituto de Astrofísica de Andalucía (CSIC), Apartado 3004, Granada 18080, Spain; jlgomez@iaa.es.

8 Astronomy Technology Centre, Royal Observatory, Blackford Hill, Edinburgh EH93HJ, UK; jas@roe.ac.uk, eir@roe.ac.uk.

9 Steward Observatory, University of Arizona, 933 North Cherry Avenue, Tucson, AZ 85721; psmith@as.arizona.edu.

${ }^{10}$ Hat Creek Observatory, University of California, Berkeley, 42231 Bidwell Road, Hat Creek, CA 96040; rforster@astro.berkeley.edu.
}

shorter wavelengths. It is thought that accretion onto a black hole drives the jet outward via magnetic forces (e.g., Meier et al. 2001). Currently, the most direct way to provide observational evidence for such a model is to study the linear polarization of jets at different frequencies (from radio to optical) along with changes in the innermost jet structure. The most promising objects in such investigations are blazars, flat-spectrum radio-loud quasars, and BL Lac objects, characterized by high optical polarization up to 46\% (Mead et al. 1990; Impey \& Tapia 1990), pronounced and rapid variability offlux, and one-sided jets with knots that move at superluminal apparent velocities, as fast as $\gtrsim 30$ c (Jorstad et al. 2001b; Kellermann et al. 2004). According to Wills et al. (1992), there is a highly significant correlation between optical polarization and dominance of a compact radio core-jet structure, which suggests beaming of the optical flux along with the radio emission. Comparison of radio-loud quasars with low and high optical polarization shows a higher fractional polarization of the radio core for the latter (Lister \& Smith 2000), which implies a cospatial origin of the emission at these wavelengths. In support of this, the optical polarization in blazars is affected by the formation and emergence of new VLBI knots (Gabuzda \& Sitko 1994; Gabuzda et al. 1996). These results indicate that simultaneous multifrequency polarization monitoring, together with high-resolution polarimetric imaging of the radio jets, provides a unique tool for identifying the location of the regions responsible 
TABLE 1

Sample and Parameters of Maps Shown in Figs. 1-15

\begin{tabular}{|c|c|c|c|c|c|c|c|c|}
\hline Source & Type & $z$ & $\begin{array}{l}\text { Beam } \\
\text { (mas) }\end{array}$ & $\begin{array}{l}\text { P.A. } \\
\text { (deg) }\end{array}$ & $\begin{array}{c}I_{\text {peak }} \\
\left(\mathrm{mJy} \mathrm{beam}^{-1}\right)\end{array}$ & $\begin{array}{c}I_{\text {peak }}^{p} \\
\left(\mathrm{mJy} \mathrm{beam}^{-1}\right)\end{array}$ & $\begin{array}{c}I_{\min } \\
\left(\mathrm{mJy} \mathrm{beam}^{-1}\right)\end{array}$ & $\begin{array}{c}I_{\min }^{p} \\
\left(\mathrm{mJy} \mathrm{beam}^{-1}\right)\end{array}$ \\
\hline 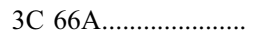 & BL & 0.444 & $0.33 \times 0.20$ & -15 & 745 & 60 & 1.9 & 5 \\
\hline 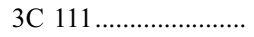 & RG & 0.048 & $0.30 \times 0.20$ & -10 & 2960 & 14 & 6.0 & 3 \\
\hline 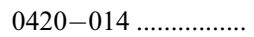 & Q & 0.915 & $0.38 \times 0.15$ & -8 & 6265 & 211 & 12.5 & 11 \\
\hline 3C $120 \ldots \ldots \ldots \ldots \ldots \ldots$ & RG & 0.033 & $0.40 \times 0.15$ & -6 & 1520 & 36 & 7.6 & 3 \\
\hline $0528+134 \ldots \ldots \ldots \ldots \ldots$ & Q & 2.06 & $0.30 \times 0.15$ & -7 & 3930 & 131 & 18.6 & 6 \\
\hline 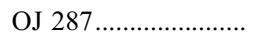 & $\mathrm{BL}$ & 0.306 & $0.30 \times 0.14$ & -7 & 2330 & 96 & 8.1 & 5 \\
\hline 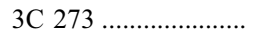 & Q & 0.158 & $0.38 \times 0.14$ & -6 & 12800 & 943 & 44.8 & 45 \\
\hline 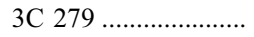 & Q & 0.538 & $0.38 \times 0.14$ & -6 & 17240 & 1240 & 26.0 & 62 \\
\hline $1510-089$................... & Q & 0.361 & $0.40 \times 0.15$ & -6 & 2580 & 147 & 9.0 & 7 \\
\hline 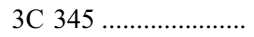 & Q & 0.595 & $0.22 \times 0.16$ & -9 & 5055 & 358 & 17.7 & 21 \\
\hline $1803+784 \ldots \ldots \ldots \ldots \ldots$ & $\mathrm{BL}$ & 0.68 & $0.16 \times 0.15$ & 25 & 1490 & 94 & 5.2 & 5 \\
\hline $1823+568 \ldots \ldots \ldots \ldots \ldots$ & BL & 0.664 & $0.19 \times 0.16$ & -10 & 1580 & 131 & 7.9 & 5 \\
\hline BL Lac ........................ & $\mathrm{BL}$ & 0.069 & $0.28 \times 0.15$ & -3 & 3810 & 184 & 9.5 & 9 \\
\hline CTA $102 \ldots \ldots \ldots \ldots \ldots . . . . . . .$. & Q & 1.037 & $0.25 \times 0.15$ & -3 & 4180 & 124 & 10.4 & 6 \\
\hline 3C $454.3 \ldots \ldots \ldots \ldots \ldots \ldots$ & $\mathrm{Q}$ & 0.859 & $0.31 \times 0.15$ & -5 & 5070 & 358 & 17.7 & 18 \\
\hline
\end{tabular}

for variability at different wavelengths and for relating the magnetic field geometry to the structure of the jet.

We have obtained total and polarized intensity images of 15 AGNs with the Very Long Baseline Array (VLBA) at $7 \mathrm{~mm}$ $(43 \mathrm{GHz})$ at 17 epochs over 3 years. The VLBA observations are accompanied at many epochs by nearly simultaneous (within 2 weeks) measurements of polarization at $1.35 / 0.85 \mathrm{~mm}(230 /$ $350 \mathrm{GHz}$ ) and at optical wavelengths. In the second half of the program, simultaneous polarization observations at $3 \mathrm{~mm}$ were performed at several epochs. The main goals of the project are to relate emission regions at high frequencies to the parsec-scale jet structure and to investigate the strength, direction, and variability of the magnetic field close to the central engine. These can be achieved only after detailed study of the jet kinematics. This paper is devoted to an analysis of the jet structure and its variability associated with ejection and propagation of disturbances down the jet, which appear on radio maps as knots of enhanced brightness. The paper is the first in a series based on the entire data set collected during the project. Some results on individual sources have been published already by Marscher et al. (2002; 3C 120), Stirling et al. (2003; BL Lac), and Jorstad et al. (2004; 3 C 279). Other papers will include (1) comparison of the polarization parameters at different frequencies with the jet structure and disturbances in the jet; (2) analysis of the available radio, submillimeter, optical, and X-ray light curves to relate the flux variability to activity in the jet; (3) results regarding stability of the VLBI core position based on phase-referencing observations obtained for five sources in the sample; and (4) structure of the core and intraday total and polarized intensity variability in the parsec-scale jets.

Although much progress has been made in studying the kinematics of jets since the VLBA started to operate fully in 1995 (Pearson et al. 1998; Kellermann et al. 1998, 2004; Homan et al. 2001; Jorstad et al. 2001b; Gómez et al. 2001), our program is unique regarding the number of sources observed in such detail over a rather long period of time. This allows us to separate the fast and slow, and the ballistic and curved motion in the jet flow, define physical parameters for both individual jet features and entire parsecscale jets, and compare the results across different classes of AGNs.

\section{SAMPLE SELECTION}

Our program is designed for comparison of the linear polarization at high frequencies (millimeter, submillimeter, and optical wavelengths) with the parsec-scale jet structure of AGNs. This has defined the main criteria for selecting the sample and frequency of our VLBA observations:

1. The sources should be bright, $\geq 0.5 \mathrm{Jy}$, and polarized, $\geq 3 \%$, at submillimeter wavelengths.

2. The size of the sample and brightness of sources should allow us to perform VLBA observations at a single epoch during $24 \mathrm{hr}$ with sufficient $(u, v)$ coverage to produce total and polarized intensity images at $43 \mathrm{GHz}$ with high dynamic range.

3 . The sample should contain sources with resolved radio structure from different subclasses of AGNs for which variability in the jet flow can be expected on timescales of months.

4. The sources should be convenient for monitoring in the Northern Hemisphere, and their coordinates should cover the whole range of right ascensions.

Following these constraints, we have formed a sample of 15 AGNs that consists of eight quasars, five BL Lac objects, and two radio galaxies (3C 120 of Fanaroff-Riley type 1 and 3C 111 of type 2) after obtaining information on the linear polarization at submillimeter wavelengths from Nartallo et al. (1998). The sources are listed in Table 1.

\section{OBSERVATIONS AND DATA REDUCTION}

We have observed the objects in our sample in four different regions of the electromagnetic spectrum: at $43 \mathrm{GHz}(7 \mathrm{~mm})$ with the VLBA, at $350 / 230 \mathrm{GHz}(0.85 / 1.3 \mathrm{~mm})$ with the James Clerk Maxwell Telescope (JCMT; Mauna Kea, Hawaii) using SCUBA (Holland et al. 1999) and its polarimeter (Greaves et al. 2003), at the Steward Observatory $1.5 \mathrm{~m}$ telescope (Mount Lemmon, Arizona) with the Two-Holer Polarimeter/Photometer (Sitko et al. 1985) over an effective wavelength range of $\sim 6000-7000 \AA$, and at $86 \mathrm{GHz}(3 \mathrm{~mm})$ with the Berkeley-Illinois-Maryland Association (BIMA) array (Hat Creek, California). The observations were performed from 1998 March to 2001 April. The VLBA monitoring was carried out roughly bimonthly (17 epochs). For the JCMT and optical observations the number of epochs depends on the source, with the maximum being 11 and seven epochs and the minimum five and three epochs, respectively, except for the BL Lac object 1803+784, which was not observed in the optical region owing to an inaccessibly high declination. The majority of the JCMT polarization observations were accompanied by total flux measurements. Differential $V$-band photometry $(R$-band 
TABLE 2

Log of VLBA OBSERVATIONS

\begin{tabular}{|c|c|c|c|c|c|c|c|c|}
\hline Epoch & Antennas & $\begin{array}{l}\text { Beam } \\
\text { (mas) }\end{array}$ & $\begin{array}{l}\text { P.A. } \\
\text { (deg) }\end{array}$ & $f_{\text {amp }}$ & $\begin{array}{c}\sigma(\text { EVPA }) \\
\quad(\operatorname{deg})\end{array}$ & BIMA Obs. & JCMT Obs. & Optical Obs. \\
\hline 1998 Mar 25 (1998.23) ........ & All & $0.29 \times 0.14$ & -5 & $1.26 \pm 0.10$ & 7 & No & Yes & No \\
\hline 1998 May $30(1998.41) \ldots \ldots . .$. & All & $0.29 \times 0.14$ & -7 & $1.24 \pm 0.08$ & 5 & No & Yes & No \\
\hline 1998 Aug 1 (1998.58) .......... & All & $0.29 \times 0.15$ & -11 & $1.30 \pm 0.07$ & 5 & No & Yes & No \\
\hline 1998 Oct 5 (1998.76) ........... & All & $0.29 \times 0.14$ & -4 & $1.48 \pm 0.20$ & 8 & No & Yes & No \\
\hline 1998 Dec 10 (1998.94)......... & All & $0.29 \times 0.13$ & -9 & $1.57 \pm 0.11$ & 8 & No & Yes & No \\
\hline 1999 Feb $11(1999.12) \ldots \ldots \ldots .$. & All & $0.31 \times 0.14$ & -12 & $1.48 \pm 0.15$ & 8 & No & Yes & Yes \\
\hline 1999 Apr 29 (1999.33)......... & All, bad weather & $0.27 \times 0.14$ & -6 & $1.27 \pm 0.11$ & 8 & No & Yes & Yes \\
\hline 1999 Jul 18 (1999.55) .......... & All & $0.31 \times 0.15$ & -12 & $0.7 \pm 0.2$ & 10 & No & No & No \\
\hline 1999 Oct $6(1999.76) \ldots \ldots \ldots \ldots . .$. & All & $0.31 \times 0.16$ & -3 & $1.38 \pm 0.10$ & 6 & No & Yes & No \\
\hline 1999 Dec 5 (1999.94).......... & All besides Saint Croix & $0.38 \times 0.15$ & -20 & $1.38 \pm 0.12$ & 8 & No & Yes & Yes \\
\hline 2000 Jan $24(2000.07) \ldots \ldots \ldots . .$. & All & $0.27 \times 0.15$ & -5 & $1.25 \pm 0.08$ & 6 & No & No & No \\
\hline 2000 Apr $5(2000.26) \ldots \ldots \ldots \ldots$ & All & $0.28 \times 0.15$ & -8 & $1.24 \pm 0.08$ & 6 & Yes & Yes & Yes \\
\hline 2000 Jul 17 (2000.54) .......... & All & $0.28 \times 0.14$ & -7 & $1.13 \pm 0.10$ & 8 & No & No & No \\
\hline 2000 Oct $1(2000.75) \ldots \ldots \ldots \ldots$ & All besides Brewster & $0.32 \times 0.14$ & -8 & $1.23 \pm 0.14$ & 8 & No & Yes & No \\
\hline 2000 Dec $11(2000.95) \ldots \ldots \ldots .$. & All besides Mauna Kea & $0.27 \times 0.14$ & -2 & $1.40 \pm 0.20$ & 12 & Yes & Yes & Yes \\
\hline 2001 Jan $28(2001.08) \ldots \ldots \ldots .$. & All, bad weather & $0.29 \times 0.14$ & -1 & $1.32 \pm 0.10$ & 15 & Yes & Yes & No \\
\hline 2001 Apr $14(2001.28) \ldots \ldots \ldots . .$. & All & $0.28 \times 0.14$ & -8 & $1.19 \pm 0.08$ & 5 & Yes & No & No \\
\hline
\end{tabular}

photometry in the case of 3C 273) was carried out when conditions were photometric. The BIMA polarization observations started in 2000 April and for many sources in the sample were performed simultaneously with the VLBA observations at three or four epochs. However, each of these epochs usually was accompanied by a series of BIMA observations separated by several days so that for some sources polarization measurements at $3 \mathrm{~mm}$ were obtained at 20 epochs. The polarization data at $3 \mathrm{~mm}$ include all four Stokes parameters. In this paper the optical, JCMT, and BIMA polarization results are shown only graphically to illustrate the data collected. Detailed descriptions of the data reduction and tables of measurements will be presented in a later paper.

\subsection{Radio Observations}

The $43 \mathrm{GHz}$ observations were carried out with the VLBA recording system using eight $8 \mathrm{MHz}$ wide channels, each in right and left circular polarization, with 15-20 scans of 3-5 minute duration for each object. All 10 antennas were used for each source except at epochs affected by weather or receiver failure. Table 2 lists the antennas operated at each epoch and typical parameters of the synthesized beam (those of the OJ 287 observations) for uniform weighting (used for imaging all sources) to illustrate the consistency of the $(u, v)$ coverage over epochs. Initial correlation was carried out at the National Radio Astronomy Observatory (NRAO) Array Operations Center in Socorro, New Mexico. Subsequent calibration was performed with the Astronomical Image Processing System (AIPS) software supplied by NRAO, while images were made with the Caltech software DIFMAP (Shepherd 1997). The calibration included application of the nominal antenna-based gain curves and system temperatures, as well as correction for sky opacity, followed by iterative imaging plus phase and amplitude self-calibration. For each epoch we calculated the total flux density in the images of sources that are known to have very weak emission outside the angular size range of the VLBA images (0420-014, 0528+134, OJ 287, and BL Lac). These values were compared with total flux densities obtained by interpolating in time the measurements of the monitoring program at $37 \mathrm{GHz}$ at the Metsähovi Research Station in Finland (Teräsranta et al. 2004). The comparison produced the flux-density correction factors, $f_{\text {amp }}$, given in Table 2 . The factors were applied for the final adjustment of the flux-density scale in the images. We have constructed light curves of the VLBI core for each source to confirm the absence of correlation between their behaviors, indicating no evidence for residual amplitude calibration errors. The light curves of the 15 sources peak at nine different epochs, and the correlation coefficients are spread between -0.4 and +0.3 , consistent with no systematic calibration errors in the flux density scaling.

We performed a cross-hand fringe fit using a scan of 3C 279 averaged over all baselines (the data were corrected for parallactic angle rotation of phases in advance). The resulting right-left (R-L) phase and rate delay corrections were processed with the AIPS task POLSN and applied to the full data set. After preliminary images were produced, the images were used in AIPS task CALIB to self-calibrate the phases of all sources; this included the removal of residual R-L phase differences owing to calibration errors. The instrumental polarization "D-terms" were determined via the method of Roberts et al. (1994) and Leppänen et al. (1995). A final set of D-terms at each epoch was obtained by averaging the solutions for those sources in the sample for which there is the best agreement between the D-terms (usually, 3C 111, OJ 287, 3C 345, and BL Lac). The electric vector position angle (EVPA) calibration was obtained by different methods: comparison between the Very Large Array (VLA) and VLBA integrated EVPAs at quasi-simultaneous epochs, the D-term method (see below), and using EVPA-stable features in the images of the jets of 3C 279, OJ 287, and CTA 102. Over the period from 1998 March to 1999 February we used results of the VLA observations performed for $0420-014$, 3C 120, OJ 287, 3C 279, BL Lac, and 3C 454.3 by Gómez et al. (2002). Later we obtained polarization measurements for $0420-014,0528+134$, OJ 287, and $1803+784$ at two epochs, 2000 April and July, with the VLA C and CnD configuration, respectively. At epochs when VLA data were not available we used the D-term method, which is based on the assumption that the instrumental polarization parameters change slowly with time (Leppänen et al. 1995). A detailed description of the method is given in Gómez et al. (2002). In addition, we later checked our calibration for these epochs using the NRAO database ${ }^{11}$ for sources

\footnotetext{
${ }^{11}$ See http://www.vla.nrao.edu/astro/calib/polar.
} 

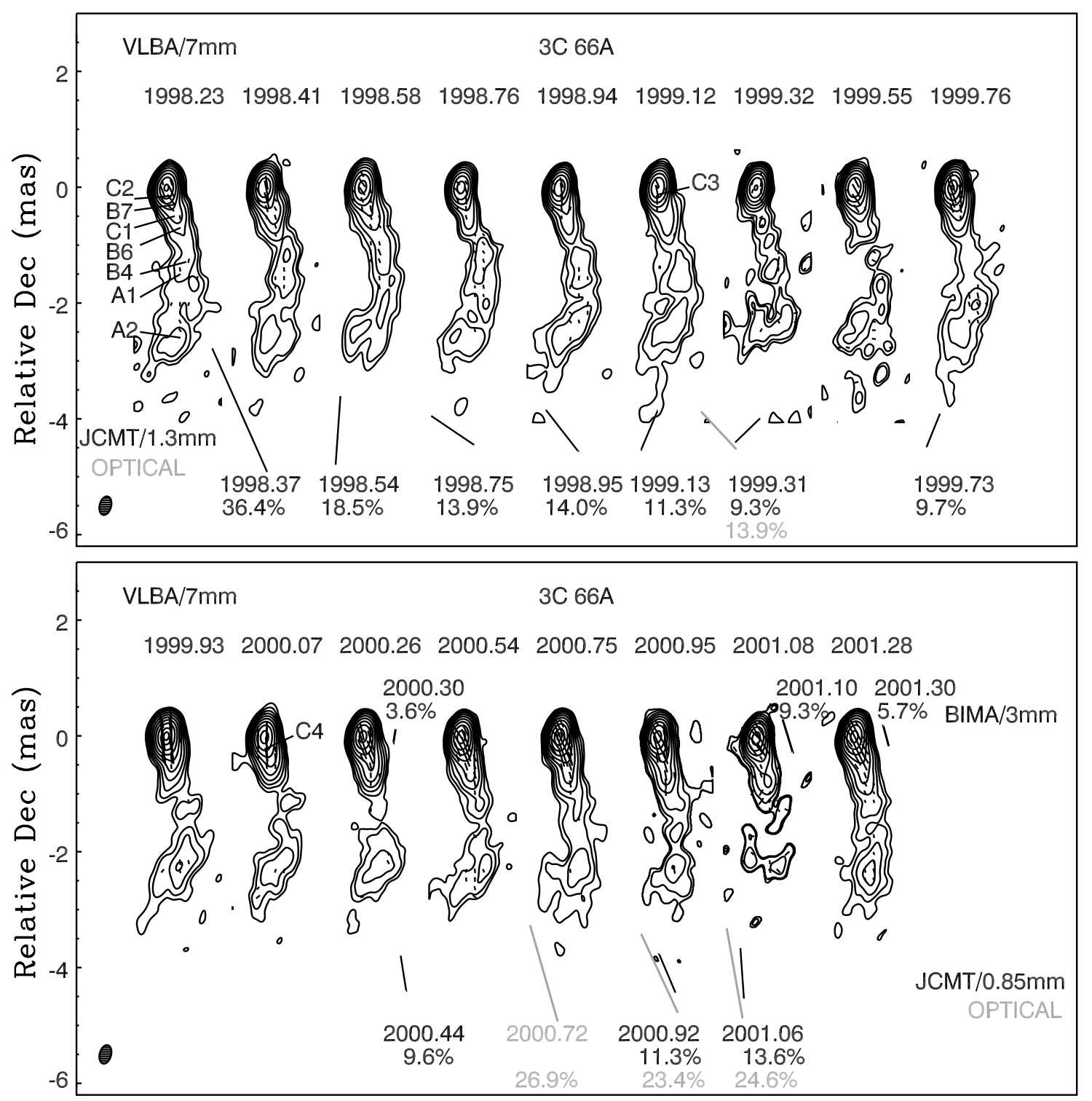

FIG. 1.-Total intensity images of $3 \mathrm{C} 66 \mathrm{~A}$ at $43 \mathrm{GHz}$. North is toward the top, and east is toward the left. Line segments within each image indicate direction of electric vectors, with length proportional to the polarized intensity at $7 \mathrm{~mm}$. Line segments outside the images give the direction of polarization at $3 \mathrm{~mm}$ (dark gray), $0.85 / 1.3 \mathrm{~mm}$ (black), and optical wavelengths (light gray) with length reflecting the percent of polarization. The percent of polarization and the epoch of observation are printed near the corresponding line segments. The darkness of the print indicates the wavelength of observation. If epochs of the JCMT and optical observations coincide, only the JCMT epoch is indicated. Parameters of the maps are listed in Table 1. The resolution beam is shown by the cross-hatched ellipse in the lower left corner. The designation of knots follows Table 5.

0420-014, 0528+134, OJ287, and BL Lac when the NRAO data were available at epochs close to the VLBA dates. The results are completely consistent with our calibration. The EVPAstable feature in 3C 279 is the well-known superluminal component C4 (e.g., Homan et al. 2003), which underwent a change in EVPA in the beginning of 1998 (Jorstad et al. 2004) but maintained this EVPA until the end of our program. The EVPA-stable features in OJ 287 and CTA 102 are quasi-stationary components at $\sim 1$ and $\sim 2$ mas from the core, respectively. However, these both became very weak in the second half of 2000 in the $43 \mathrm{GHz}$ images. The final EVPA calibration is a result of the best agreement among the different methods. The accuracy of the calibrated EVPA measurements, $\sigma$ (EVPA), is indicated in Table 2.

We have combined the images obtained for every source in a sequence of total intensity maps convolved with the same beam, corresponding to the average beam during the epochs when all 10 antennas were in operation. The contours are in terms of the global peak of the maximum total intensity observed over all epochs. The sequences are presented in Figures 1-15, which also show the JCMT, BIMA, and optical polarization measurements. All images are oriented with north toward the top and east toward the left. The scale in milliarcseconds is indicated along one of the axes, while the epoch of each image is given along the other axis. The peaks of the total $\left(I_{\text {peak }}\right)$ and polarized $\left(I_{\text {peak }}^{p}\right)$ intensity, parameters of the average beam, the total intensity of the lowest contour $\left(I_{\min }\right)$, and the lowest level of the polarized intensity $\left(I_{\min }^{p}\right)$ of the combined maps are indicated in Table 1. The polarization at $7 \mathrm{~mm}$ is shown inside the total intensity images by line segments, which are plotted if the local polarized intensity exceeds $I_{\min }^{p}$. The segments are oriented in the local direction of the polarization, and their length is proportional to the local polarized intensity, with the maximum length corresponding to the global peak of the polarized intensity over all epochs, $I_{\text {peak }}^{p}$. Table 2 marks epochs when the BIMA, JCMT, 


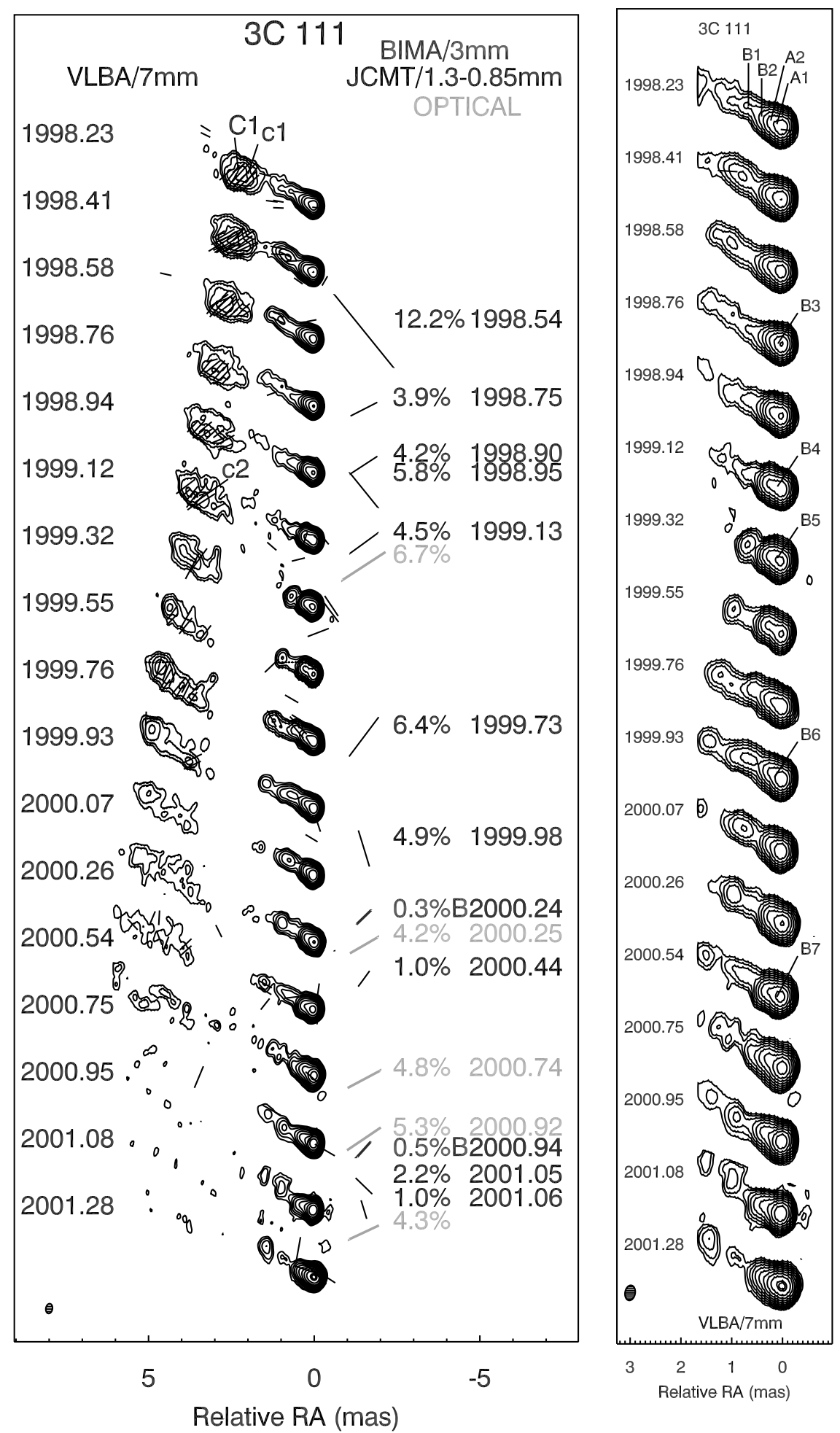

FIG. 2.-Left: Total intensity images of $3 \mathrm{C} 111$ at $43 \mathrm{GHz}$ along with polarization measurements at 7, 3, and 1.3/0.85 mm and at optical wavelengths. The epochs of BIMA observations are marked by the letter B. See Fig. 1 for details. Right: Total intensity images of the inner jet of 3C 111 at $43 \mathrm{GHz}$. 

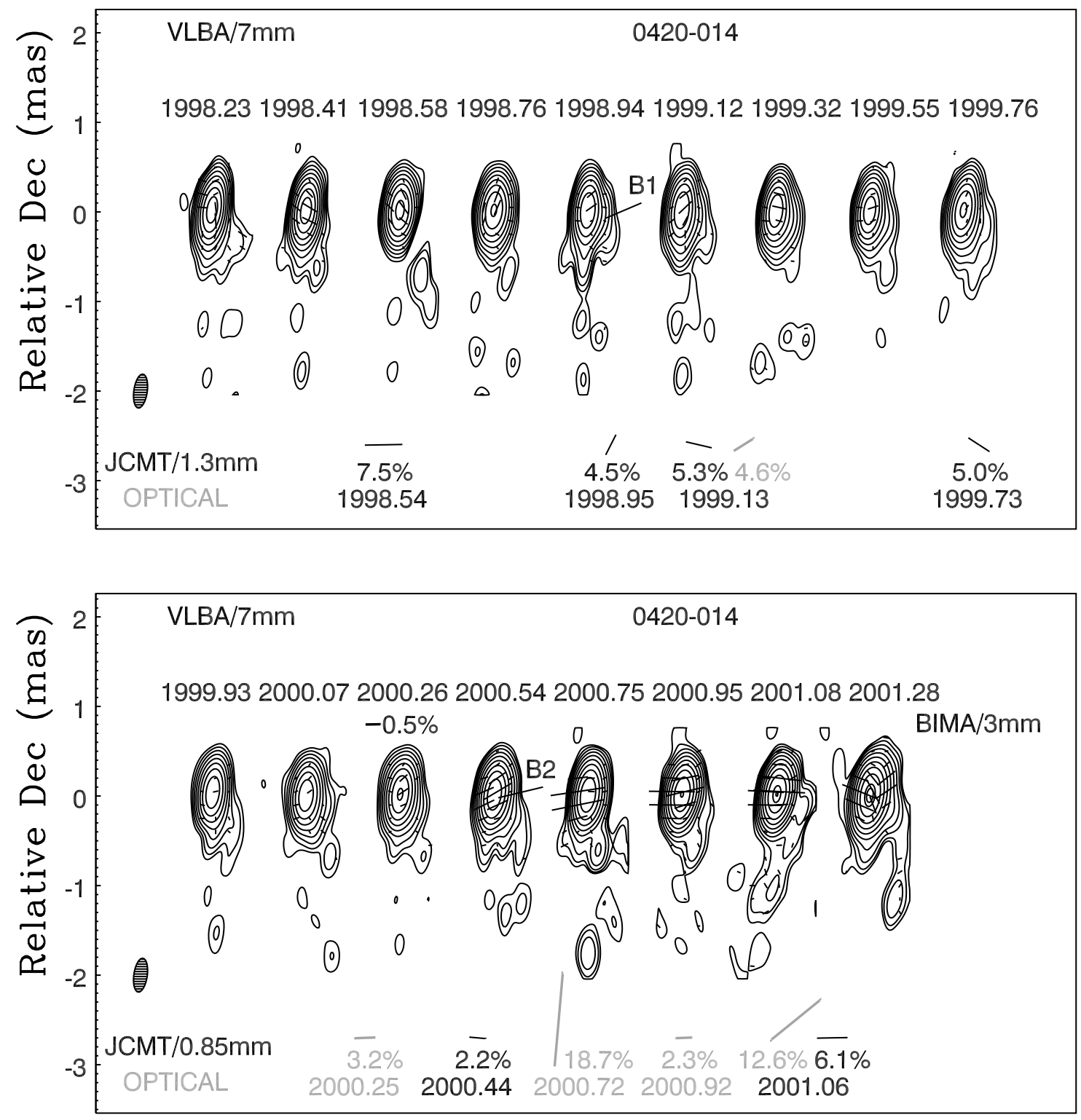

Fig. 3.-Total intensity images of $0420-014$ at $43 \mathrm{GHz}$ along with polarization measurements at 7, 3, and 1.3/0.85 mm and at optical wavelengths. The epochs of BIMA observations coincide with the epochs of corresponding VLBA images. See the legend for Fig. 1. 

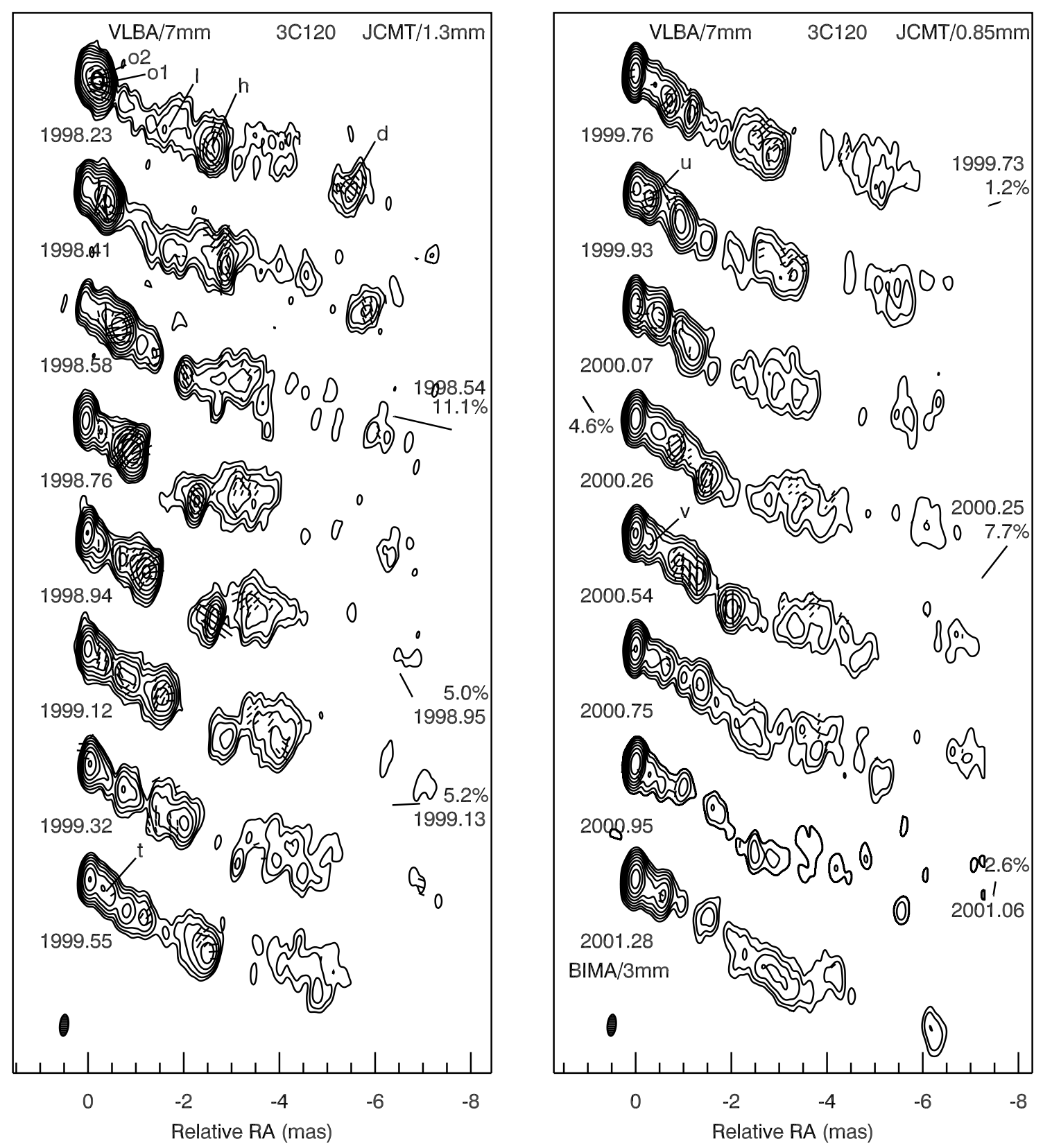

FIG. 4.-Total intensity images of $3 \mathrm{C} 120$ at $43 \mathrm{GHz}$ along with polarization measurements at 7, 3, and 1.3/0.85 mm. Measurements of the optical polarization are consistent with Galactic interstellar polarization for this sight line and are not included. The epochs of BIMA observations coincide with the epochs of corresponding VLBA images. See the legend for Fig. 1. 

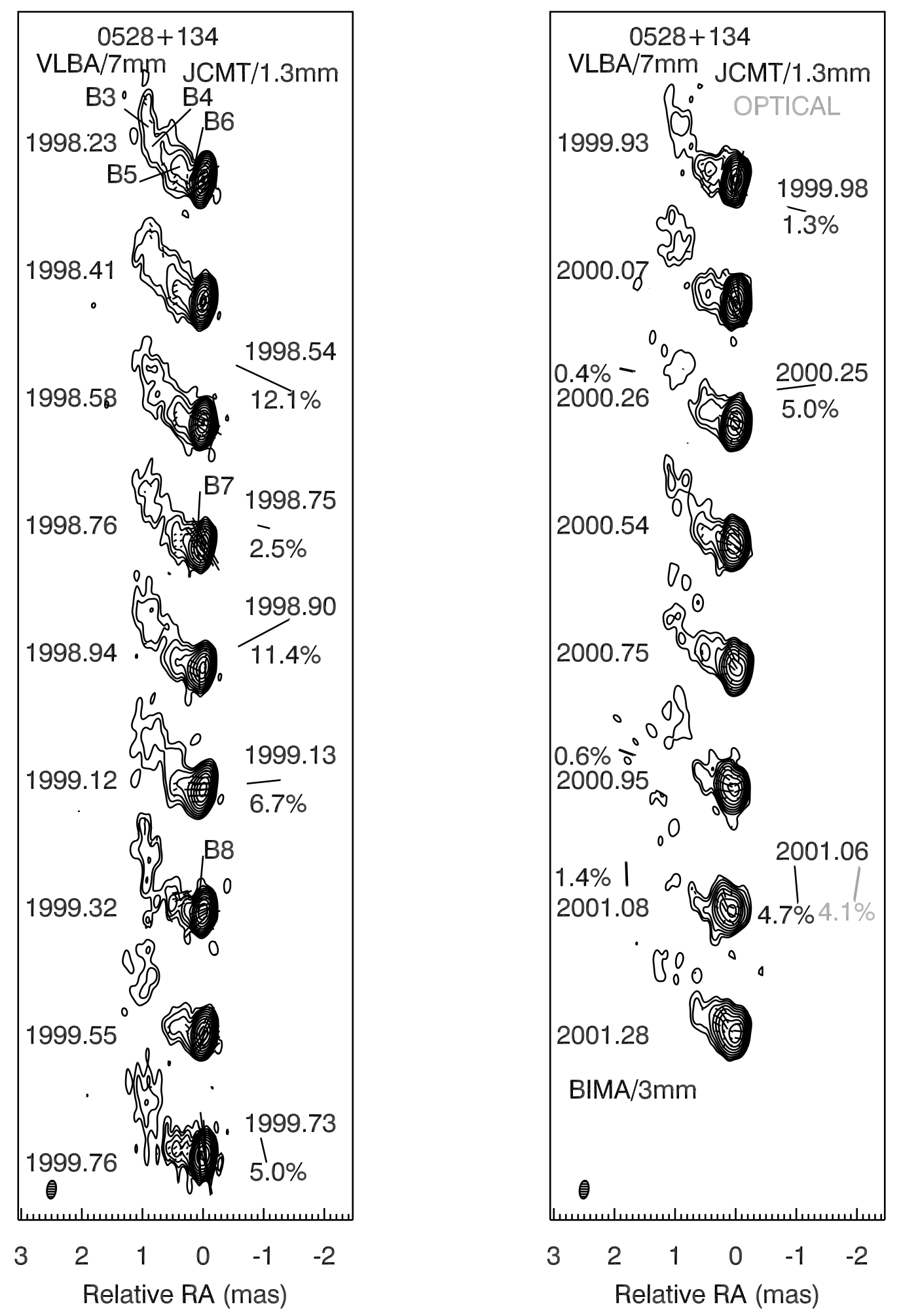

FIG. 5.-Total intensity images of $0528+134$ at $43 \mathrm{GHz}$ along with polarization measurements at 7,3 , and $1.3 / 0.85 \mathrm{~mm}$ and at optical wavelengths. The epochs of BIMA observations coincide with the epochs of corresponding VLBA images. See the legend for Fig. 1. 

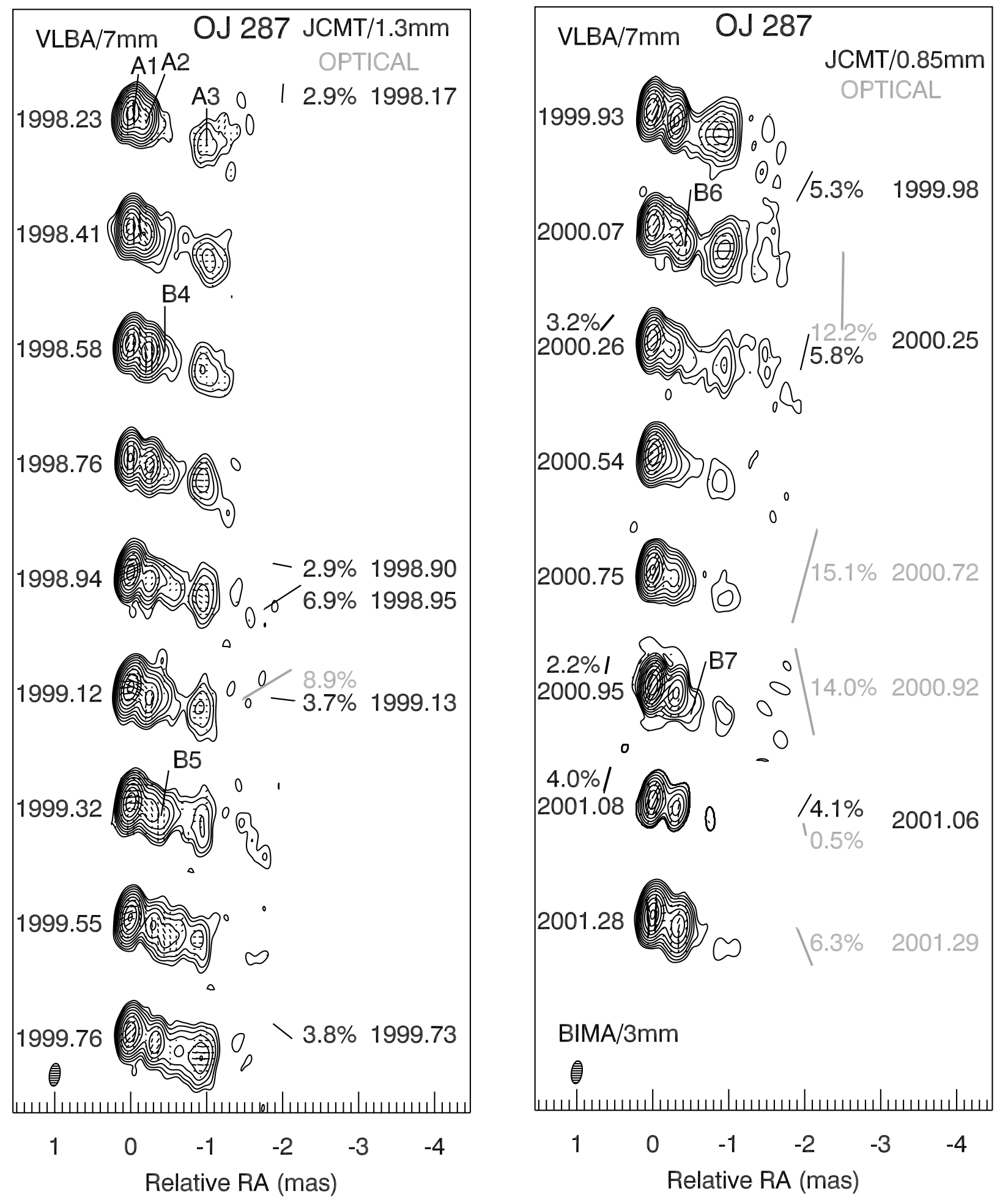

FIG. 6.-Total intensity images of OJ 287 at $43 \mathrm{GHz}$ along with polarization measurements at 7,3, and 1.3/0.85 mm and at optical wavelengths. The epochs of BIMA observations coincide with the epochs of corresponding VLBA images. See the legend for Fig. 1. 

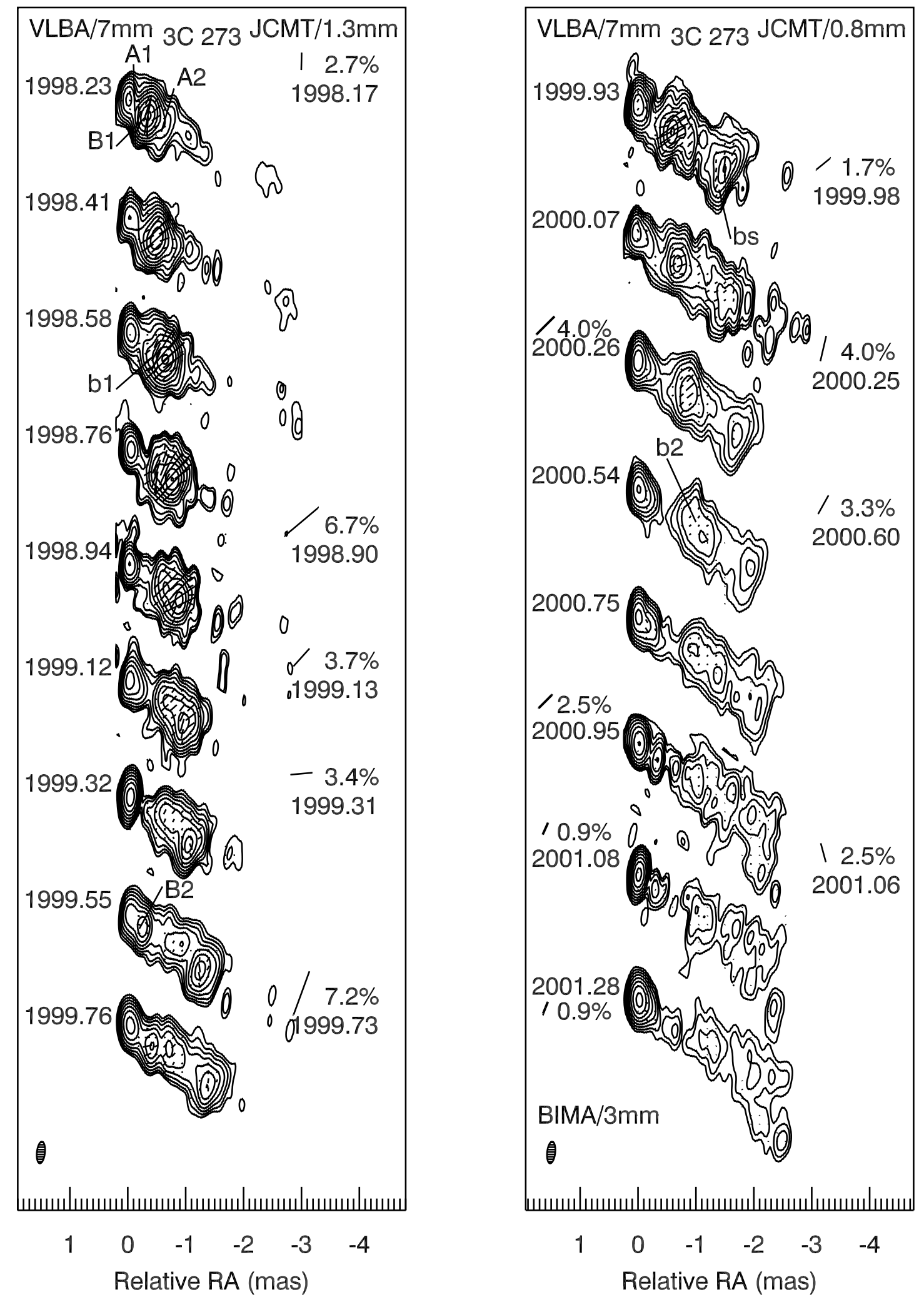

FIG. 7.-Total intensity images of 3C 273 at $43 \mathrm{GHz}$ along with polarization measurements at 7, 3, and 1.3/0.85 mm. The epochs of BIMA observations coincide with the epochs of corresponding VLBA images. See the legend for Fig. 1. 

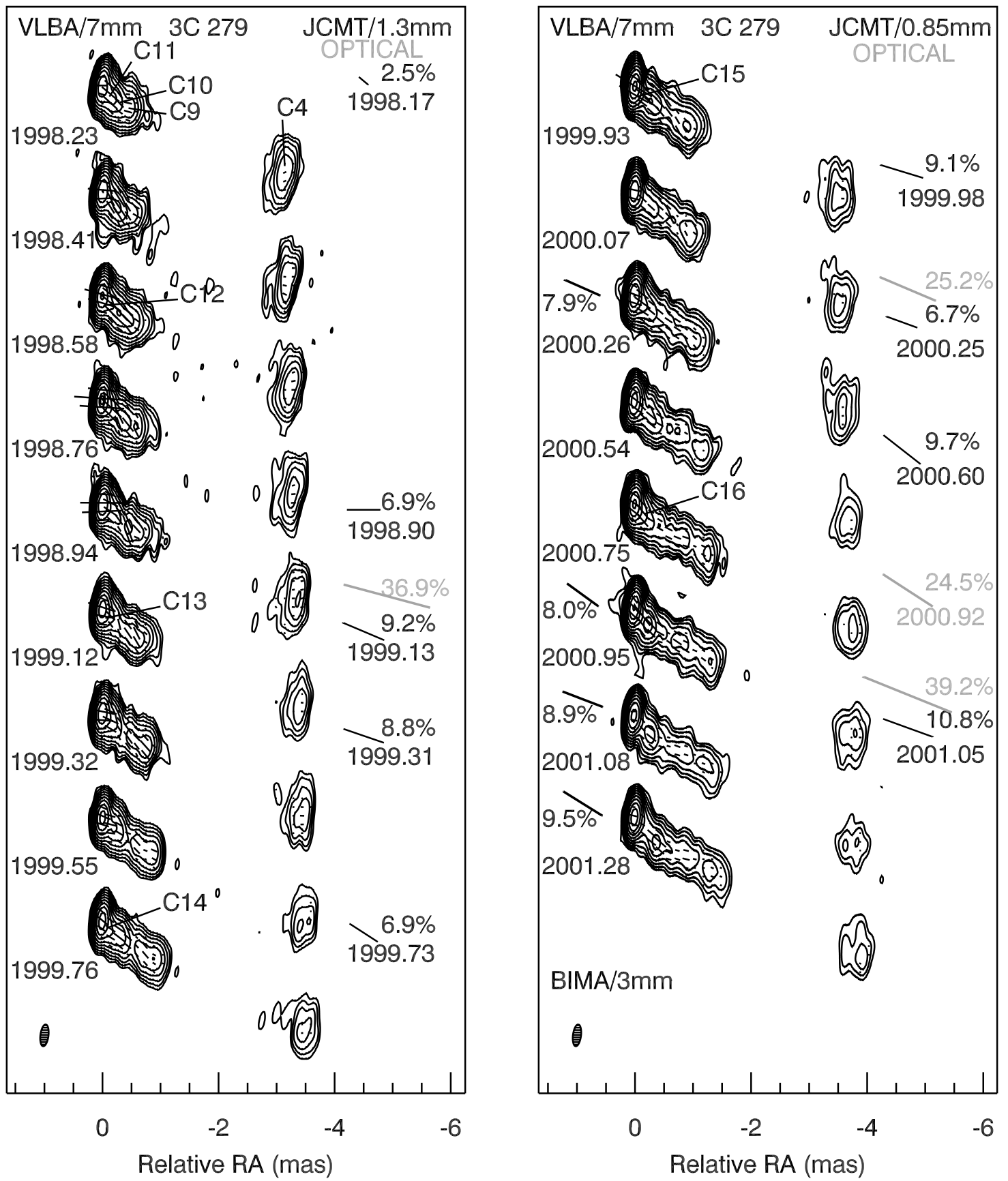

FIG. 8.-Total intensity images of 3C 279 at $43 \mathrm{GHz}$ along with polarization measurements at 7, 3, and 1.3/0.85 mm and at optical wavelengths. The epochs of BIMA observations coincide with the epochs of corresponding VLBA images. See the legend for Fig. 1. 


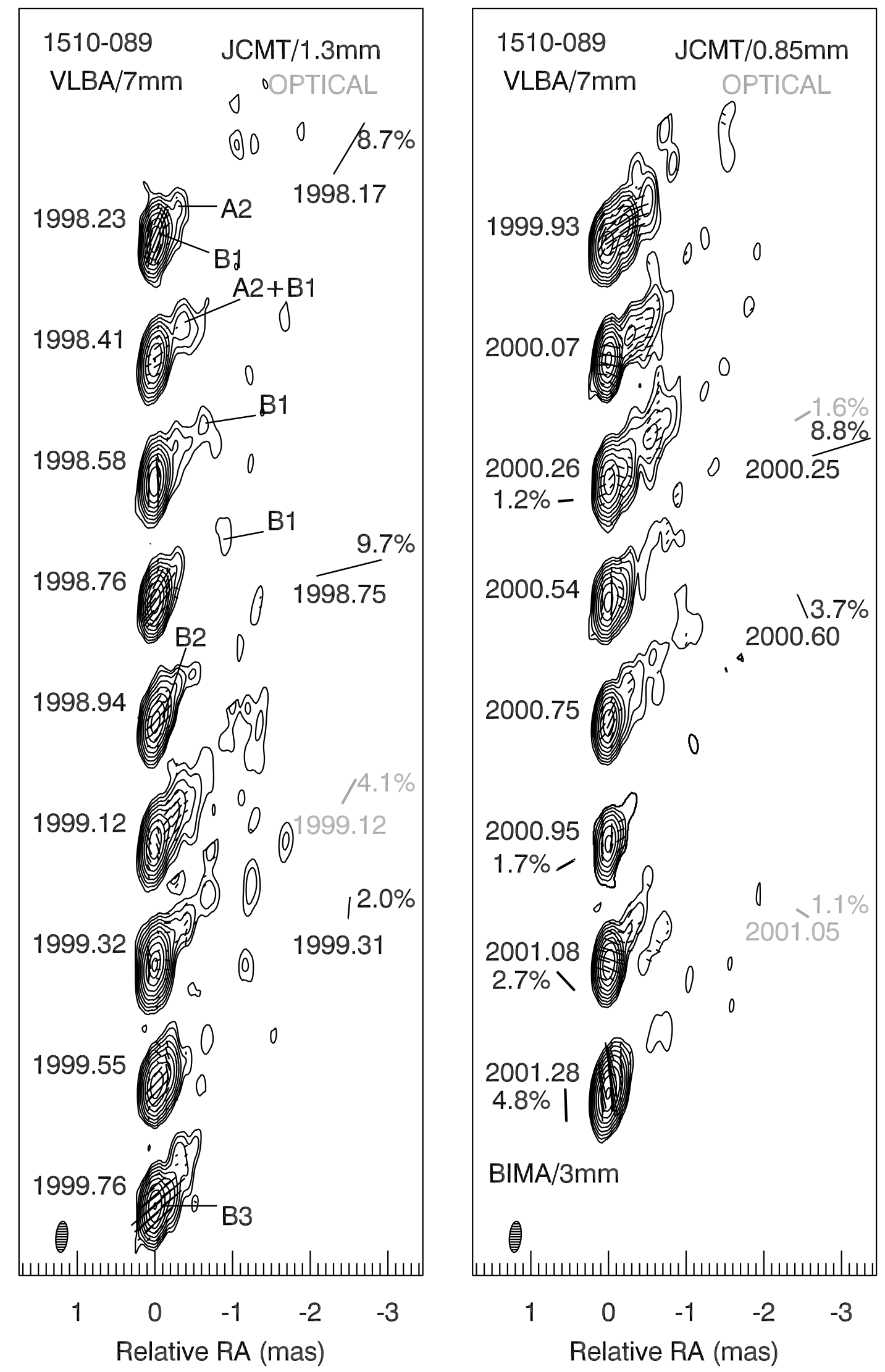

FIG. 9.- Total intensity images of PKS $1510-089$ at $43 \mathrm{GHz}$ along with polarization measurements at 7, 3, and 1.3/0.85 mm and at optical wavelengths. The epochs of BIMA observations coincide with the epochs of corresponding VLBA images. See the legend for Fig. 1. 


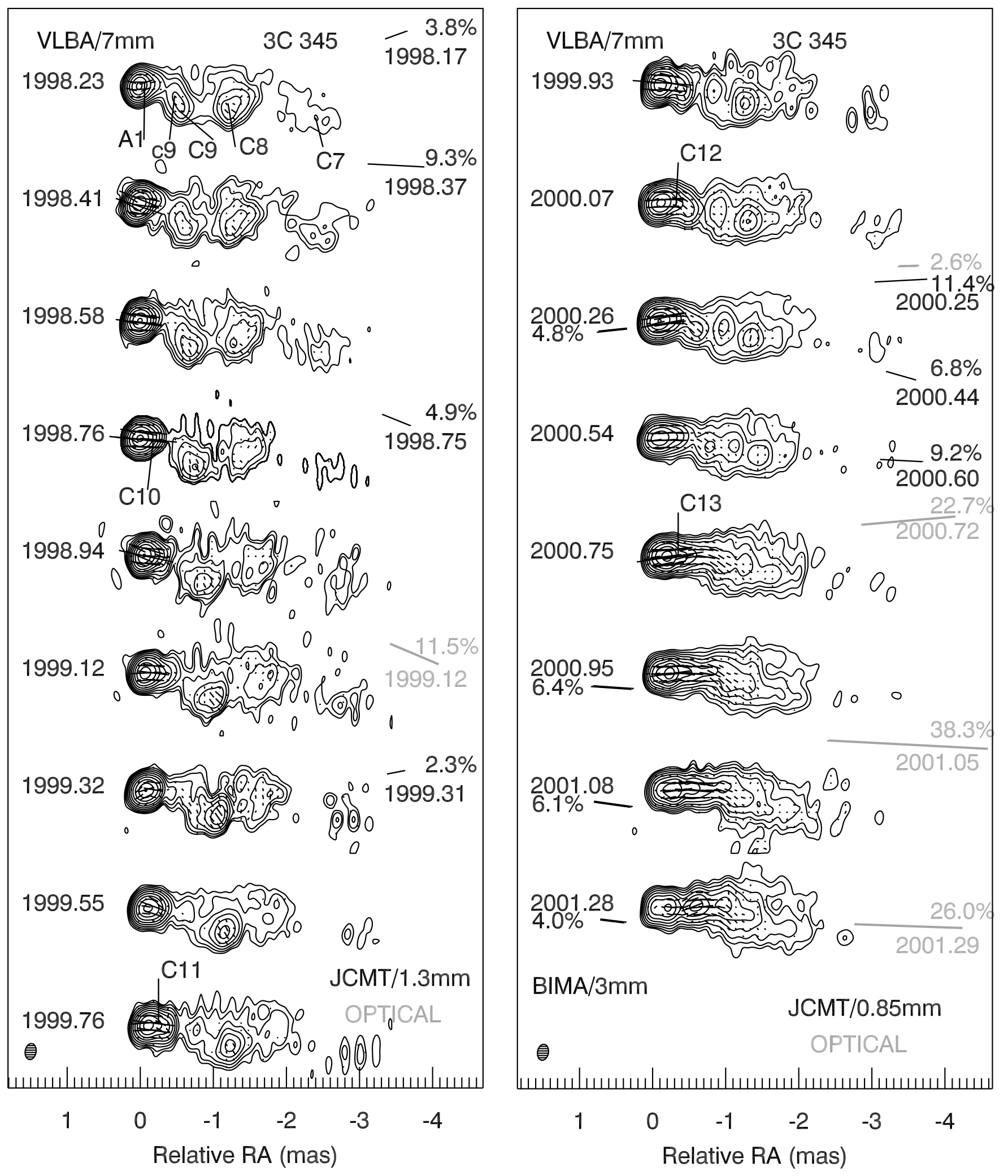

FIG. 10.-Total intensity images of $3 \mathrm{C} 345$ at $43 \mathrm{GHz}$ along with polarization measurements at 7, 3, and 1.3/0.85 mm and at optical wavelengths. The epochs of BIMA observations coincide with the epochs of corresponding VLBA images. See the legend for Fig. 1. 

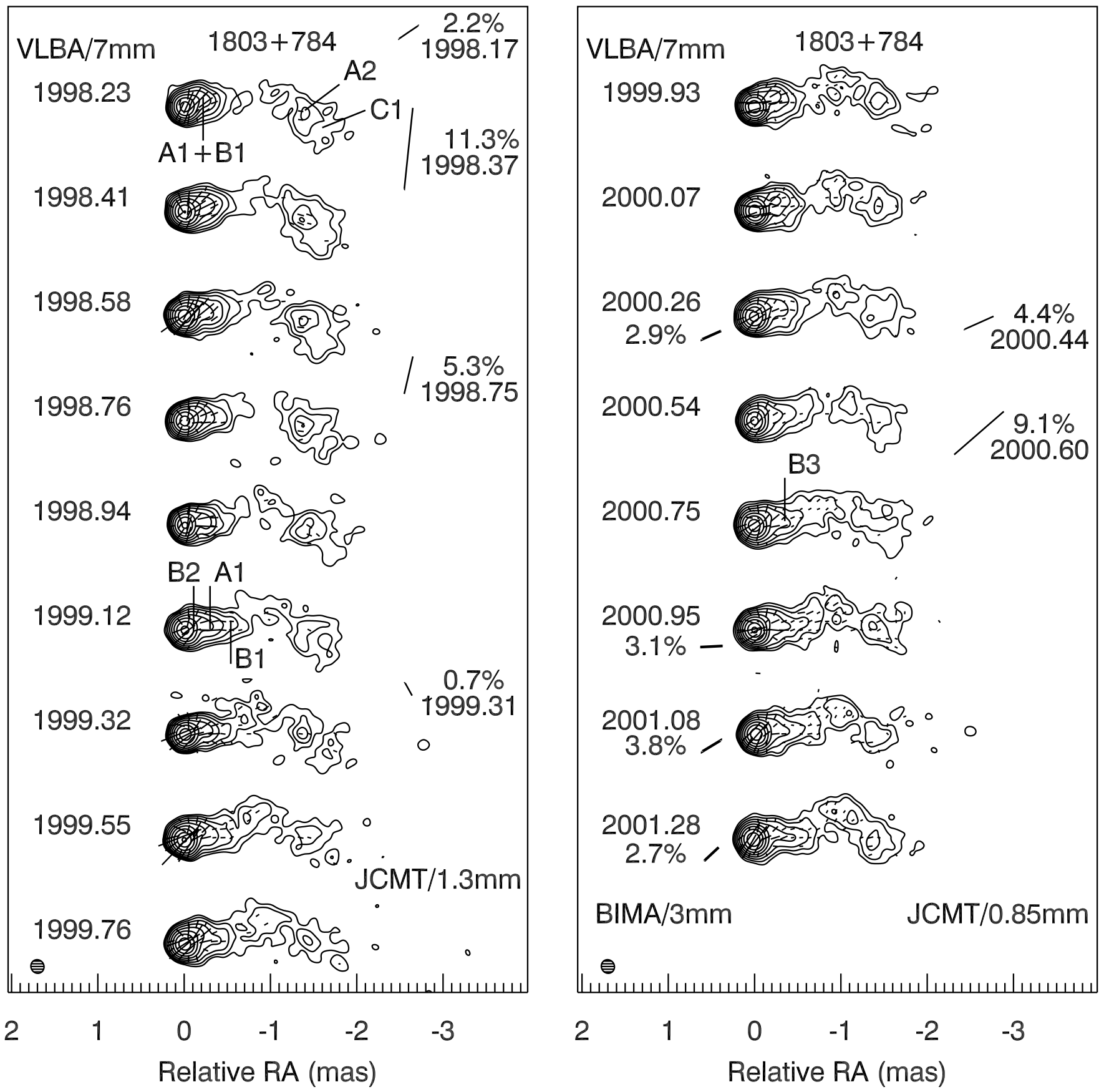

FIG. 11.-Total intensity images of $1803+784$ at $43 \mathrm{GHz}$ along with polarization measurements at 7,3 , and $1.3 / 0.85 \mathrm{~mm}$. The epochs of BIMA observations coincide with the epochs of corresponding VLBA images. See the legend for Fig. 1.

and optical observations were performed within 2 weeks of the corresponding VLBA epoch.

\subsection{Model Fitting of the VLBA Images}

We employed the task modelfit in DIFMAP to represent each total intensity image (Stokes parameter $I$ ) as a sequence of circular Gaussian components that are characterized by flux density, size, and position relative to the map center. Initially, pointlike components are used to obtain an image with 100:1 dynamic range. When a set of pointlike components was found, a final group of 100 iterations was executed with all parameters of all components allowed to vary to define their properties. In addition, because we had roughly bimonthly observations, we repeated the model fitting with the components from the previous epoch used as the initial model (except for the first epoch, 1998 March). This improves identification of components over epochs and provides an estimate of the accuracy of the parameters by comparison of the outcomes obtained with different initial models. For several sources (3C 66A, OJ 287, 3C 279, PKS 1510089, and 1803+784) at four epochs (1998 March, 1999 October, 2000 July, and 2001 January) we derived estimates of the $1 \sigma$ uncertainties of the best-fit models using the method described by Tzioumis et al. (1989) and realized in the package Difwrap (Lovell 2000). The uncertainties depend significantly on the brightness and size of the components: (1) for bright (flux of knot $\geq 100 \mathrm{rms}$ noise level) and compact (size $\leq 0.1$ mas) features, with uncertainties in flux density $\sim 1 \%$, in position $\sim 0.01$ mas, and in size $<1 \%$; (2) for the majority of components with size 

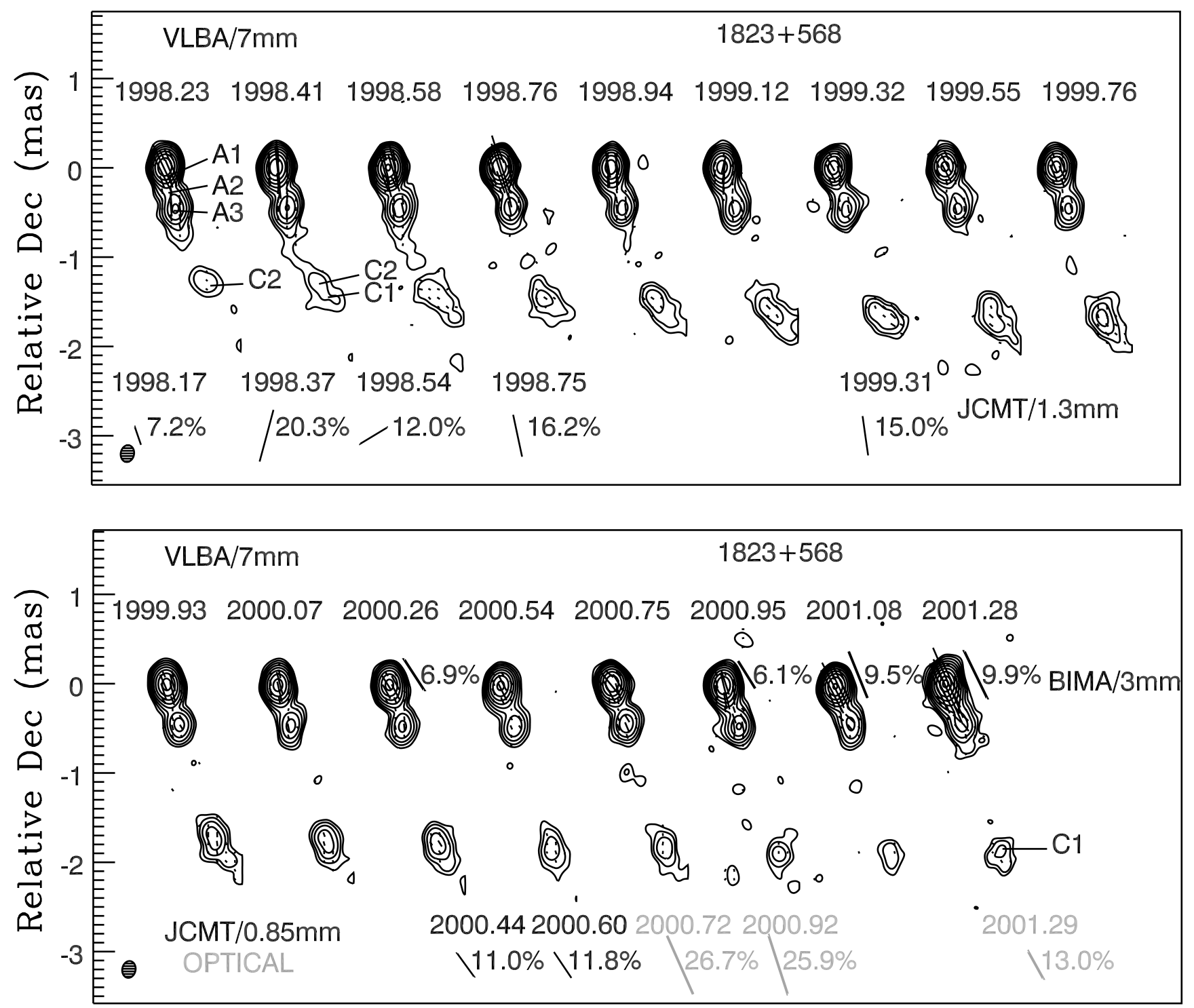

Fig. 12.-Total intensity images of $1823+568$ at $43 \mathrm{GHz}$ along with polarization measurements at 7,3 , and $1.3 / 0.85 \mathrm{~mm}$ and at optical wavelengths. The epochs of BIMA observations coincide with the epochs of corresponding VLBA images. See the legend for Fig. 1.

$0.1-0.3$ mas and flux $\geq 50 \mathrm{mJy}$, with uncertainties in the flux density $\sim 3 \%$, in position $\sim 1 / 5$ of the beam size, and in size $\sim 5 \%$; (3) for diffuse components, with uncertainties in flux density $\sim 10 \%$, in position comparable with half the size of the knot, and in size $\sim 10 \%$; (4) for components weaker than $50 \mathrm{mJy}$, with uncertainties in flux density $\sim 50 \%$ and positional uncertainties corresponding to the size of the average beam (see Table 1); however, for the majority of weak components these uncertainties were later modified as discussed in step 3 of $\S 4$.

We determined the parameters of polarized components by applying the task modelfit to the $(u, v)$ data in Stokes parameters $Q$ and $U$ separately. For this purpose only pointlike components were used in the models. Because the $Q$ and $U$ components could be either positive or negative, the procedure needed to be carried out carefully by checking with the polarized intensity image to avoid the inclusion of false features generated by the imaging procedure. The derived positional parameters of the $Q$ and $U$ components were compared with the parameters of $I$ components. We considered a $Q$ and/or $U$ component to be associated with an I component if they were cospatial to within the uncertainties. If both polarized components were present, the position of the po- larized component was defined by the average location, weighted by the absolute values of the $Q$ and $U$ fluxes. There are few cases in which a significant polarized component does not have an $I$ counterpart. Uncertainties in the values of the parameters of polarized components are difficult to define. Estimates of the accuracy of polarization parameters in jet features at 15 and $22 \mathrm{GHz}$ are discussed by Homan et al. (2002a). For components that are bright in the total intensity and highly polarized (percent polarization $\geq 5 \%$ ), we find that the uncertainties in fractional polarization are $\sim 1 \%$ and the uncertainties in polarization angle are $\sim 5^{\circ}$.

A table of parameters of jet features for each source over all epochs can be found online. ${ }^{12}$ The columns of these tables are as follows: (1) epoch, (2) flux density in janskys, (3) relative right ascension in milliarcseconds, (4) relative declination in milliarcseconds, (5) distance from the core in milliarcseconds, (6) position angle relative to the core in degrees, (7) angular size in milliarcseconds, (8) polarized flux density of a polarized component associated with a total intensity component, in janskys, (9) distance of the polarized component from the core in milliarcseconds,

\footnotetext{
${ }^{12}$ See http://www.bu.edu/blazars/multi.html.
} 

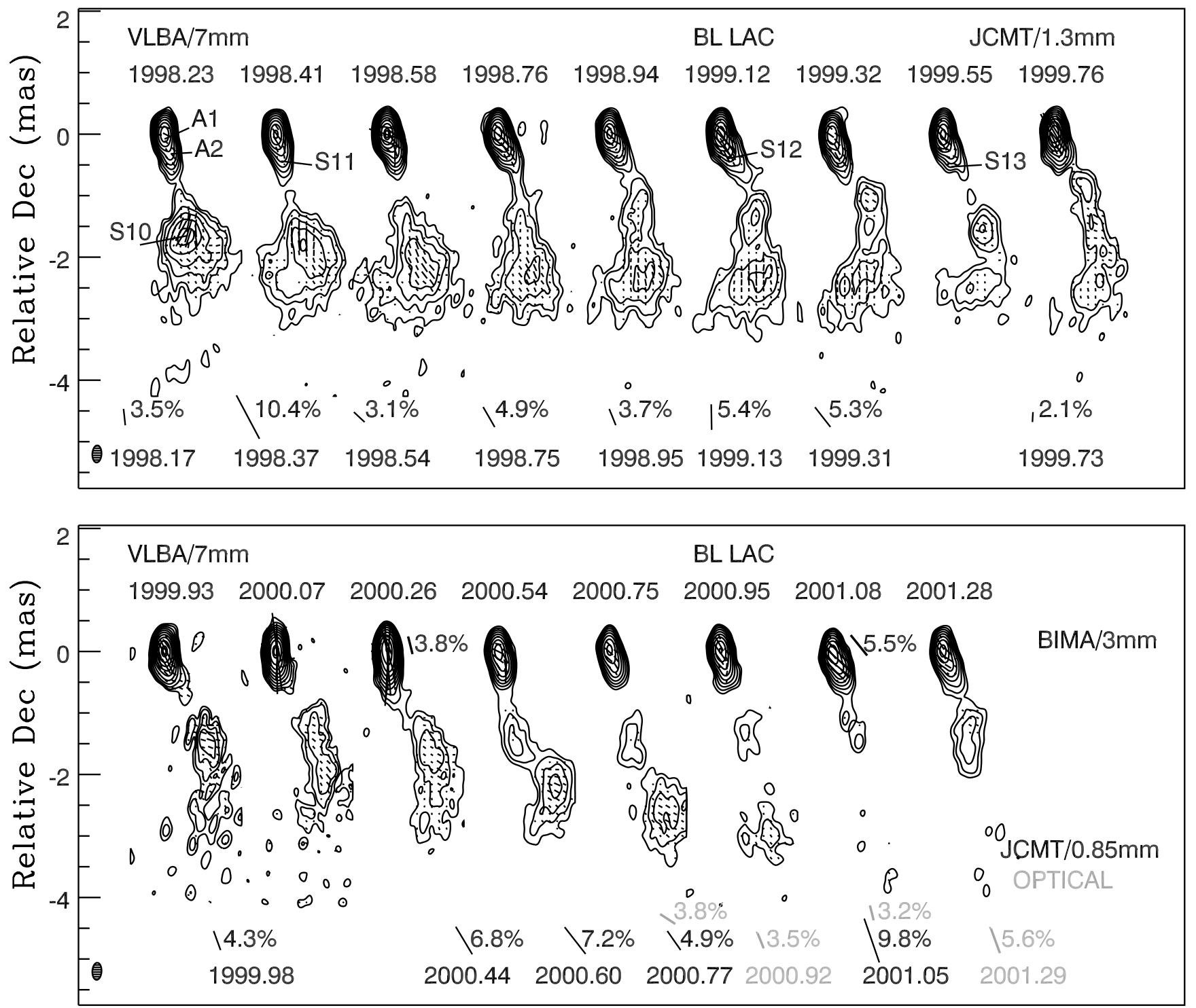

FIG. 13.- Total intensity images of BL Lac at $43 \mathrm{GHz}$ along with polarization measurements at 7,3 , and $1.3 / 0.85 \mathrm{~mm}$ and at optical wavelengths. The epochs of BIMA observations coincide with the epochs of corresponding VLBA images. See the legend for Fig. 1.

(10) position angle of the polarized component relative to the core in degrees, and (11) EVPA in degrees.

\section{TECHNIQUE TO MEASURE MOTION IN THE JETS}

The data allow us to follow the evolution of major features of the jets that are observed in the total and polarized intensity VLBA images of the 15 AGNs at 17 epochs over 3 years. For each image we identify a component, A0, as the VLBI core. For all sources A0 is located at one end of the jet, although it is not always the brightest feature. The position of the core in right ascension $x$ and declination $y$ is defined as $x=0, y=0$. In our analysis we assume that the core is stationary over all epochs. Locations of other features are determined relative to the core. Components are categorized as follows: knot A includes features (other than A0) that either are stationary (the proper motion is less than or equal to its uncertainty), undergo reverse motion (toward the core), or move at subluminal apparent speeds. Knots B, $\mathrm{C}$, and $\mathrm{D}$ are superluminal features, where $\mathrm{B}$ components are the fastest knots in the jet, D components are the farthest knots from the core, ejected before our monitoring period started, and the remainder are labeled as $\mathrm{C}$. The designation of components is different for 3C 120, 3C 279, 3C 345, and BL Lac, for which the naming corresponds to that of Gómez et al. (2001), Wehrle et al. (2001), Ros et al. (2000), and Stirling et al. (2003), respectively. Each component is characterized by the following parameters: total $(S)$ and polarized $\left(S_{p}\right)$ flux density; positions $x$ (right ascension), $y$ (declination), and $R$, where $R=\left(x^{2}+y^{2}\right)^{1 / 2}$; position angle $\Theta=\tan ^{-1}(x / y)$; size (FWHM) of the component, $a$; and EVPA. To define the temporal evolution of the jet and determine the apparent velocities of the jet flow we perform the following steps.

1. Identification of components at different epochs.-The identification of a component is based on a comparison of the parameters during the epochs when it is visible on the images. We assume that the same component has similar total $(S)$ and polarized $\left(S_{p}\right)$ fluxes, position angles $(\Theta)$ and EVPA, and size $(a)$ at successive epochs. However, some components evolve dramatically even over a 2 month interval between epochs, splitting into one or more subcomponents or merging with other features of the jet. In this paper we analyze those jet features whose 


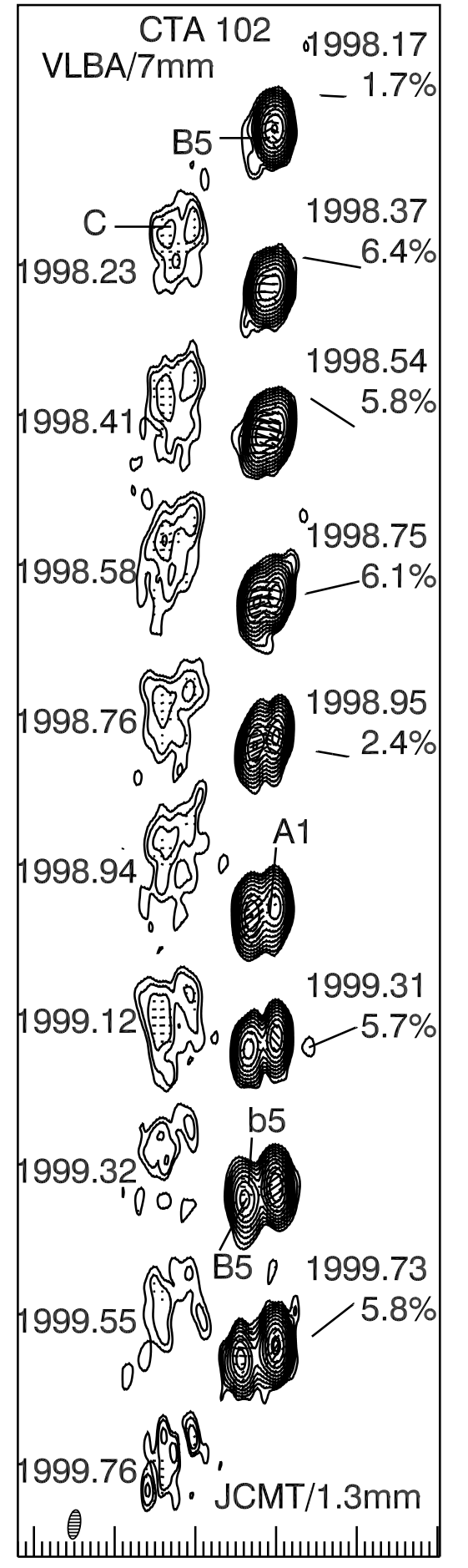

$\begin{array}{llllll}3 & 2 & 1 & 0 & -1 & -2\end{array}$

Relative RA (mas)

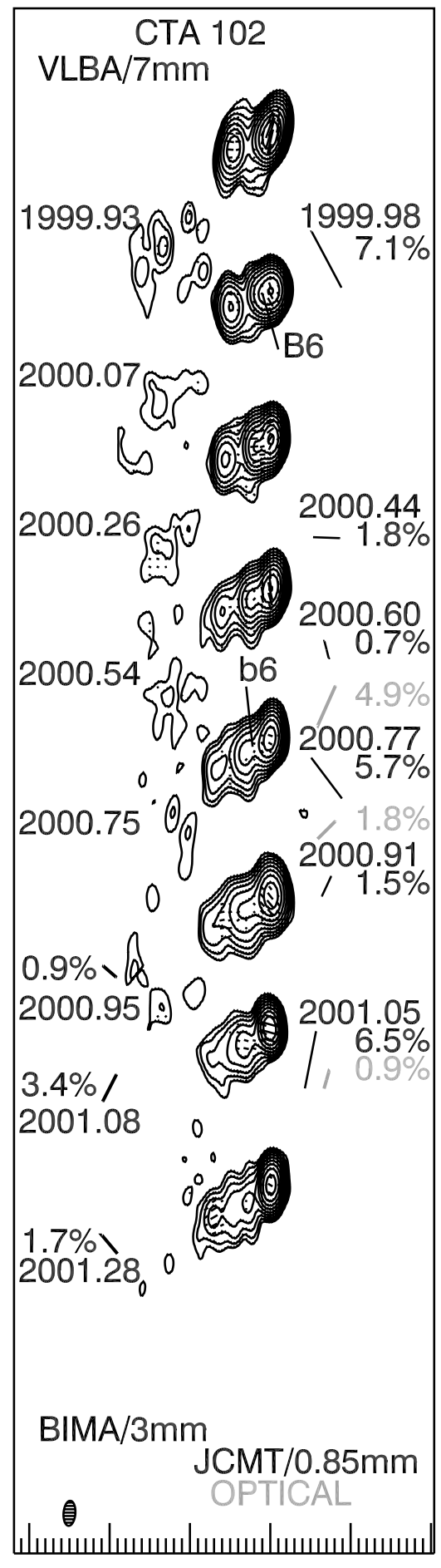

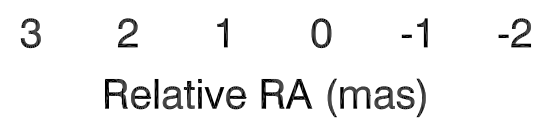

FIG. 14.-Total intensity images of CTA 102 at $43 \mathrm{GHz}$ along with polarization measurements at 7, 3, and 1.3/0.85 mm and at optical wavelengths. The epochs of BIMA observations coincide with the epochs of corresponding VLBA images. See the legend for Fig. 1. 

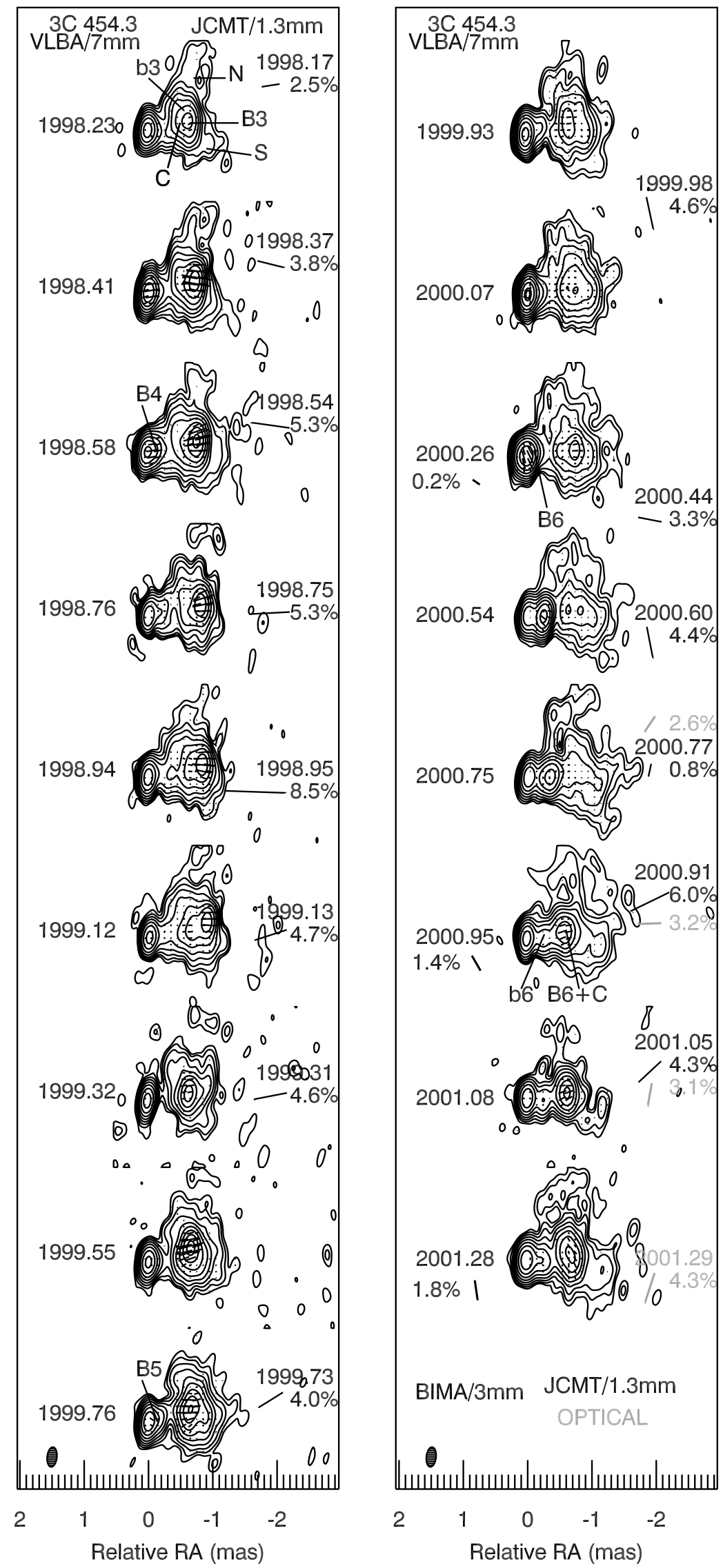

Fig. 15.-Total intensity images of $3 \mathrm{C} 454.3$ at $43 \mathrm{GHz}$ along with polarization measurements at 7,3 , and $1.3 / 0.85 \mathrm{~mm}$ and at optical wavelengths. The epochs of BIMA observations coincide with the epochs of corresponding VLBA images. See the legend for Fig. 1. 
TABLE 3

Best-Fit Polynomials for Component C1 of 3C 66A

\begin{tabular}{|c|c|c|c|c|c|c|c|c|c|c|c|c|c|c|c|c|}
\hline$l$ & $a_{0}$ & $a_{1}$ & $a_{2}$ & $a_{3}$ & $a_{4}$ & dof & $M$ & $M_{\chi^{2}}$ & $b_{0}$ & $b_{1}$ & $b_{2}$ & $b_{3}$ & $b_{4}$ & dof & $M$ & $M_{\chi^{2}}$ \\
\hline $0 \ldots \ldots \ldots \ldots$ & -0.1566 & $\ldots$ & $\ldots$ & $\ldots$ & $\ldots$ & 15 & 8.687 & 7.261 & -0.6114 & $\ldots$ & $\ldots$ & . . & $\ldots$ & 15 & 36.30 & 7.261 \\
\hline 1 ............... & -0.1575 & -0.0236 & $\ldots$ & $\ldots$ & $\ldots$ & 14 & 6.344 & 6.571 & -0.6164 & -0.1288 & $\ldots$ & . & $\ldots$ & 14 & 5.194 & 6.571 \\
\hline $2 \ldots \ldots \ldots \ldots$ & -0.1458 & -0.0233 & -0.0123 & $\ldots$ & $\ldots$ & 13 & 5.933 & 5.892 & -0.6410 & -0.1293 & -0.0258 & & $\ldots$ & 13 & 4.395 & 5.892 \\
\hline 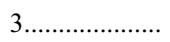 & -0.1448 & -0.0060 & -0.0129 & -0.0109 & $\ldots$ & 12 & 5.731 & 5.226 & -0.6421 & -0.1486 & 0.0264 & -0.0121 & $\ldots$ & 12 & 4.284 & 5.226 \\
\hline 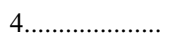 & -0.1327 & -0.0065 & -0.0577 & -0.0106 & 0.0201 & 11 & 5.261 & 4.575 & -0.6424 & -0.1486 & 0.0277 & -0.0121 & -0.0006 & 11 & 4.284 & 4.575 \\
\hline
\end{tabular}

TABLE 4

Best-Fit Polynomials for Component B1 of 3C 273

\begin{tabular}{|c|c|c|c|c|c|c|c|c|c|c|c|c|c|c|c|c|}
\hline$l$ & $a_{0}$ & $a_{1}$ & $a_{2}$ & $a_{3}$ & $a_{4}$ & dof & $M$ & $M_{\chi^{2}}$ & $b_{0}$ & $b_{1}$ & $b_{2}$ & $b_{3}$ & $b_{4}$ & dof & $M$ & $M_{\chi^{2}}$ \\
\hline $0 \ldots \ldots \ldots \ldots \ldots$ & -0.9252 & .. & $\ldots$ & $\ldots$ & $\ldots$ & 15 & 1009.0 & 7.261 & -0.7363 & $\ldots$ & $\ldots$ & $\ldots$ & $\ldots$ & 15 & 215.4 & 7.261 \\
\hline $1 \ldots \ldots$ & -1.384 & -0.6840 & $\ldots$ & $\ldots$ & $\ldots$ & 14 & 11.87 & 6.571 & -1.018 & -0.4385 & $\ldots$ & $\ldots$ & $\ldots$ & 14 & 7.262 & 6.571 \\
\hline $2 \ldots \ldots \ldots \ldots \ldots$ & -1.385 & -0.6361 & 0.0415 & .. & $\ldots$ & 13 & 10.13 & 5.892 & -1.029 & -0.3713 & 0.0683 & ... & $\ldots$ & 13 & 4.002 & 5.892 \\
\hline $3 \ldots \ldots \ldots \ldots$ & -1.367 & -0.5981 & -0.0114 & -0.0545 & $\ldots$ & 12 & 7.188 & 5.226 & -1.027 & -0.3657 & 0.0649 & -0.0051 & . & 12 & 3.988 & 5.226 \\
\hline 4 & -1.364 & -0.7077 & -0.1515 & 0.0150 & 0.0791 & 11 & 4.242 & 4.575 & -1.026 & -0.3740 & 0.0527 & -0.0001 & 0.0065 & 11 & 3.975 & 4.575 \\
\hline
\end{tabular}


TABLE 5

Jet Velocities

\begin{tabular}{|c|c|c|c|c|c|c|c|c|c|c|}
\hline Source & Knot & $N$ & $\begin{array}{c}\langle S\rangle \\
\text { (Jy) }\end{array}$ & $\begin{array}{c}\langle R\rangle \\
\text { (mas) }\end{array}$ & $\begin{array}{c}\langle\Theta\rangle \\
(\mathrm{deg})\end{array}$ & $l$ & $\begin{array}{c}\langle\Phi\rangle \\
(\operatorname{deg})\end{array}$ & $\begin{array}{c}\langle\mu\rangle \\
\left(\operatorname{mas} \mathrm{yr}^{-1}\right)\end{array}$ & $\begin{array}{c}\left\langle\beta_{\text {app }}\right\rangle \\
(c)\end{array}$ & $T_{0}$ \\
\hline \multirow{9}{*}{ 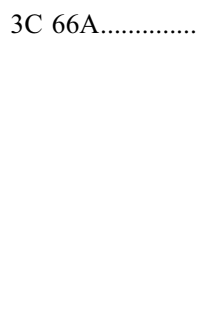 } & $\mathrm{C} 4$ & 6 & 0.20 & 0.12 & $-160.5 \pm 5.6$ & 1 & $-170.3 \pm 1.1$ & $0.06 \pm 0.04$ & $1.5 \pm 1.0$ & $1998.1 \pm 1.2$ \\
\hline & $\mathrm{C} 3$ & 12 & 0.10 & 0.23 & $-158.1 \pm 6.2$ & 1 & $-164.4 \pm 0.7$ & $0.12 \pm 0.02$ & $3.1 \pm 0.5$ & $1998.04 \pm 0.34$ \\
\hline & $\mathrm{C} 2$ & 16 & 0.06 & 0.37 & $-160.1 \pm 4.6$ & 1 & $-161.3 \pm 0.5$ & $0.19 \pm 0.01$ & $5.1 \pm 0.3$ & $1997.94 \pm 0.02$ \\
\hline & $\mathrm{C} 1$ & 16 & 0.03 & 0.63 & $-165.3 \pm 4.2$ & 1 & $-169.6 \pm 0.3$ & $0.13 \pm 0.01$ & $3.5 \pm 0.3$ & $1994.5 \pm 1.1$ \\
\hline & B7 & 5 & 0.03 & 0.48 & $-163.5 \pm 9.0$ & 1 & $-170.0 \pm 3.7$ & $0.98 \pm 0.13$ & $26.4 \pm 3.6$ & $1998.03 \pm 0.12$ \\
\hline & B6 & 11 & 0.02 & 1.55 & $-165.6 \pm 3.3$ & 1 & $-173.0 \pm 1.3$ & $0.81 \pm 0.06$ & $21.8 \pm 1.6$ & $1997.27 \pm 0.12$ \\
\hline & B4 & 7 & 0.03 & 2.04 & $-169.5 \pm 2.9$ & $1^{*}$ & $173.3 \pm 2.5$ & $1.00 \pm 0.12$ & $26.9 \pm 3.3$ & $1996.74 \pm 0.19$ \\
\hline & A1 & 16 & 0.02 & 1.19 & $-164.4 \pm 2.0$ & $1^{*}$ & $19.3 \pm 4.0$ & $0.14 \pm 0.10$ & $3.8 \pm 2.3$ & $\ldots$ \\
\hline & A2 & 17 & 0.05 & 2.50 & $-176.7 \pm 2.2$ & $1^{*}$ & $-42.3 \pm 6.2$ & $0.09 \pm 0.06$ & $2.5 \pm 1.5$ & $\ldots$ \\
\hline
\end{tabular}

Noте.-Table 5 is published in its entirety in the electronic edition of the Astronomical Journal. A portion is shown here for guidance regarding its form and content.

identification is supported by the similarity of a number of parameters at different epochs.

2. Fitting various polynomials.-The thorough sequences of images allow us to search for acceleration/deceleration of the jet flow and nonballistic projected trajectories. We fit the $(x, y)$ positions of a component over $N$ epochs by different polynomials of order $l$ :

$$
x\left(t_{i}\right)=a_{0}+a_{1}\left(t_{i}-t_{\text {mid }}\right)+a_{2}\left(t_{i}-t_{\text {mid }}\right)^{2}+\ldots a_{l}\left(t_{i}-t_{\text {mid }}\right)^{l},
$$

$$
y\left(t_{i}\right)=b_{0}+b_{1}\left(t_{i}-t_{\text {mid }}\right)+b_{2}\left(t_{i}-t_{\text {mid }}\right)^{2}+\ldots b_{l}\left(t_{i}-t_{\text {mid }}\right)^{l},
$$

where $t_{i}$ is the epoch of observation, $i=1, \ldots, N$, and $t_{\text {mid }}=$ $\left(t_{1}+t_{N}\right) / 2$. We use the program LSQPOL of the FORTRAN version of the package DATAN (Brandt 1999) to find the optimal polynomials of order $l$, where $l$ runs from 0 to 4 . The upper limit, $l=4$, is a consequence of the maximum number of epochs of observation of a knot, equal to 17 . The program provides the value $M_{l}$, which is the goodness of fit by a polynomial of order $l$. For each order, we perform a $\chi^{2}$ test to determine the polynomial that best fits the data. We choose a polynomial of the lowest order for which $M_{l}<M_{\chi^{2}}$, where $M_{\chi^{2}}$ is the value of the $\chi^{2}$ distribution corresponding to significance level $\zeta=0.05$ for dof $=N-l-1$ degrees of freedom (Bowker \& Lieberman 1972).

Examples of the selection of polynomials that fit the data are given in Tables 3 and 4 for component $\mathrm{C} 1$ identified in the jet of $3 \mathrm{C} 66 \mathrm{~A}$ at 16 epochs and component $\mathrm{B} 1$, also seen at 16 epochs, in $3 \mathrm{C} 273$. These tables show parameters of the best-fit polynomials of order $0-4$, the corresponding values of the goodness of fit to the data $M$, and the goodness of fit required by the $\chi^{2}$ test. Table 3 shows that $l=1$ satisfies the $\chi^{2}$ test for coordinates $x$ and $y$ in the case of $\mathrm{C} 1$ for $3 \mathrm{C} 66 \mathrm{~A}$. For $\mathrm{B} 1, l=4$ is required to match the motion in right ascension, while a second-order polynomial adequately describes the data for declination.

TABLE 6

\begin{tabular}{|c|c|c|c|c|c|c|}
\hline Source & Knot & $N$ & $\begin{array}{c}\langle\mu\rangle \\
\left(\operatorname{mas} \mathrm{yr}^{-1}\right)\end{array}$ & $\begin{array}{c}\langle\Phi\rangle \\
(\mathrm{deg})\end{array}$ & $\begin{array}{c}\dot{\mu}_{\|} \\
\left(\operatorname{mas} \mathrm{yr}^{-2}\right)\end{array}$ & $\begin{array}{c}\dot{\mu}_{\perp} \\
\left(\text { mas yr }^{-2} \text { ) }\right.\end{array}$ \\
\hline 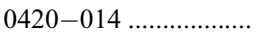 & B1 & 14 & $0.22 \pm 0.06$ & $171.8 \pm 2.2$ & $0.07 \pm 0.02$ & $0.02 \pm 0.01$ \\
\hline $3 \mathrm{C} 111 .$. & $\mathrm{c} 1$ & 16 & $1.07 \pm 0.02$ & $56.9 \pm 0.9$ & $-0.22 \pm 0.02$ & $-0.26 \pm 0.02$ \\
\hline \multirow[t]{2}{*}{ 3C $120 \ldots \ldots \ldots \ldots \ldots \ldots \ldots$} & o1 & 16 & $2.04 \pm 0.06$ & $-118.6 \pm 2.8$ & $0.29 \pm 0.02$ & $0.16 \pm 0.02$ \\
\hline & $\mathrm{o} 2$ & 14 & $1.52 \pm 0.03$ & $-119.1 \pm 1.4$ & $-0.66 \pm 0.03$ & $-0.12 \pm 0.04$ \\
\hline \multirow[t]{3}{*}{$0528+134 \ldots \ldots \ldots \ldots \ldots \ldots$} & B8 & 11 & $0.11 \pm 0.02$ & $44.3 \pm 1.0$ & $0.10 \pm 0.01$ & $-0.07 \pm 0.02$ \\
\hline & B6 & 13 & $0.26 \pm 0.04$ & $52.9 \pm 2.2$ & $0.06 \pm 0.03$ & $-0.17 \pm 0.04$ \\
\hline & B4 & 9 & $0.21 \pm 0.08$ & $36.6 \pm 5.6$ & $0.11 \pm 0.02$ & $-0.09 \pm 0.03$ \\
\hline \multirow[t]{2}{*}{ 3C $273 \ldots \ldots \ldots \ldots \ldots \ldots \ldots \ldots$} & B1 & 16 & $0.79 \pm 0.06$ & $-120.3 \pm 3.0$ & $-0.14 \pm 0.03$ & $0.10 \pm 0.02$ \\
\hline & B2 & 10 & $1.18 \pm 0.06$ & $-123.5 \pm 3.3$ & $0.04 \pm 0.03$ & $0.26 \pm 0.04$ \\
\hline \multirow[t]{2}{*}{ 3С $279 \ldots \ldots \ldots \ldots \ldots \ldots \ldots$} & $\mathrm{C} 8^{*}$ & 27 & $0.33 \pm 0.01$ & $-126.6 \pm 0.7$ & $0.07 \pm 0.01$ & $0.02 \pm 0.01$ \\
\hline & $\mathrm{C} 4$ & 46 & $0.31 \pm 0.01$ & $-127.9 \pm 0.8$ & $0.02 \pm 0.01$ & $-0.05 \pm 0.01$ \\
\hline \multirow[t]{4}{*}{ 3C $345 \ldots \ldots \ldots \ldots \ldots \ldots \ldots \ldots$} & C11 & 10 & $0.69 \pm 0.03$ & $-94.5 \pm 0.4$ & $0.22 \pm 0.03$ & $0.19 \pm 0.04$ \\
\hline & C9 & 17 & $0.45 \pm 0.04$ & $-79.1 \pm 1.0$ & $0.11 \pm 0.02$ & $-0.08 \pm 0.03$ \\
\hline & $\mathrm{C} 8$ & 10 & $0.32 \pm 0.10$ & $-65.6 \pm 4.7$ & $-0.20 \pm 0.03$ & $-0.23 \pm 0.03$ \\
\hline & $\mathrm{C} 7$ & 16 & $0.29 \pm 0.02$ & $-81.4 \pm 0.3$ & $-0.07 \pm 0.01$ & $-0.01 \pm 0.01$ \\
\hline \multirow[t]{2}{*}{$1803+784 \ldots \ldots \ldots \ldots \ldots \ldots . .}$. & B2 & 13 & $0.42 \pm 0.05$ & $-75.4 \pm 1.6$ & $0.30 \pm 0.05$ & $-0.10 \pm 0.04$ \\
\hline & B1 & 14 & $0.42 \pm 0.05$ & $-60.0 \pm 2.4$ & $0.52 \pm 0.04$ & $-0.29 \pm 0.03$ \\
\hline \multirow[t]{3}{*}{ BL Lac .......................... } & S13 & 10 & $1.95 \pm 0.11$ & $-170.2 \pm 2.9$ & $-0.21 \pm 0.07$ & $-0.76 \pm 0.06$ \\
\hline & S11 & 10 & $1.36 \pm 0.13$ & $-167.1 \pm 4.2$ & $0.07 \pm 0.06$ & $-0.62 \pm 0.05$ \\
\hline & $\mathrm{S} 10$ & 10 & $0.91 \pm 0.03$ & $-175.8 \pm 2.4$ & $-0.32 \pm 0.03$ & $-0.71 \pm 0.04$ \\
\hline \multirow[t]{2}{*}{ 3C $454.3 \ldots \ldots \ldots \ldots \ldots \ldots \ldots$} & B6 & 7 & $0.53 \pm 0.05$ & $-79.5 \pm 1.2$ & $0.37 \pm 0.06$ & $0.24 \pm 0.07$ \\
\hline & B4 & 15 & $0.41 \pm 0.02$ & $-98.3 \pm 0.4$ & $-0.04 \pm 0.01$ & $-0.09 \pm 0.02$ \\
\hline
\end{tabular}

Acceleration in the Jets 

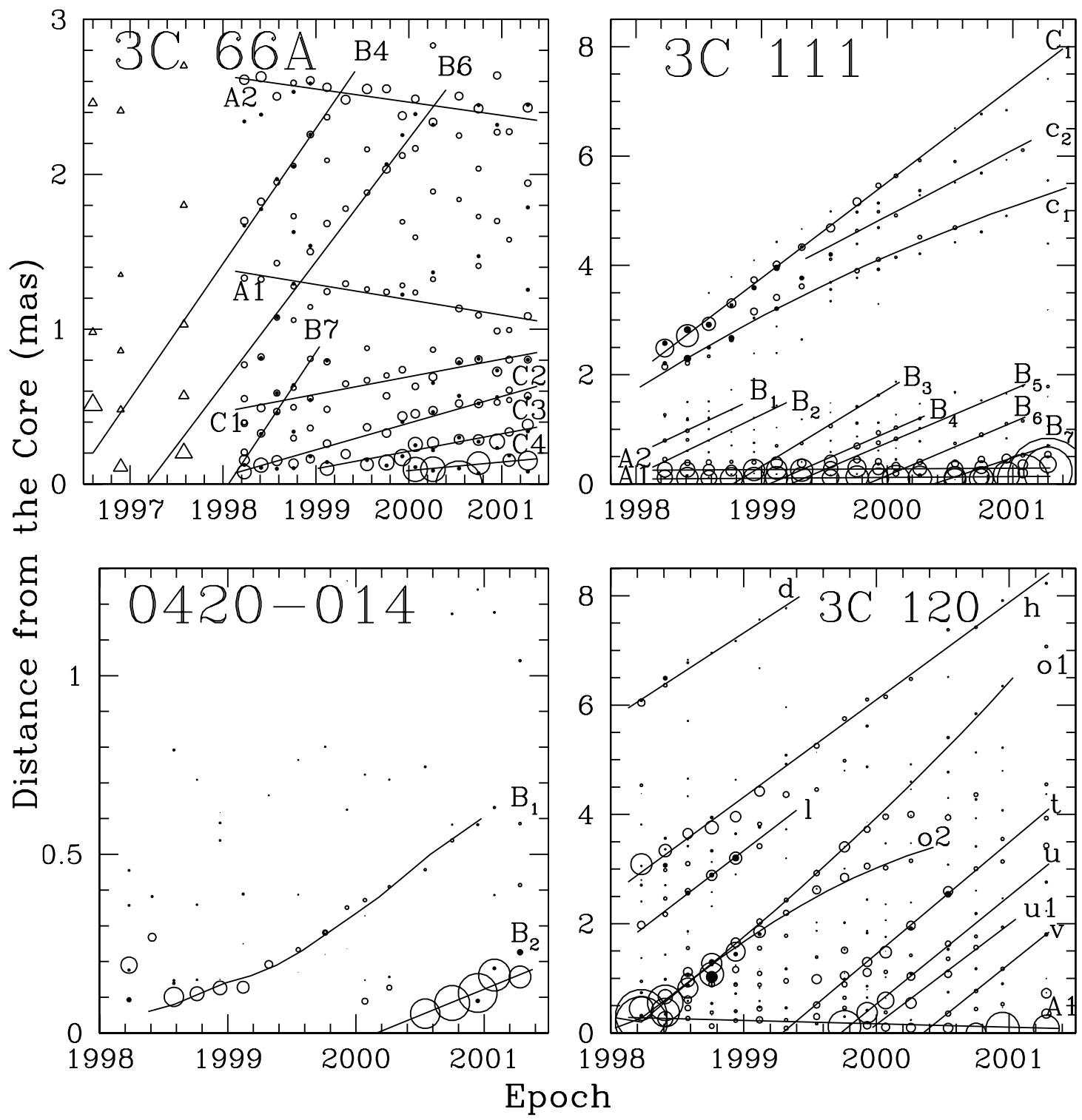

FIG. 16. - Separation of components from the VLBA core vs. epoch of observation. Total intensity components are indicated by open circles and polarized intensity components by filled circles. In a few cases (3C 66A, 0528+134, 3C 279, and 3C 345) data prior to 1998 are from Jorstad et al. (2001b; triangles), Wehrle et al. (2001; squares), and Ros et al. (2000; asterisks) (see the Appendix for details). For 3C 279 and 3C 454.3 different symbols are used to separate closely located components. The size of a symbol is related to the flux density. The solid lines and curves represent the best polynomial approximation to the data according to Table 5. The designation of knots follows Table 5 .

In a few cases the $\chi^{2}$ test is not satisfied even by polynomials of order 4. An increase in the order of the polynomial does not improve the situation, since, given the limited number of observations, the number of degrees of freedom becomes too small. Such cases are approximated by a straight line with special consideration for uncertainties in the $(x, y)$ values (see below). These cases are marked in Table 5 by asterisks in the polynomial order (l) column.

3. Uncertainties in polynomial parameters.-After the bestfit polynomial is found, we run the subroutine LSQASN of the package DATAN to estimate errors in the derived parameters. This method is valid for a normal distribution of the unknowns and when the true value lies with probability $W$ within the confidence region around the estimated value ( $W=0.95$ is used). However, the errors depend significantly on the uncertainties in the individual observations that are obtained from the model fitting (see $\S 3$ ). This allows us to lessen the weight of data at epochs with bad weather or a failure of one or more antennas, or when a given component was very weak or diffuse. For component motion that cannot be represented well by polynomials $l \leq 4$ (see above), we approximate the motion by a first-order polynomial and determine uncertainties in the $(x, y)$ position using the program LSQPOL and the method suggested by Homan et al. (2001). We set the uncertainty for each data point equal to the average beam size and calculate the optimal polynomial of first order corresponding to a preliminary $\chi^{2}$ value. Taking this preliminary $\chi^{2}$ value, we uniformly rescale the uncertainties in the data points such that $\chi^{2}$ would correspond to the $\chi^{2}$ value for $\zeta=0.05$ and dof $=N-2$.

4. Calculation of proper motion, acceleration, and ejection time.-We define the average proper motion as a vector $(\langle\mu\rangle$, $\langle\Phi\rangle)$, where $\langle\mu\rangle$ represents the mean angular speed of motion and $\langle\Phi\rangle$ gives the average direction of motion. The average values are derived as follows: $\langle\mu\rangle=\left(\left\langle\mu_{x}\right\rangle^{2}+\left\langle\mu_{y}\right\rangle^{2}\right)^{1 / 2}$ and $\langle\Phi\rangle=$ $\tan ^{-1}\left(\left\langle\mu_{x}\right\rangle /\left\langle\mu_{y}\right\rangle\right)$, where $\left\langle\mu_{x}\right\rangle=\int_{t_{1}}^{t_{N}} \dot{x} d t /\left(t_{N}-t_{1}\right)$ and $\left\langle\mu_{y}\right\rangle=$ $\int_{t_{1}}^{t_{N}} \dot{y} d t /\left(t_{N}-t_{1}\right)$. In the case of first- or second-order polynomi- 

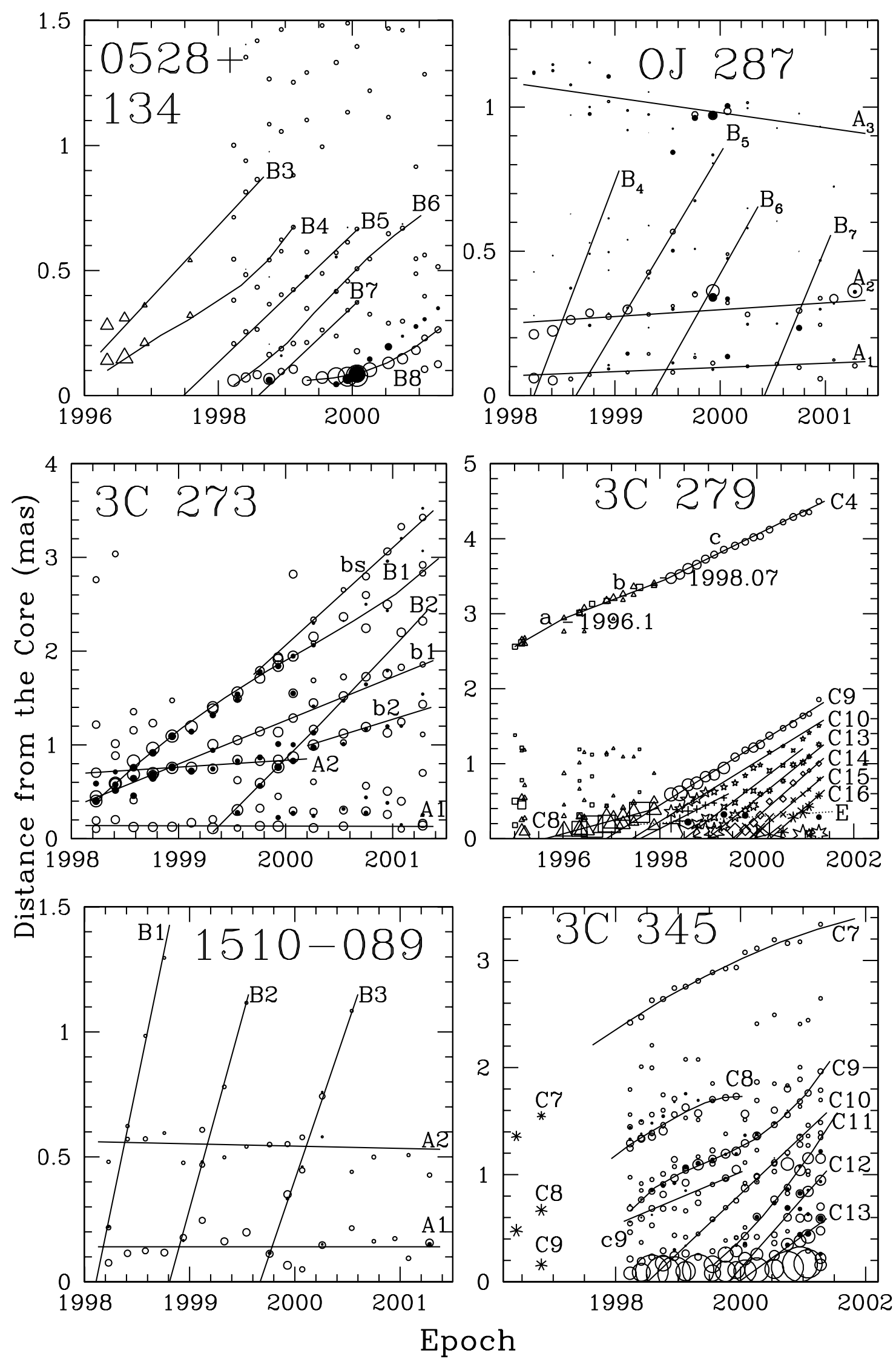

Fig. 16.-Continued 

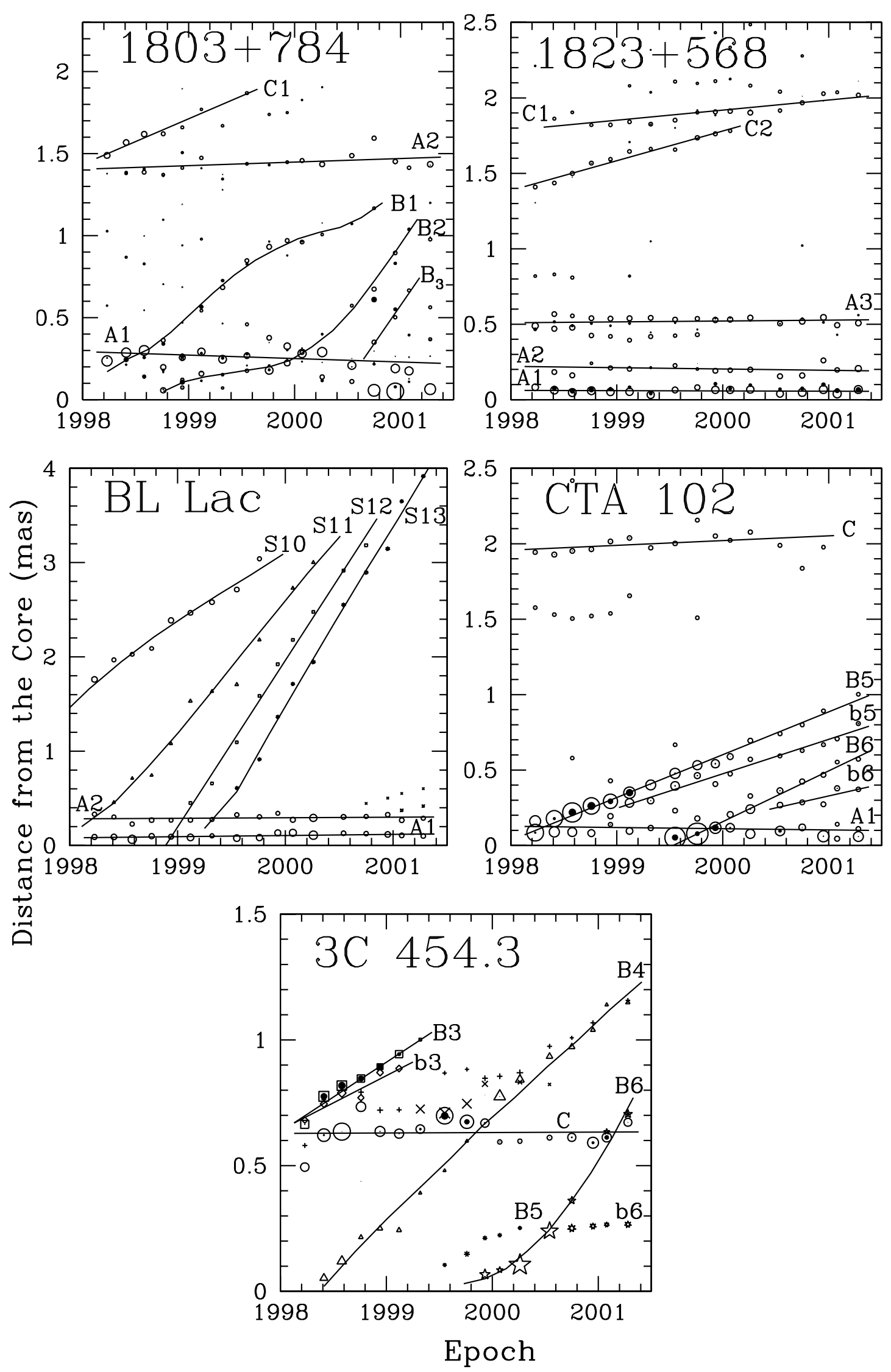

FIg. 16.-Continued 

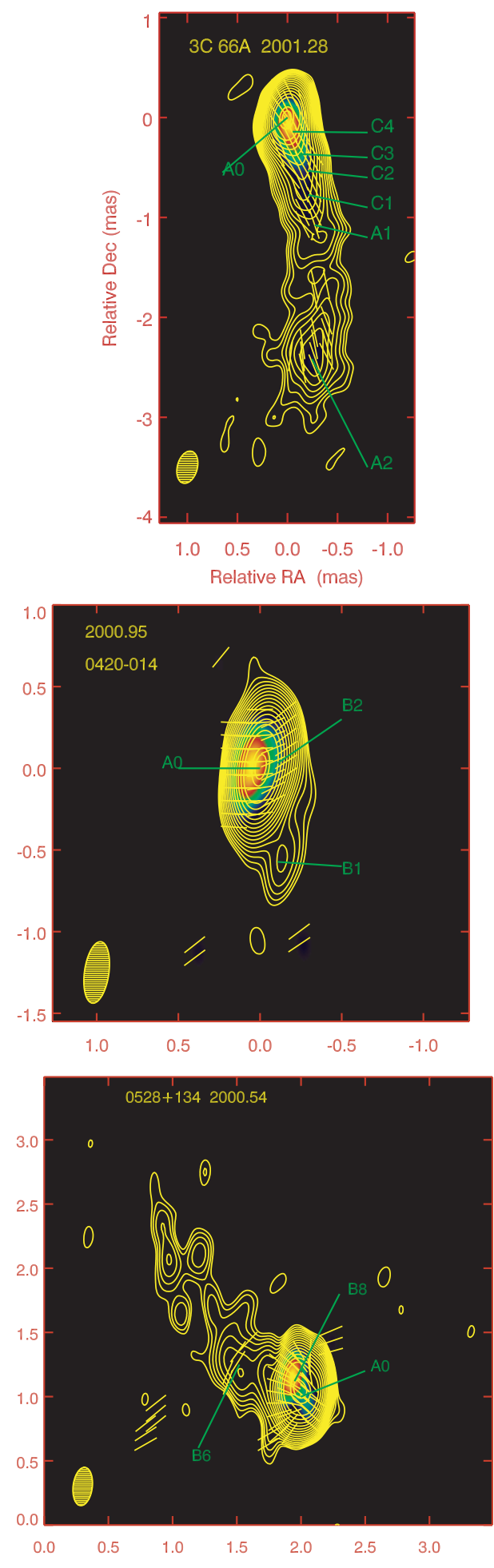
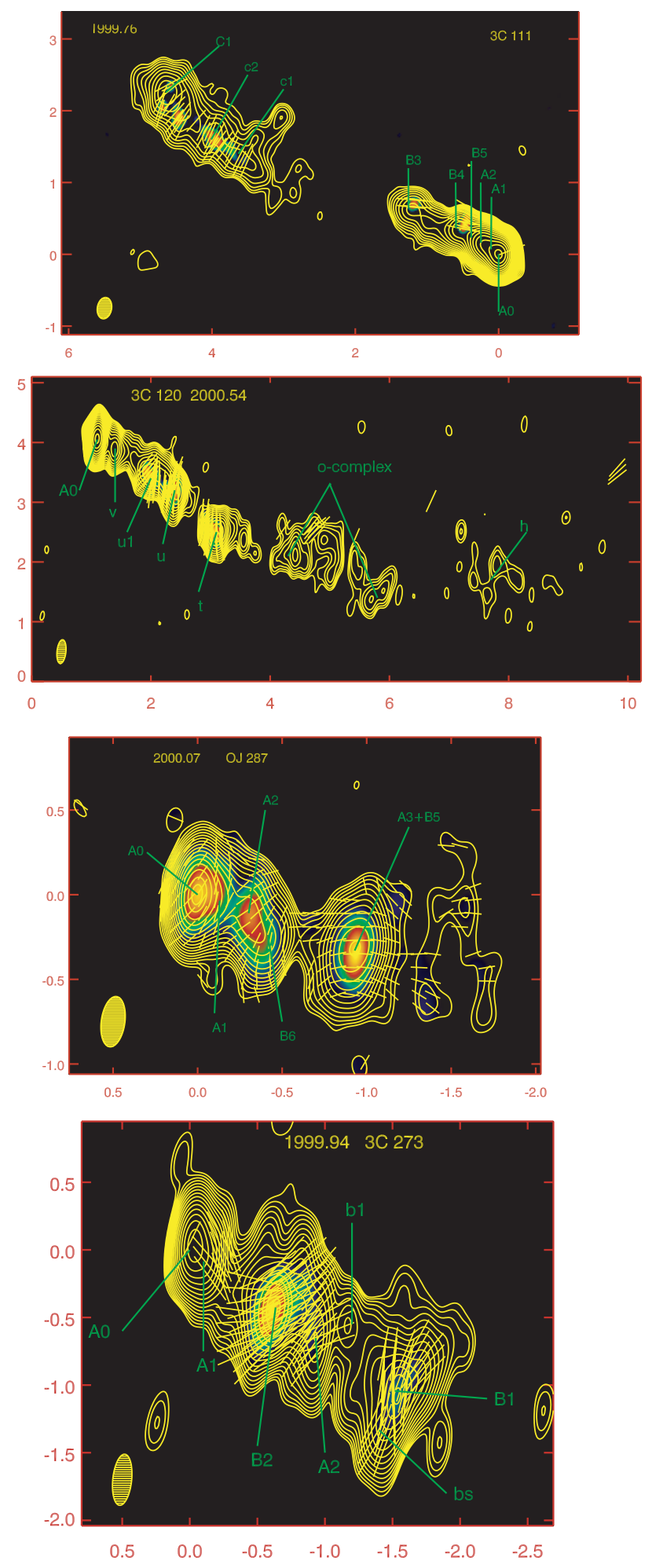

Fig. 17.- Total (contours) and polarized (color scale) intensity images at $43 \mathrm{GHz}$. The yellow line segments show the direction of the electric vectors. Parameters of the maps are given in Table 7. The designation of components follows Table 5 and Fig. 16. 

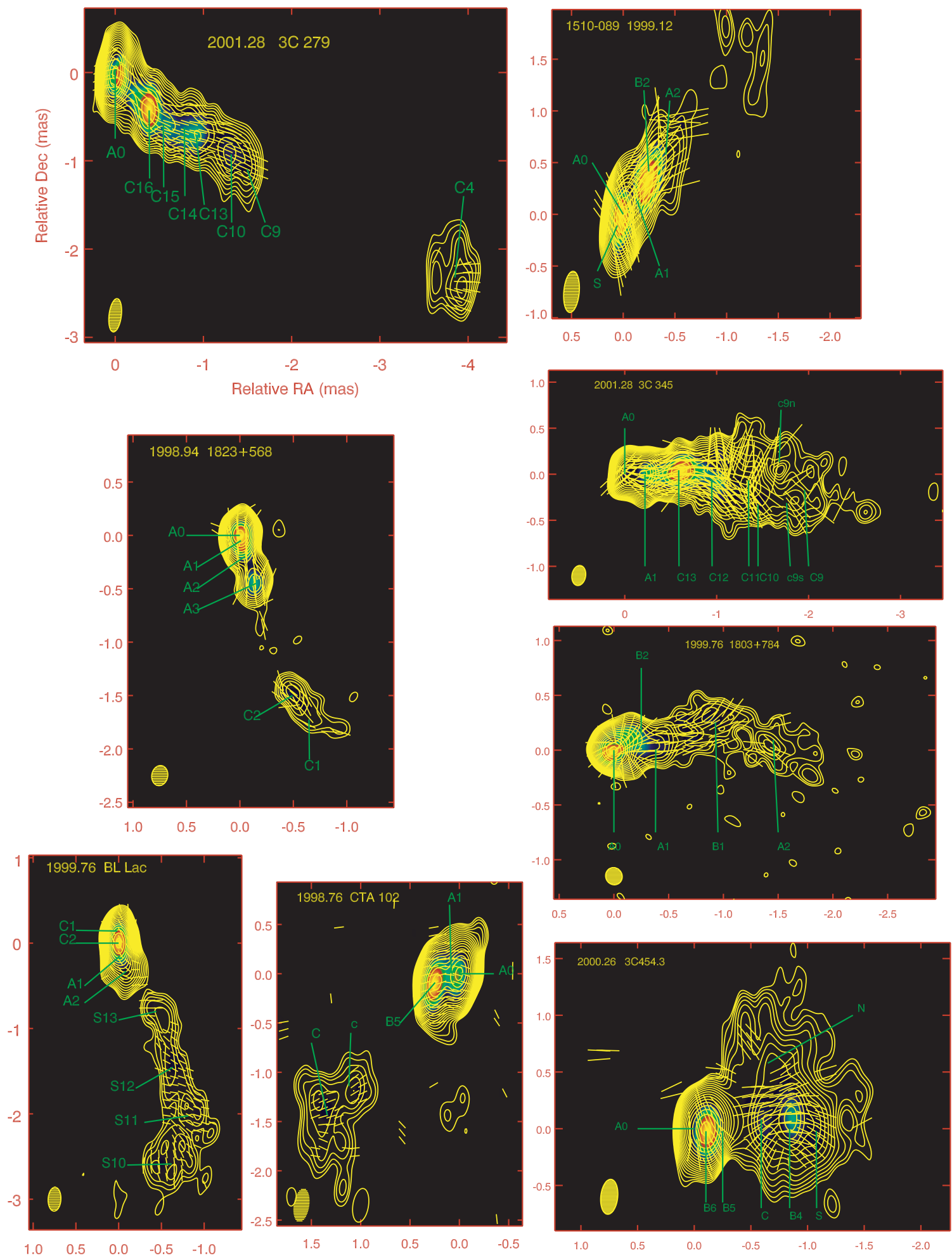

FIG. 17.-Continued 
TABLE 7

Parameters of Total and Polarized Intensity Maps Shown in Fig. 17

\begin{tabular}{|c|c|c|c|c|c|c|c|}
\hline Source & $\begin{array}{l}\text { Beam } \\
\text { (mas) }\end{array}$ & $\begin{array}{l}\text { P.A. } \\
\text { (deg) }\end{array}$ & $\begin{array}{c}I_{\text {peak }} \\
\left(\mathrm{mJy} \mathrm{beam}^{-1}\right)\end{array}$ & $\begin{array}{c}I_{\text {peak }}^{p} \\
\left(\mathrm{mJy} \mathrm{beam}^{-1}\right)\end{array}$ & $\begin{array}{c}I_{\min } \\
\left(\mathrm{mJy} \mathrm{beam}^{-1}\right)\end{array}$ & $\begin{array}{c}\operatorname{rms}(I) \\
\left(\mathrm{mJy} \mathrm{beam}^{-1}\right)\end{array}$ & $\begin{array}{c}\operatorname{rms}\left(I^{p}\right) \\
\left(\mathrm{mJy} \mathrm{beam}^{-1}\right)\end{array}$ \\
\hline 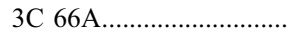 & $0.32 \times 0.19$ & -13 & 500 & 30 & 1.5 & 0.7 & 0.5 \\
\hline 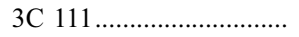 & $0.30 \times 0.18$ & -10 & 803 & 6 & 2.0 & 0.9 & 0.9 \\
\hline 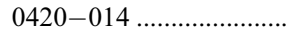 & $0.36 \times 0.14$ & -8 & 3504 & 112 & 10.5 & 4.0 & 2.7 \\
\hline 3C $120 \ldots \ldots \ldots \ldots \ldots \ldots \ldots \ldots . .$. & $0.38 \times 0.15$ & -6 & 650 & 11 & 2.9 & 0.7 & 1.4 \\
\hline 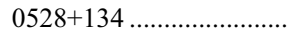 & $0.30 \times 0.15$ & -7 & 1426 & 53 & 3.8 & 1.8 & 1.2 \\
\hline 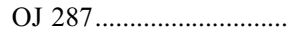 & $0.30 \times 0.14$ & -8 & 1115 & 32 & 4.4 & 2.5 & 2.6 \\
\hline 3С $273 \ldots \ldots \ldots \ldots \ldots \ldots \ldots \ldots \ldots$ & $0.42 \times 0.17$ & -6 & 7178 & 655 & 35.9 & 7.6 & 14.1 \\
\hline 3С $279 \ldots \ldots \ldots \ldots \ldots \ldots \ldots \ldots$ & $0.38 \times 0.14$ & -7 & 9695 & 432 & 24.2 & 3.5 & 3.1 \\
\hline 1510-089 …..................... & $0.40 \times 0.15$ & -5 & 1517 & 36 & 7.6 & 1.5 & 3.4 \\
\hline 3C $345 \ldots \ldots \ldots \ldots \ldots \ldots \ldots \ldots \ldots \ldots \ldots$ & $0.22 \times 0.15$ & -10 & 1470 & 144 & 7.4 & 1.2 & 1.4 \\
\hline $1803+784 \ldots \ldots \ldots \ldots \ldots \ldots \ldots$ & $0.16 \times 0.15$ & 30 & 1211 & 61 & 4.2 & 1.3 & 2.2 \\
\hline $1823+568 \ldots \ldots \ldots \ldots \ldots \ldots \ldots \ldots$ & $0.19 \times 0.15$ & -11 & 781 & 55 & 3.9 & 0.6 & 1.4 \\
\hline BL Lac ............................. & $0.28 \times 0.15$ & -4 & 2709 & 128 & 6.8 & 2.2 & 2.7 \\
\hline 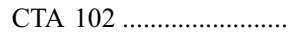 & $0.24 \times 0.16$ & -9 & 2456 & 109 & 6.1 & 3.1 & 4.6 \\
\hline 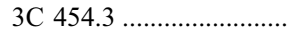 & $0.30 \times 0.15$ & -6 & 3729 & 206 & 9.3 & 1.8 & 2.9 \\
\hline
\end{tabular}

als, $\left\langle\mu_{x}\right\rangle=a_{1}$ and $\left\langle\mu_{y}\right\rangle=b_{1}$. For a polynomial of order higher than unity we compute an average vector of acceleration, $(\dot{\mu} \|$, $\dot{\mu}_{\perp}$ ), where $\dot{\mu}_{\|}$is along the direction of the average velocity, $\langle\Phi\rangle$. In the case of a second-order polynomial, $\dot{\mu}_{x}=2 a_{2}$ and $\dot{\mu}_{y}=$ $2 b_{2}$. For a higher order polynomial, $\dot{\mu}_{x}=\int_{t_{1}}^{t_{N}} \ddot{x} d t /\left(t_{N}-t_{1}\right)$ and $\dot{\mu}_{y}=\int_{t_{1}}^{t_{N}} \ddot{y} d t /\left(t_{N}-t_{1}\right)$.

\section{JET VELOCITIES}

We find superluminal apparent speeds for 19 of 22 components in the radio galaxies, 19 of 31 knots in the BL Lac objects, and 46 of 53 knots in the quasars. Table 5 lists the average apparent speed, $\left\langle\beta_{\text {app }}\right\rangle$, calculated in units of the speed of light $c$ and using the average proper motion $\langle\mu\rangle$. An inhomogeneous Friedmann-Lemaitre-Robertson-Walker cosmology, with $\Omega_{m}=$ $0.3, \Omega_{\Lambda}=0.7$, and Hubble constant $H_{0}=70 \mathrm{~km} \mathrm{~s}^{-1} \mathrm{Mpc}^{-1}$ (Kantowski et al. 2000), is adopted for the calculations. We derive the uncertainties in the average proper motion, acceleration, and apparent speed from the uncertainties in the polynomial coefficients. Table 5 also contains the number of epochs, $N$, at which the component has been observed; the average total flux, $\langle S\rangle$, in janskys; the average distance from the core, $\langle R\rangle$, in milliarcseconds; the average position angle of the component, $\langle\Theta\rangle$, in degrees, with uncertainty corresponding to the scatter across the epochs; the average direction of the velocity vector, $\langle\Phi\rangle$, in degrees; and the ejection time (epoch of zero separation), $T_{0}$. The ejection time is the extrapolated time of coincidence of the position of a moving knot with the core in the VLBA images, and $T_{0}$ is the average of $t_{x, 0}$ and $t_{y, 0}$ weighted by their uncertainties, where $t_{x, 0}$ and $t_{y, 0}$ are roots of the best-fit polynomials. In the case of a polynomial of order 3 or higher, the roots are computed by successive iterations, with an accuracy of $10^{-5}$.

Table 6 gives the parameters of acceleration for components in Table 5 having best-fit polynomials of order $\geq 2$ (some columns repeat information from Table 5 for convenience). Figure 16 shows the positions of the jet components relative to the core for both the total (open circles) and polarized ( filled circles) intensity images of each source. The solid lines/curves indicate the best polynomial fit to the data. Components presented in Figure 16 are marked at the first epoch in Figures 1-15 if they are detected then or at the later epoch at which they appear in the jet. Figure 17 displays an image from one particular epoch for each source when the most prominent jet features are seen. Table 7 lists the param- eters of the maps shown in Figure $17\left[\operatorname{rms}(I)\right.$ and $\operatorname{rms}\left(I^{p}\right)$ are the rms values of the residual total and polarized intensity on the images, respectively]. The trajectories of all components with nonballistic motion are plotted in Figure 18. The Appendix contains a description of our results for each individual object in the sample.

\section{APPARENT SPEED AS A PROBE OF PARSEC-SCALE JETS}

Our intensive, prolonged, high-frequency VLBI monitoring reveals complex, changing structure for the parsec-scale jets, including significant variations in the apparent speed of knots in the jets, $\beta_{\text {app }}$, within individual objects. The apparent speed is determined by the intrinsic velocity, $\beta$, and the angle between the trajectory and the line of sight, $\Theta_{0}$ :

$$
\beta_{\text {app }}=\beta \sin \Theta_{0}\left(1-\beta \cos \Theta_{0}\right)^{-1}
$$

The intrinsic velocity defines the Lorentz factor of the knot, $\Gamma=$ $1 /\left(1-\beta^{2}\right)^{1 / 2}$, where $\beta$ is in units of the speed of light. Large scatter in the apparent speed within a source can be caused by (1) different patterns of jet components, such as blobs of energetic plasma or forward, reverse, or stationary shocks; (2) a variable Lorentz factor of the jet flow; or (3) different trajectories of components in the jet. The latter can result from a change in the direction of the jet or in the paths of components if each given knot does not fill the entire cross section of the jet.

The observed flux density of a superluminal component is boosted in the direction of the observer by a factor $\delta^{3+\alpha}$, where

$$
\delta=\left[\Gamma\left(1-\beta \cos \Theta_{0}\right)\right]^{-1}
$$

is the Doppler factor and $\alpha$ is the spectral index $\left(S_{\nu} \propto \nu^{-\alpha}\right)$ of the knot. The flux of a component decreases with distance from the core as the result of radiative energy losses and expansion (adiabatic cooling). The emergence of a new component is associated with one or more flares in the radio light curves (e.g., Savolainen et al. 2002). The flare usually has a sharp peak and nearly symmetric exponential rise and decay at high frequencies (Teräsranta \& Valtaoja 1994). In the case of the shock-injet model for the appearance of superluminal knots, variations in flux, spectral energy distribution, and polarization (Marscher \& 

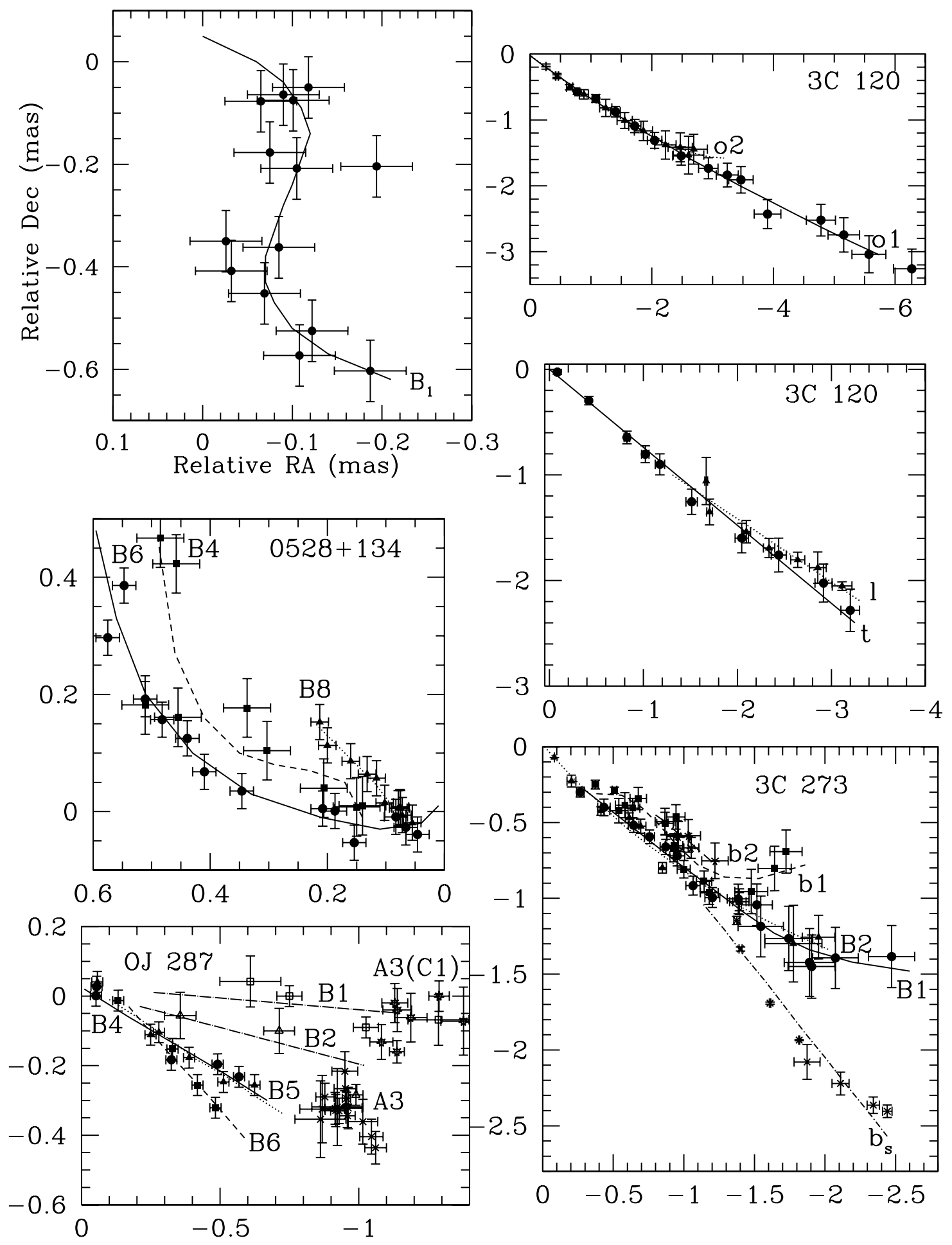

FIG. 18.-Trajectories of components that exhibit a statistically significant change of apparent speed (Table 6). The symbols show the measured positions of the components, while the curves represent polynomial approximations to the trajectories. Ballistic trajectories are shown for 3C 120 (components 1 and t), OJ 287, and CTA 102. (See the Appendix for an explanation.) 

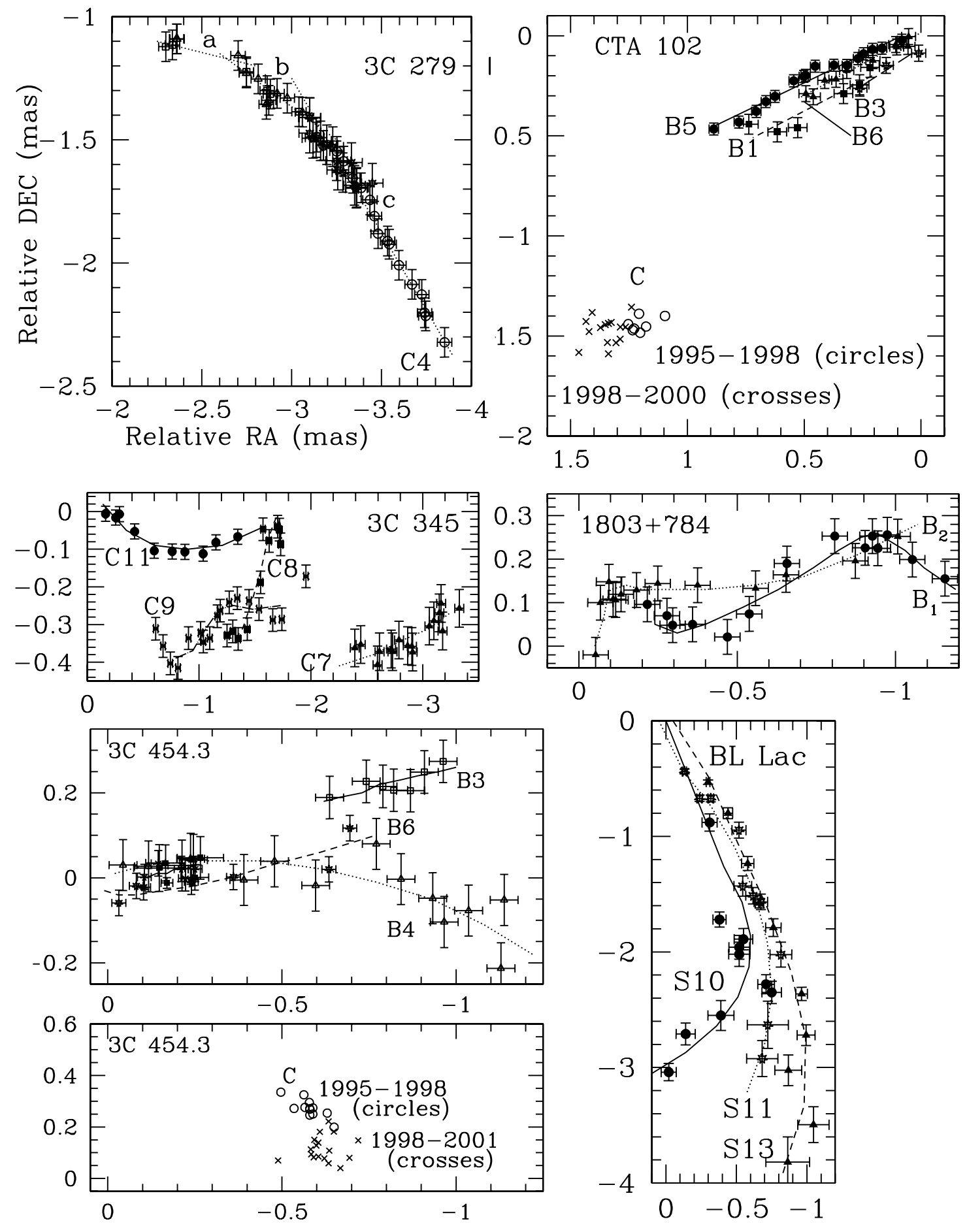

FIG. 18.-Continued 

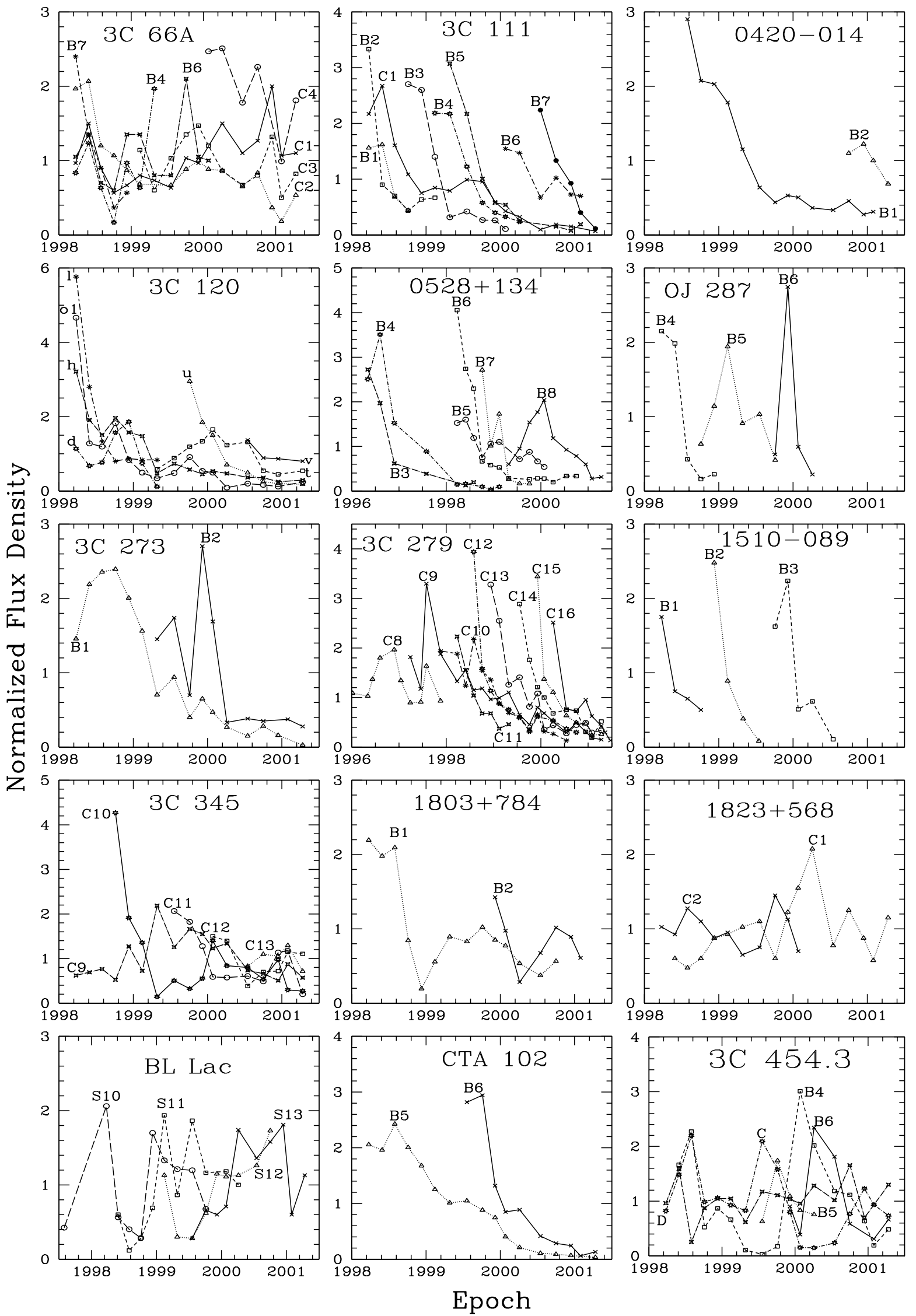

FIG. 19.- - Light curves of superluminal jet components. Flux densities are normalized by the average flux density listed in Table 5 . For each source different components are denoted by different symbols. 


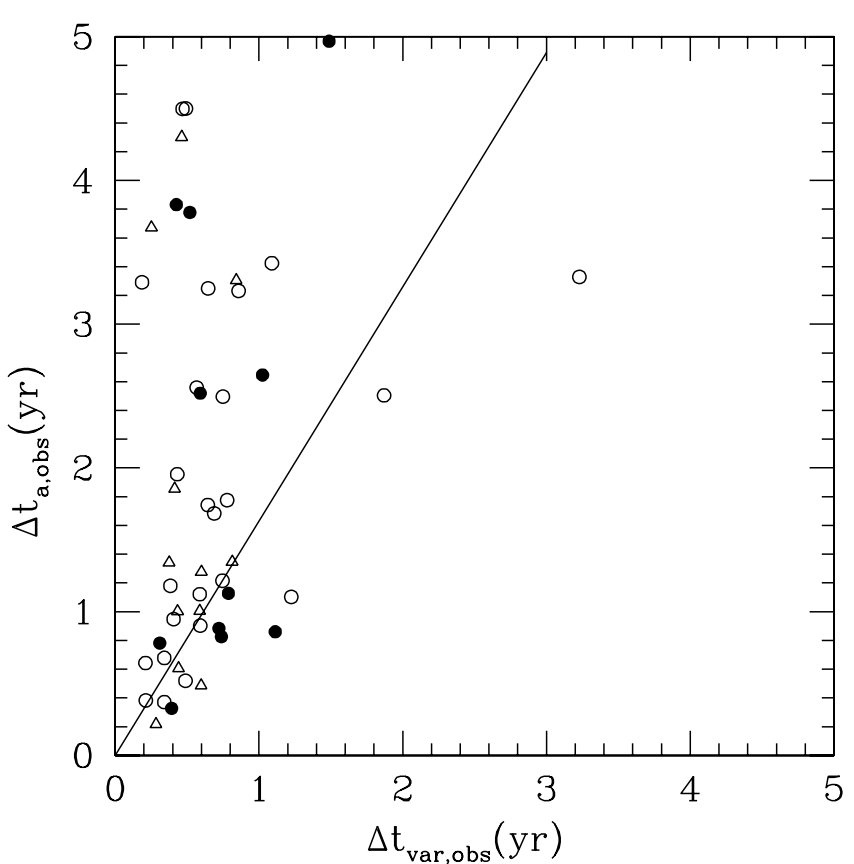

Fig. 20.- Size variability timescale $\Delta t_{a, 0 b s}$ vs. flux variability timescale $\Delta t_{\mathrm{var}, \text { obs }}$ for superluminal components in the quasars (open circles), BL Lac objects ( filled circles), and radio galaxies (triangles). These symbols are applied for all subsequent figures in which radio galaxies, BL Lac objects, and quasars are plotted. The solid line shows the expected relationship during the adiabatic stage defined by Marscher \& Gear (1985).

Gear 1985; Hughes et al. 1985), the symmetric light curves of flares suggest that the flux variability is controlled by light-travel delays across the shocked region (see Sokolov et al. 2004). This assumption allows us to calculate the Doppler factor for each superluminal component using its flux density variability and size measured from the VLBA data. We can then use the derived Doppler factors to study the physical parameters of the jets in our sample.

\subsection{Physical Parameters of the Jet Components}

Figure 19 shows light curves of all superluminal components listed in Table 5 (except knots classified as trailing features; see $\S 6.6)$. Each light curve is normalized by the corresponding average flux indicated in Table 5. We define the timescale of the variability for each superluminal component as $\Delta t_{\mathrm{var}}=$ $d t / \ln \left(S_{\max } / S_{\min }\right)$ (Burbidge et al. 1974), where $S_{\max }$ and $S_{\min }$ are the measured maximum and minimum flux densities, respectively, and $d t$ is the time in years between $S_{\max }$ and $S_{\min }$. The variability Doppler factors are derived as

$$
\delta_{\mathrm{var}}=\frac{s D}{c \Delta t_{\mathrm{var}}(1+z)},
$$

where $D$ is the luminosity distance, $s$ is the angular size of the component, equal to $1.6 a$ for a Gaussian with $\mathrm{FWHM}=a$ measured at the epoch of maximum flux if the true geometry is similar to a uniform face-on disk. In the case of a pointlike knot, we adopt $a=0.1$ mas, which yields an upper limit to $\delta_{\text {var }}$. This definition of the Doppler factor assumes that the variability timescale corresponds to the light-travel time across the knot. This will be true if the radiative cooling time is shorter than the light crossing time, which in turn is shorter than the timescale for cooling by adiabatic expansion. We can roughly verify this assumption by using the relation between flux density and size

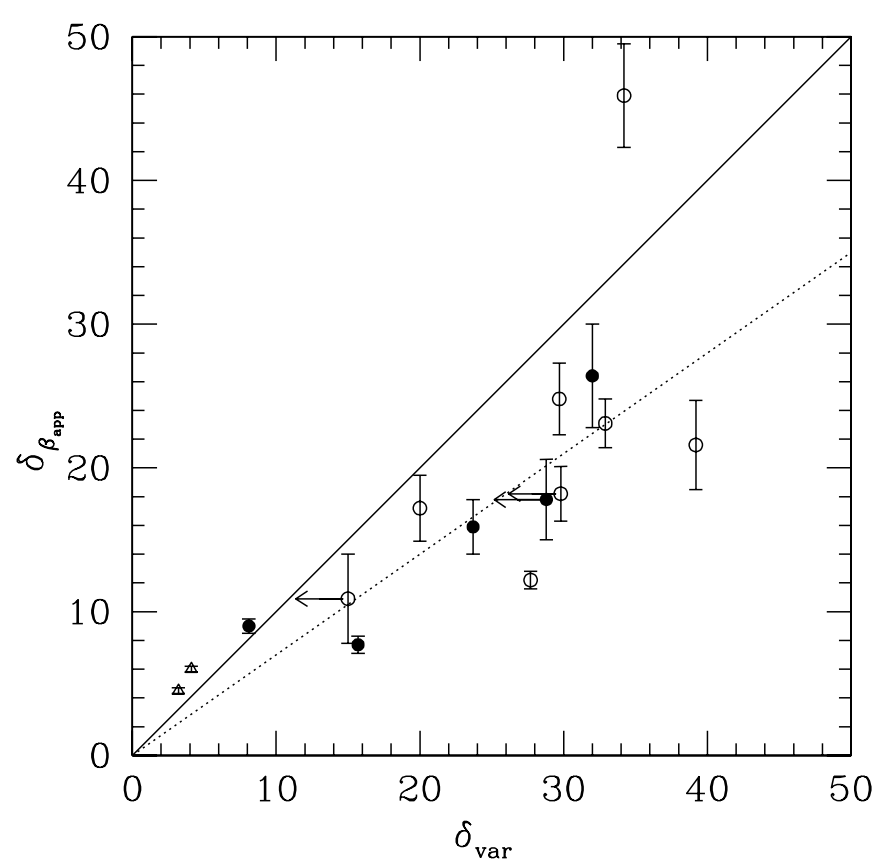

FIG. 21.-Doppler factor $\delta_{\beta_{\text {app }}}$, estimated from the apparent speed for components with the highest apparent speed in the jets, vs. Doppler factor $\delta_{\text {var }}$, computed from the flux variability and size of the components. The solid line shows the relation $\delta_{\beta_{\mathrm{app}}}=\delta_{\mathrm{var}}$, and the dotted line shows the least-squares fit of the observed dependence.

of a shocked region derived in Marscher \& Gear (1985) during the adiabatic stage. We calculate the timescale, $\Delta t_{a}$, of the variability in size for each non-pointlike component as $\Delta t_{a}=$ $d t / \ln \left(a_{\max } / a_{\min }\right)$ (we use $a_{\max }=a_{S_{\min }}$ and $a_{\min }=a_{S_{\max }}$ if $a_{S_{\min }}>$ $a_{S_{\max }}$, which is valid for the majority of components). Figure 20 plots the size variability timescale versus the flux variability timescale. The line indicates the expected relation between $\Delta t_{a}$ and $\Delta t_{\text {var }}$ for adiabatic losses for optically thin shocked gas with $\alpha=0.7$ (Marscher \& Gear 1985). Figure 20 shows that the majority of components have shorter flux variability timescales than those predicted for adiabatic expansion. This implies that at high radio frequencies the decay in flux is driven by radiative losses and therefore that equation (5) should apply to our data.

A widely used method to estimate the Doppler factor from VLBI data assumes that the highest apparent speed detected in a source defines the lowest possible Lorentz factor of the jet, $\Gamma \geq\left(1+\beta_{\text {app }}^{2}\right)^{1 / 2}$. The viewing angle is then taken to be $\Theta_{0} \leq$ $\sin ^{-1}\left(1 / \beta_{\text {app }}\right)$; this leads to $\delta_{\beta_{\text {app }}} \sim \beta_{\text {app }}$. In Figure 21 Doppler factors thus derived are plotted versus the Doppler factor computed from the flux variability and size of the components that have the highest apparent speed in the jets. Figure 21 demonstrates that there is reasonable agreement between the Doppler factors estimated by these two different methods. The best least-squares linear fit to the dependence is $\delta_{\beta_{\text {app }}}=(0.72 \pm 0.15) \delta_{\text {var }}$, consistent with $\delta_{\text {var }}$ being the true value and $\delta_{\text {app }}$ being a lower limit, taking into account that $\delta_{\beta_{\text {app }}}$ is estimated for the lowest possible Lorentz factor. We conclude that the combination of variability of flux and measurement of angular sizes provides a new, robust method for deriving Doppler factors from well-sampled sequences of VLBI data.

Tables 8, 9, and 10 give estimates of the Lorentz factor, viewing angle, Doppler factor, observed brightness temperature $\left(T_{b, \text { obs }}\right)$, and intrinsic brightness temperature $\left(T_{b}\right.$,int $)$ for 43 knots in the quasars, 19 knots in the BL Lac objects, and 15 knots in the radio galaxies, respectively. The Lorentz factor and viewing angle are 
TABLE 8

Jet Components in Quasars

\begin{tabular}{|c|c|c|c|c|c|c|}
\hline Source & Knot & $\Gamma^{\mathrm{var}}$ & $\begin{array}{c}\Theta_{0}^{\mathrm{var}} \\
(\mathrm{deg})\end{array}$ & $\delta^{\mathrm{var}}$ & $\begin{array}{c}T_{b, \text { obs }} \\
\left(10^{10} \mathrm{~K}\right)\end{array}$ & $\begin{array}{c}T_{b, \text { int }} \\
\left(10^{9} \mathrm{~K}\right)\end{array}$ \\
\hline \multirow[t]{2}{*}{$0420-014 \ldots \ldots \ldots \ldots$} & B2 & 10.4 & 2.4 & $<17.5$ & 4.69 & 1.09 \\
\hline & B1 & 11.5 & 3.6 & $<15.0$ & 1.64 & 0.49 \\
\hline \multirow[t]{6}{*}{$0528+134 \ldots \ldots \ldots \ldots \ldots$} & B8 & 27.4 & 0.4 & $<53.2$ & 7.94 & 0.62 \\
\hline & B7 & 35.2 & 0.5 & $<63.6$ & 2.17 & 0.125 \\
\hline & B6 & 25.5 & 1.2 & 39.2 & 2.58 & 0.34 \\
\hline & B5 & 21.1 & 3.3 & 17.3 & 0.38 & 0.20 \\
\hline & B4 & 27.5 & 0.8 & 48.5 & 3.72 & 0.34 \\
\hline & B3 & 39.5 & 0.6 & 69.2 & 1.07 & 0.05 \\
\hline \multirow[t]{3}{*}{ 3C $273 \ldots \ldots \ldots \ldots \ldots . .}$. & B2 & 13.8 & 6.9 & 7.4 & 15.4 & 6.62 \\
\hline & B1 & 8.3 & 6.1 & 9.4 & 16.4 & 4.66 \\
\hline & $\mathrm{C} 1$ & 8.2 & 3.8 & 12.6 & 0.23 & 0.04 \\
\hline \multirow[t]{10}{*}{$3 \mathrm{C} 279$} & $\mathrm{C} 16$ & 17.1 & 2.9 & 19.7 & 20.2 & 2.65 \\
\hline & $\mathrm{C} 15$ & 18.2 & 2.3 & 23.9 & 25.3 & 2.38 \\
\hline & $\mathrm{C} 14$ & 17.4 & 2.8 & 20.0 & 9.37 & 1.19 \\
\hline & $\mathrm{C} 13$ & 16.3 & 3.3 & 17.6 & 16.1 & 2.57 \\
\hline & $\mathrm{C} 12$ & 16.6 & 2.5 & 22.0 & 23.3 & 2.52 \\
\hline & $\mathrm{C} 11$ & 12.9 & 2.2 & $<20.9$ & 12.6 & 1.49 \\
\hline & $\mathrm{C} 10$ & 14.9 & 1.7 & 24.7 & 4.96 & 0.44 \\
\hline & C9 & 16.7 & 1.6 & 27.3 & 3.74 & 0.28 \\
\hline & $\mathrm{C} 8$ & 17.2 & 0.6 & 33.4 & 6.47 & 0.35 \\
\hline & $\mathrm{C} 4$ & 10.5 & 3.9 & 13.8 & 1.05 & 0.25 \\
\hline \multirow[t]{3}{*}{$1510-089$............. } & B3 & 30.1 & 1.3 & 41.3 & 1.10 & 0.03 \\
\hline & B2 & 35.2 & 1.5 & 39.0 & 0.73 & 0.02 \\
\hline & B1 & 47.9 & 1.6 & 34.2 & 0.21 & 0.01 \\
\hline \multirow[t]{7}{*}{ 3C $345 \ldots \ldots \ldots \ldots \ldots \ldots$} & $\mathrm{C} 13$ & 16.2 & 2.3 & 23.1 & 7.41 & 0.79 \\
\hline & $\mathrm{C} 12$ & 24.6 & 1.6 & 32.9 & 3.25 & 0.19 \\
\hline & $\mathrm{C} 11$ & 29.8 & 3.8 & 12.0 & 7.37 & 2.39 \\
\hline & $\mathrm{C} 10$ & 19.3 & 3.3 & 17.6 & 12.1 & 2.05 \\
\hline & C9 & 16.2 & 4.6 & 11.9 & 1.51 & 0.49 \\
\hline & $\mathrm{C} 8$ & 19.9 & 0.9 & 36.5 & 0.50 & 0.025 \\
\hline & $\mathrm{C} 7$ & 13.3 & 2.0 & 21.9 & 0.02 & 0.003 \\
\hline \multirow[t]{2}{*}{ CTA $102 \ldots \ldots \ldots \ldots . . .}$. & B6 & 20.5 & 1.7 & $<29.8$ & 3.54 & 0.37 \\
\hline & B5 & 15.7 & 3.0 & 18.8 & 14.4 & 3.29 \\
\hline \multirow[t]{6}{*}{ 3C $454.3 \ldots \ldots \ldots \ldots \ldots$} & B6 & 25.2 & 1.9 & 29.7 & 17.6 & 1.58 \\
\hline & B5 & 14.8 & 1.5 & $<25.8$ & 1.58 & 0.18 \\
\hline & B4 & 20.0 & 3.9 & 13.9 & 4.54 & 1.49 \\
\hline & B3 & 13.4 & 3.7 & 15.4 & 24.7 & 6.76 \\
\hline & $\mathrm{C}$ & 14.2 & 0.2 & 28.2 & 6.76 & 0.66 \\
\hline & $\mathrm{D}$ & 10.2 & 1.7 & 18.7 & 0.03 & 0.007 \\
\hline
\end{tabular}

solutions of the system that combine equations (3) and (4) for a knot having a measured apparent speed and a Doppler factor derived via equation (5). The observed brightness temperature is computed from the VLBI measurements as $T_{b \text {.obs }}=7.5 \times$ $10^{8} S_{\max } / s^{2} \mathrm{~K}$, where $S_{\max }$ is the maximum flux of the component in janskys and $s$ is its measured angular size in milliarcseconds at the epoch of maximum flux (see above). We apply the Doppler factors derived with equation (5) to estimate the intrinsic brightness temperature, $T_{b \text {,int }}=T_{b, \text { obs }}(1+z)^{1.7} / \delta^{1.7}$, where we adopt $\alpha=0.7$.

\subsection{Opening Angle of the Jet}

We assume that jets have conical structure and a constant angle $\theta$ between the jet axis and surface of the cone that contains the entire region of emission. Therefore, $\theta$ is the actual half opening angle of the jet. This assumption is likely correct for the section of the jet within 1-2 mas from the core, while at larger distances $(\geq 10$ mas $)$ the jet could trace out a helical structure covering a wide range of position angles even if the body of the jet remains narrow (e.g., Lister et al. 2003). We estimate the projected half opening angle, $\theta_{p}$, for each source using the ratio
TABLE 9

Jet Components in BL Lac OBjects

\begin{tabular}{|c|c|c|c|c|c|c|}
\hline Source & Knot & $\Gamma^{\mathrm{var}}$ & $\begin{array}{c}\Theta_{0}^{\mathrm{var}} \\
(\mathrm{deg})\end{array}$ & $\delta^{\mathrm{var}}$ & $\begin{array}{c}T_{b, \text { obs }} \\
\left(10^{10} \mathrm{~K}\right)\end{array}$ & $\begin{array}{c}T_{b, \text { int }} \\
\left(10^{9} \mathrm{~K}\right)\end{array}$ \\
\hline \multirow[t]{7}{*}{ 3C 66A................... } & $\mathrm{C} 4$ & 5.0 & 1.8 & $<9.7$ & 0.73 & 0.285 \\
\hline & C3 & 5.9 & 2.8 & $<10.9$ & 0.44 & 0.141 \\
\hline & $\mathrm{C} 2$ & 7.7 & 2.8 & $<13.5$ & 0.35 & 0.079 \\
\hline & $\mathrm{C} 1$ & 11.4 & 0.8 & 22.2 & 0.15 & 0.014 \\
\hline & B7 & 29.7 & 3.3 & $<15.4$ & 0.21 & 0.037 \\
\hline & B6 & 26.1 & 4.0 & 12.1 & 0.12 & 0.032 \\
\hline & B4 & 27.6 & 2.5 & 22.1 & 0.02 & 0.002 \\
\hline \multirow[t]{3}{*}{ OJ $287 \ldots \ldots \ldots \ldots \ldots \ldots$} & B6 & 20.7 & 4.7 & 10.6 & 0.41 & 0.117 \\
\hline & B5 & 12.3 & 3.4 & 16.1 & 1.31 & 0.183 \\
\hline & B4 & 19.9 & 1.8 & $<28.8$ & 1.70 & 0.088 \\
\hline \multirow[t]{3}{*}{$1803+784 \ldots \ldots \ldots \ldots \ldots$} & B2 & 17.2 & 2.2 & 23.7 & 0.34 & 0.038 \\
\hline & B1 & 15.7 & 3.6 & 16.1 & 0.25 & 0.054 \\
\hline & $\mathrm{C} 1$ & 20.8 & 0.8 & 38.7 & 0.02 & 0.001 \\
\hline \multirow[t]{2}{*}{$1823+568 \ldots \ldots \ldots \ldots \ldots$} & $\mathrm{C} 2$ & 9.8 & 2.9 & 15.7 & 0.20 & 0.005 \\
\hline & $\mathrm{C} 1$ & 13.8 & 0.6 & 27.0 & 0.14 & 0.012 \\
\hline \multirow[t]{4}{*}{ BL Lac ................... } & S13 & 9.1 & 7.0 & 8.1 & 0.03 & 0.009 \\
\hline & $\mathrm{S} 12$ & 8.6 & 7.9 & 7.2 & 0.16 & 0.061 \\
\hline & S11 & 7.2 & 13.2 & 3.9 & 0.07 & 0.082 \\
\hline & S10 & 5.1 & 6.0 & 8.0 & 0.07 & 0.025 \\
\hline
\end{tabular}

between apparent transverse size, $s_{t}$, of the jet and apparent longitudinal distance, $s_{l}$, of the components: $\theta_{p}=\tan ^{-1} \psi$, where $\psi$ is the slope of the best linear fit to the relation between $s_{t}$ and $s_{l}$ as defined at the position of each component that is brighter than $1 \%$ of the peak intensity. The values of $s_{t}$ and $s_{l}$ are calculated as follows: $s_{l}=R$, where $R$ is the observed separation of the component from the core; and $s_{t}=R \sin \left(\left|\Theta_{\text {jet }}-\Theta\right|\right)+a / 2$, where $\Theta_{\text {jet }}$ is the projected direction of the jet as defined by the mean position angle of all sufficiently bright components over all epochs, $\Theta$ is the position angle of the component, and $a$ is the size of the component. We first plot the values of $s_{t}$ against $s_{l}$ to estimate a preliminary linear dependence. The preliminary relationship is used to remove points that deviate from the dependence by more than $3 \sigma$ of the linear model. Then the value of $\psi$ is obtained by minimizing $\chi^{2}$ of the adjusted data. In Figure 22 all pairs of $\left(s_{t}, s_{l}\right)$ are plotted, and the best linear fit of the adjusted data is presented for each source. Figure 22 shows that a jet model with a constant opening angle provides a good approximation to the inner jet, although the plots of two BL Lac objects

TABLE 10

Jet Components in Radio Galaxies

\begin{tabular}{|c|c|c|c|c|c|c|}
\hline Source & Knot & $\Gamma^{\mathrm{var}}$ & $\begin{array}{c}\Theta_{0}^{\text {var }} \\
\text { (deg) }\end{array}$ & $\delta^{\mathrm{var}}$ & $\begin{array}{c}T_{b, \text { obs }} \\
\left(10^{10} \mathrm{~K}\right)\end{array}$ & $\begin{array}{c}T_{b, \text { int }} \\
\left(10^{9} \mathrm{~K}\right)\end{array}$ \\
\hline \multirow[t]{8}{*}{ 3C $111 \ldots \ldots \ldots \ldots \ldots$} & B7 & 2.8 & 24.8 & 2.3 & 1.49 & 3.82 \\
\hline & B6 & 3.7 & 22.9 & 2.4 & 8.00 & 0.20 \\
\hline & B5 & 3.5 & 19.2 & $<3.0$ & 1.44 & 2.40 \\
\hline & B4 & 4.0 & 24.4 & 2.1 & 1.61 & 4.94 \\
\hline & B3 & 4.8 & 10.4 & $<5.5$ & 0.06 & 0.04 \\
\hline & B2 & 4.2 & 21.6 & 2.5 & 0.29 & 0.69 \\
\hline & B1 & 3.2 & 12.5 & $<4.3$ & 0.23 & 0.21 \\
\hline & $\mathrm{C} 1$ & 6.6 & 13.0 & 4.1 & 0.15 & 0.15 \\
\hline \multirow[t]{7}{*}{ 3C $120 \ldots \ldots \ldots \ldots \ldots$} & $\mathrm{v}$ & 6.6 & 23.0 & 1.7 & 0.19 & 0.85 \\
\hline & $\mathrm{u}$ & 4.3 & 22.3 & 2.3 & 0.75 & 1.89 \\
\hline & $\mathrm{t}$ & 4.9 & 16.9 & 3.2 & 0.11 & 0.16 \\
\hline & ol & 7.0 & 22.0 & 1.7 & 3.69 & 15.2 \\
\hline & 1 & 4.0 & 21.4 & 2.5 & 0.88 & 1.91 \\
\hline & $\mathrm{h}$ & 4.5 & 19.8 & 2.7 & 0.31 & 0.61 \\
\hline & $\mathrm{d}$ & 3.9 & 11.3 & 4.9 & 0.03 & 0.02 \\
\hline
\end{tabular}




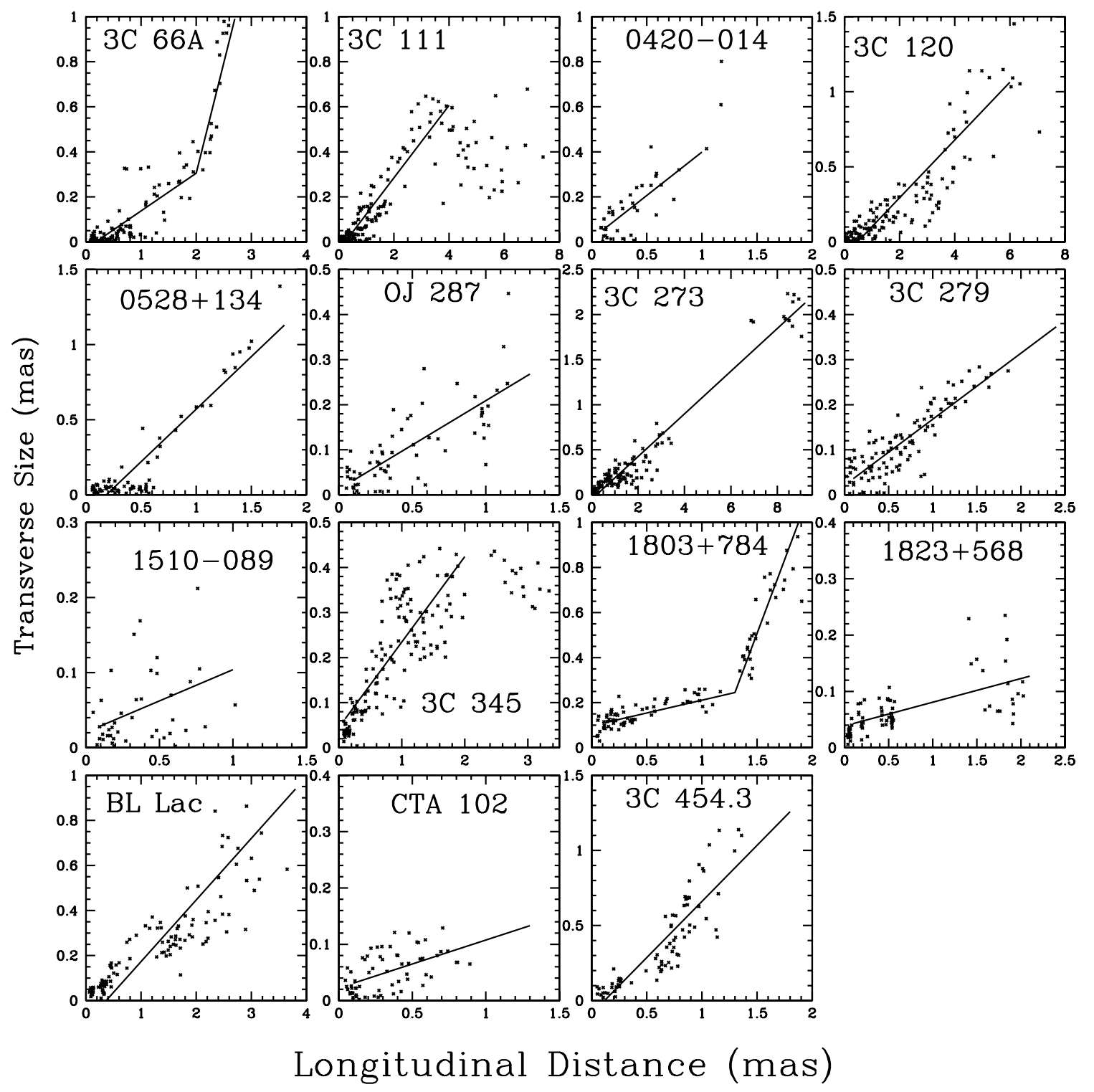

FIG. 22.-Projected transverse size of the jet vs. longitudinal distance from the core. The solid lines represent the best linear fit to the data.

(3C 66A and 1803+784) display an increase in $\theta_{p}$ beyond 2 and 1 mas, respectively, which might be a general feature of many BL Lac jets. In the radio galaxy 3C 111 and quasar 3C 345 a decrease in $\theta_{p}$ is observed and is most likely caused by the weakness and diffuse nature of components at large distances from the core, such that our images only contain portions of these features. Table 11 gives the parameters $\Theta_{\text {jet }}, \theta_{p},\left\langle\Theta_{0}\right\rangle, \theta,\langle\Gamma\rangle$, and $\langle\delta\rangle$ for each source. Parameters $\langle\Gamma\rangle,\langle\delta\rangle$, and $\left\langle\Theta_{0}\right\rangle$ are weighted averages of the values for the individual components listed in Tables $8_{-}^{-}$ 10 , with weights inversely proportional to the uncertainty in apparent speed. The intrinsic half opening angle, $\theta$, is estimated as $\theta=\theta_{p} \sin \left\langle\Theta_{0}\right\rangle$, where $\left\langle\Theta_{0}\right\rangle$ is the angle between the jet axis and the line of sight.

\subsection{Jet Parameters}

We have constructed the dependence between the Doppler beaming factor $\left(\delta_{\mathrm{var}}\right)$ and apparent speed (Fig. 23) to compare derived parameters with results from the $2 \mathrm{~cm}$ VLBA survey (Kellermann et al. 2004). These authors determined Doppler factors using the variability method of Lähteenmäki \& Valtaoja (1999), with an intrinsic brightness temperature of $2 \times 10^{10} \mathrm{~K}$, which they assume corresponds to the region of the VLBI core where the main variability at radio frequencies occurs (Savolainen et al. 2002). In Figure 23 the majority of points follow the expectation that $\beta_{\text {app }} \lesssim \delta_{\text {var }}$ and lie inside the " $1 / \beta_{\text {app }}$ cone." Moreover, despite our sample consisting of blazars or blazar-like sources for which a higher Lorentz factor is expected, the upper limit to $\beta_{\text {app }}$ corresponding to $\Gamma=25$ (Kellermann et al. 2004) applies to our sample as well (Fig. 23, dotted curve). Figure 24 shows the distribution of Lorentz factors of superluminal components. There is a significant scatter in $\Gamma$ for the quasars and BL Lac objects, and the Lorentz factors of the radio galaxies are lower than those of the other classes. The distributions, in general, agree with the distribution of Lorentz factors found in the $2 \mathrm{~cm}$ survey.

Monte Carlo simulations for flux-limited samples of radio sources predict a connection, although not a direct correlation, between the Lorentz factor and viewing angle of each jet: the higher the Lorentz factor, the smaller the viewing angle (Lister $\&$ Marscher 1997). Our sample of the blazars and blazar-like sources shows a significant correlation between $1 / \Gamma$ and $\Theta_{0}$ (coefficient of correlation 0.83 , Fig. 25); this supports the above 
TABLE 11

Global Parameters of the Jets

\begin{tabular}{|c|c|c|c|c|c|c|c|c|}
\hline Source & $\begin{array}{c}D \\
(\mathrm{Gpc})\end{array}$ & $\begin{array}{c}\Theta_{\text {jet }} \\
(\mathrm{deg})\end{array}$ & $\begin{array}{c}\theta_{p} \\
(\mathrm{deg})\end{array}$ & $\begin{array}{c}\left\langle\Theta_{0}\right\rangle \\
(\mathrm{deg})\end{array}$ & $\begin{array}{c}\theta \\
(\mathrm{deg})\end{array}$ & $\langle\Gamma\rangle$ & $\langle\delta\rangle$ & $f_{\mathrm{ej}}$ \\
\hline 3C 66A... & 2.46 & $-162.0 \pm 25$ & $9.5 \pm 4.6$ & $2.1 \pm 1.0$ & $0.4 \pm 0.2$ & $10.4 \pm 3.5$ & $16.0 \pm 4.9$ & 1.9 \\
\hline ....................... & 0.222 & $63.5 \pm 4.5$ & $9.1 \pm 2.7$ & $18.1 \pm 5.0$ & $2.8 \pm 0.8$ & $4.4 \pm 1.3$ & $3.4 \pm 1.1$ & 2.4 \\
\hline $0420-014 \ldots \ldots \ldots \ldots \ldots \ldots \ldots \ldots$ & 5.925 & $-139.2 \pm 21.9$ & $21 \pm 10$ & $3.0 \pm 0.6$ & $1.1 \pm 0.5$ & $11.0 \pm 0.5$ & $16.2 \pm 1.2$ & 1.1 \\
\hline 3C $120 \ldots \ldots \ldots$ & 0.145 & $-121.7 \pm 6.1$ & $10.9 \pm 3.8$ & $20.5 \pm 1.8$ & $3.8 \pm 1.3$ & $5.3 \pm 1.2$ & $2.4 \pm 0.6$ & 2.4 \\
\hline $0528+134 \ldots \ldots \ldots \ldots \ldots \ldots$ & 16.12 & $74.7 \pm 22.4$ & $35.1 \pm 9.3$ & $1.1 \pm 1.0$ & $0.7 \pm 0.2$ & $28.3 \pm 4.5$ & $47.5 \pm 13.8$ & 7.0 \\
\hline OJ $287 \ldots \ldots \ldots$ & 1.59 & $-105.8 \pm 16.9$ & $11.1 \pm 5.4$ & $3.2 \pm 0.9$ & $0.8 \pm 0.4$ & $16.5 \pm 4.0$ & $18.9 \pm 6.4$ & 1.3 \\
\hline 3C 273 & 0.76 & $-126.4 \pm 8.6$ & $13.4 \pm 2.5$ & $6.1 \pm 0.8$ & $1.4 \pm 0.3$ & $10.6 \pm 2.8$ & $9.0 \pm 1.4$ & 0.7 \\
\hline 3C 279 & 3.10 & $-131.5 \pm 10.8$ & $8.3 \pm 2.7$ & $2.1 \pm 1.1$ & $0.4 \pm 0.2$ & $15.5 \pm 2.5$ & $24.1 \pm 6.5$ & 3.5 \\
\hline $1510-089$ & 1.93 & $-42.6 \pm 16.4$ & $4.8 \pm 6.5$ & $1.4 \pm 0.4$ & $0.2 \pm 0.2$ & $36.6 \pm 7.0$ & $38.6 \pm 2.8$ & 2.6 \\
\hline 3C 345 & 3.50 & $-94.9 \pm 8.6$ & $10.7 \pm 4.4$ & $2.7 \pm 0.9$ & $0.5 \pm 0.2$ & $18.7 \pm 5.5$ & $20.2 \pm 5.0$ & 3.7 \\
\hline $1803+784 \ldots \ldots \ldots \ldots \ldots \ldots$ & 4.11 & $-78.6 \pm 31.1$ & $7.5 \pm 3.6$ & $2.1 \pm 1.2$ & $0.3 \pm 0.2$ & $18.1 \pm 2.3$ & $27.0 \pm 9.9$ & 1.0 \\
\hline $1823+568 \ldots \ldots \ldots \ldots \ldots \ldots \ldots$ & 4.00 & $-162.6 \pm 5.9$ & $2.4 \pm 2.0$ & $1.6 \pm 1.1$ & $0.1 \pm 0.2$ & $12.0 \pm 2.0$ & $21.9 \pm 5.6$ & 0.5 \\
\hline 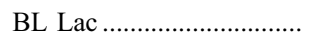 & 0.31 & $-161.1 \pm 7.3$ & $13.9 \pm 4.3$ & $7.7 \pm 1.9$ & $1.9 \pm 0.6$ & $7.0 \pm 1.8$ & $7.2 \pm 1.1$ & 1.1 \\
\hline CTA $102 \ldots \ldots \ldots$ & 6.92 & $123.1 \pm 19.9$ & $4.8 \pm 1.9$ & $2.6 \pm 0.5$ & $0.2 \pm 0.1$ & $17.2 \pm 2.0$ & $22.3 \pm 4.5$ & 1.2 \\
\hline 3C $454.3 \ldots \ldots \ldots \ldots \ldots \ldots \ldots$ & 5.48 & $-76.8 \pm 27.8$ & $36.8 \pm 9.8$ & $1.3 \pm 1.2$ & $0.8 \pm 0.2$ & $15.6 \pm 2.2$ & $24.6 \pm 4.5$ & 1.9 \\
\hline
\end{tabular}

prediction. Small intrinsic bends in jets oriented very close to the line of sight should be greatly amplified in projection on the sky (Readhead et al. 1978). The expected morphology has not been confirmed by VLBI surveys. For example, $\gamma$-ray blazars, which exhibit the highest apparent speeds (Jorstad et al. 2001b; Kellermann et al. 2004), do not possess more pronounced bends than sources not yet detected in $\gamma$-rays (Kellermann et al. 1998; Jorstad et al. 2001b). Estimates of the viewing angle obtained for our sample allow us to test the relation between morphology and viewing angle of the jets more directly. We have determined the average projected position angle of the jet, $\Theta_{\text {jet }}$, and its standard deviation, $\sigma\left(\Theta_{\text {jet }}\right)$, for each source (see Table 11). In sources with jet direction close to the line of sight, a small intrinsic change of the component trajectory should result in a significant bend in the projected path, leading to a large scatter in $\Theta_{\text {jet }}$ and yielding a high value of $\sigma\left(\Theta_{\text {jet }}\right)$. A similar test is to compare the projected opening angle with the viewing angle of the jet: jets viewed very close to the line of sight should have a wide projected opening angle on VLBI maps. Figure 26 presents both plots. The left panel shows the relationship between $\Theta_{0}$ and $\sigma\left(\Theta_{\text {jet }}\right)$. Although there is a general decrease in $\sigma\left(\Theta_{\text {jet }}\right)$ with increasing angle between the jet axis and line of sight (coefficient of correlation - 0.58 ), the

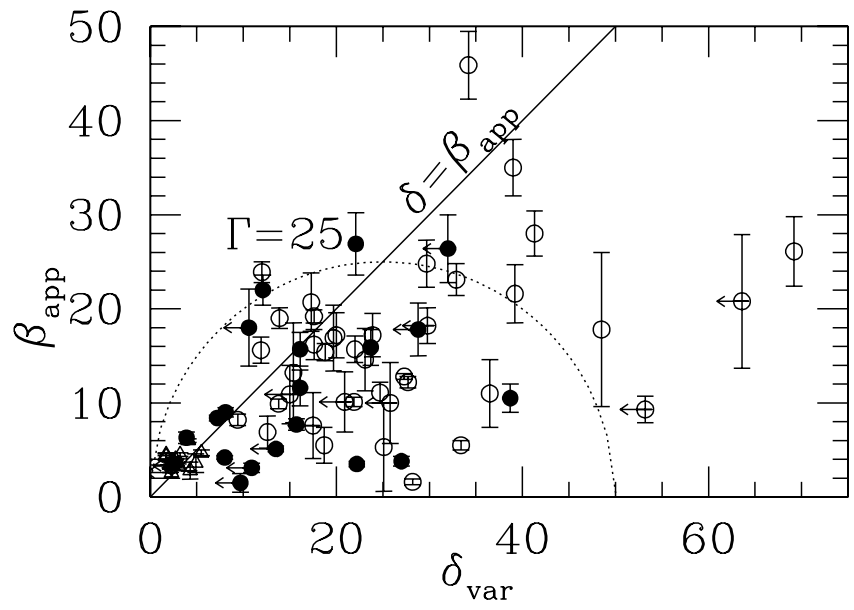

FIG. 23.-Apparent speed vs. variability Doppler factor for superluminal components. The solid line denotes $\delta_{\mathrm{var}}=\beta_{\text {app }}$. The dotted curve encloses the expected location of points corresponding to $\Gamma \leq 25$. relationship is very weak or absent for viewing angles $\lesssim 5^{\circ}$ (coefficient of correlation -0.18 ). The right panel shows that there is no connection between the projected opening angle and viewing angle of the jet (coefficient of correlation -0.16). This result implies that amplification of the projected size by a small angle between the jet axis and line of sight is partly (or sometimes completely) canceled by the proportionality between opening angle and $1 / \Gamma$ (see below). Possible reasons behind the lack of correlation between the degree of apparent bending and the small viewing angle include (1) an inverse relationship between bulk Lorentz factor and degree of intrinsic bending, given that the momentum of the jet is proportional to $\Gamma,(2)$ broader opening angles in slower jets, with filamentary structure appearing similar to bending of the axis, and (3) the available resolution transverse to the jet not being sufficient to detect bending in many high- $\Gamma$ objects.
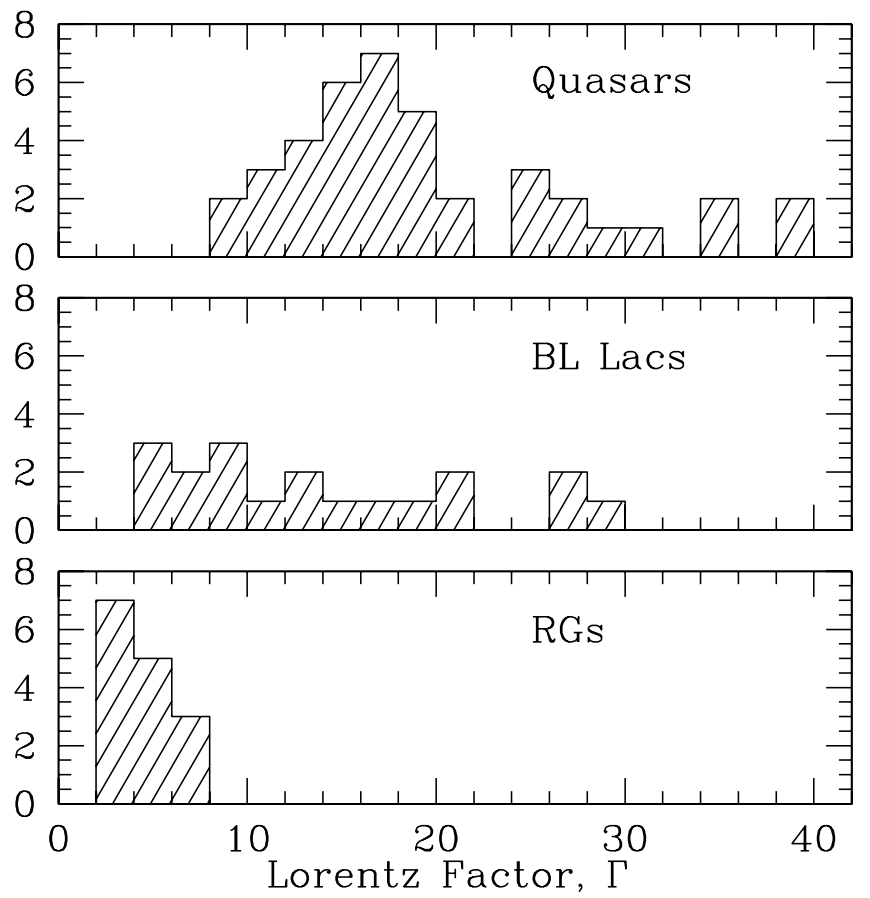

FIG. 24.-Distribution of Lorentz factors of superluminal jet components in the eight quasars, five BL Lac objects, and two radio galaxies. 


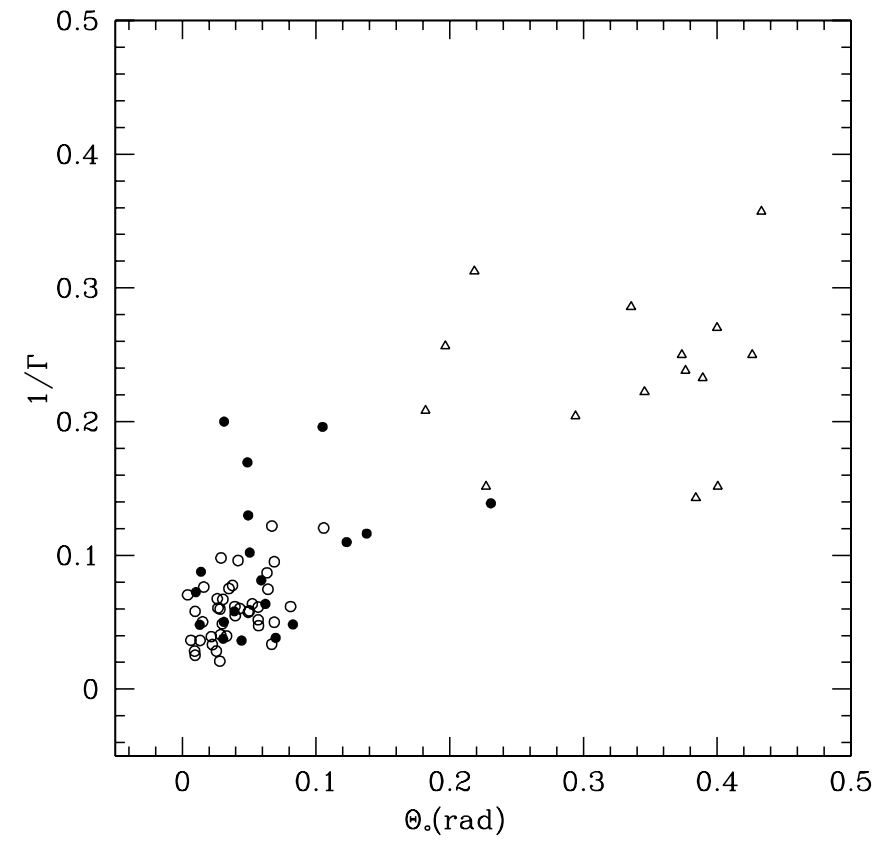

FIG. 25.-Inverse Lorentz factor vs. viewing angle for superluminal jet components.

The Lorentz factor plays a significant role in determining the jet geometry. According to standard models of relativistic jets, the opening angle of the jet should be inversely proportional to the Lorentz factor (e.g., Blandford \& Königl 1979). In Figure 27 the estimated half opening angles are plotted versus the derived Lorentz factors. There is an obvious decrease of $\theta$ toward higher values of $\Gamma$. According to a $\chi^{2}$ test the observed dependence can be described by the relation $\theta \approx \rho / \Gamma \mathrm{rad}$, where $\rho=0.17 \pm 0.08$ (Fig. 27, solid line) at a $2.5 \%$ level of significance $\left(\chi^{2}=6.875\right.$, dof $=2$ ). The current models for the formation of relativistic jets that employ confinement of a jet by magnetic forces (e.g., Meier et al. 2001; Vlahakis \& Königl 2004) do not yet specify the opening angle of the jet as a function of the model parameters. Such

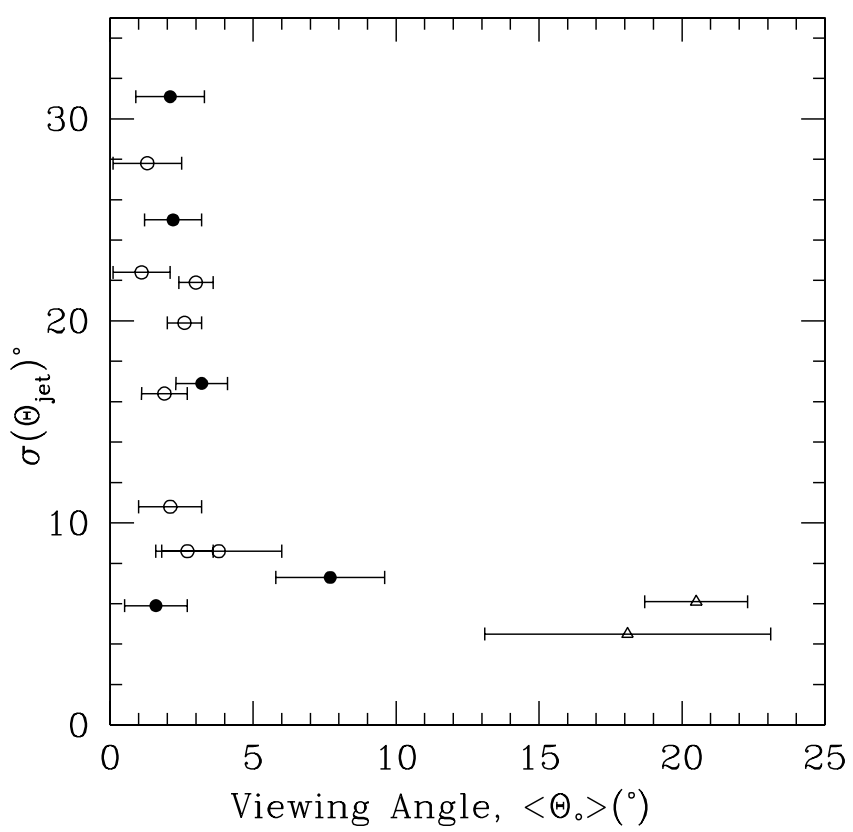

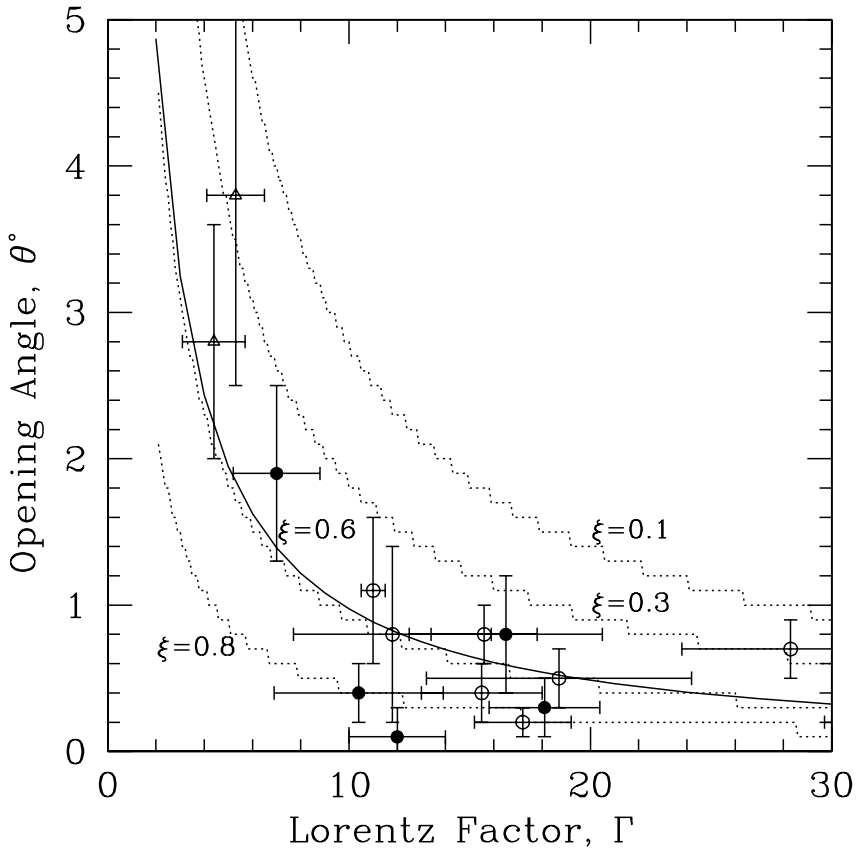

FIG. 27.- Half opening angle vs. Lorentz factor of the jets. The solid curve corresponds to the best approximation according to the $\chi^{2}$ test of the observed dependence within the assumed law $\theta=\rho / \Gamma$, where $\rho$ is a constant. The dotted curves represent relationships between the half opening angle and Lorentz factors expected in the gasdynamical model for different values of the parameter $\xi$.

an expression, however, has been derived based on gas dynamics for a relativistic jet confined by pressure equilibrium with its surroundings (Daly \& Marscher 1988). In this model the opening angle depends on the Lorentz factor of the flow and the ratio of the external pressure, $P_{\text {ext }}$, to the initial pressure, $P_{0}$, of the plasma in the core region, $\xi=\left(P_{\text {ext }} / P_{0}\right)^{1 / 2}$. Using equations (18), (19a), and (19b) in Daly \& Marscher (1988), we calculate the dependence of the opening angle on the Lorentz factor for different values of $\xi$ (Fig. 27, dotted curves). The best fit of the

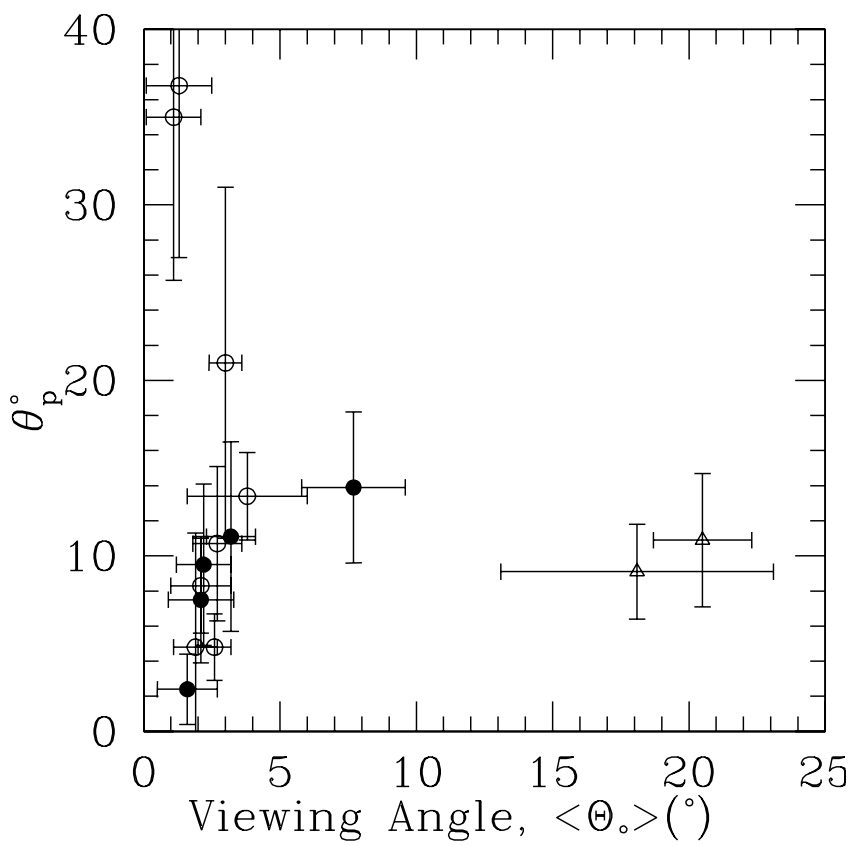

FIG. 26.-Left: Scatter in the projected jet position angle vs. the average viewing angle of the jets. Right: Projected half opening angle vs. average viewing angle. 

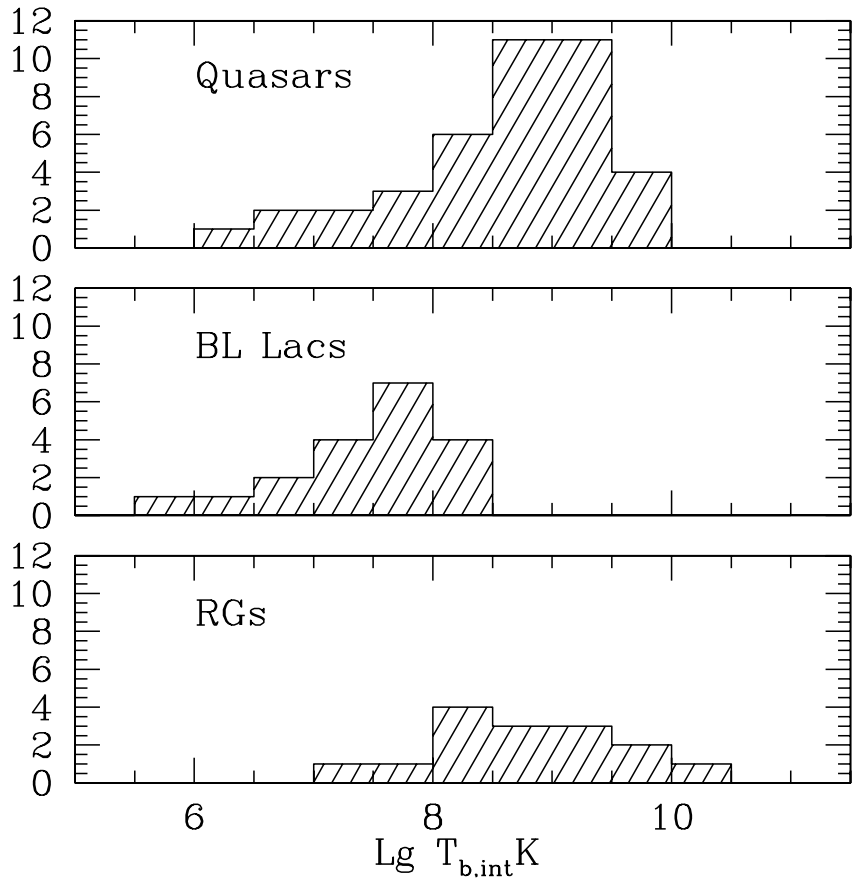

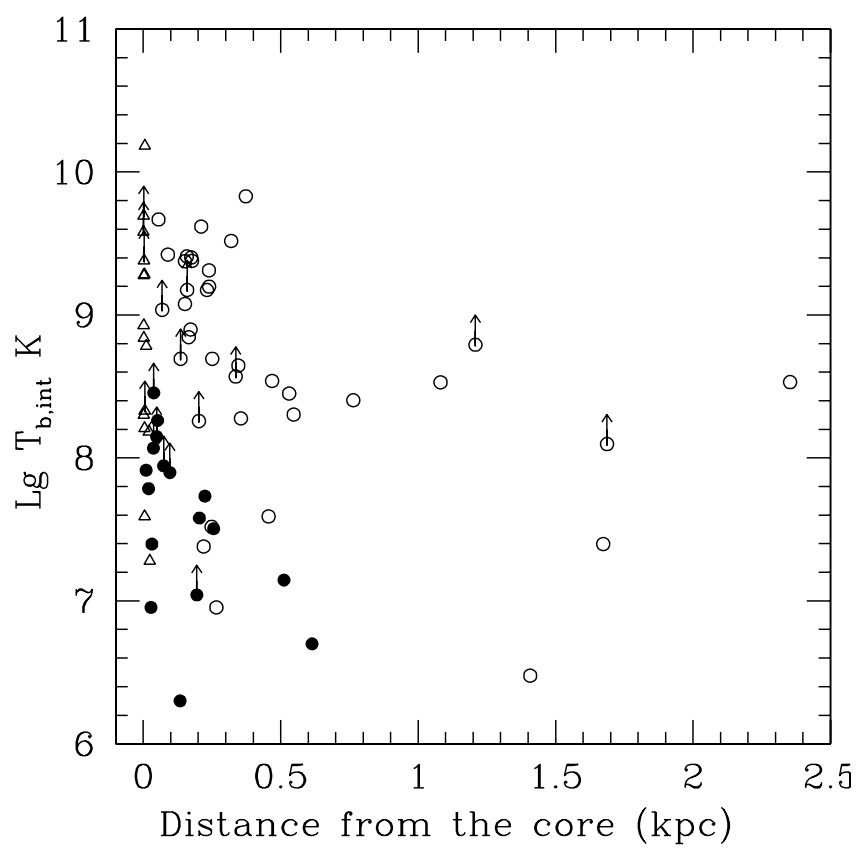

Fig. 28. - Left: Distribution of intrinsic brightness temperature of the superluminal jet components in eight quasars, five BL Lac objects, and two radio galaxies. Right: Intrinsic brightness temperature vs. deprojected distance from the core

observed dependence ( $5 \%$ level of significance) coincides with the model in which $\xi=0.6\left(\chi^{2}=4.586\right.$, dof $\left.=2\right)$, corresponding to a pressure ratio in the core region of $P_{\text {ext }} / P_{0} \approx 1 / 3$.

Figure 28 presents the distribution of intrinsic brightness temperature of the jet components. Note that $T_{b \text {,int }}$ is measured at distances ranging from several parsecs to a few kiloparsecs from the core (see Fig. 28, right), where the knots are generally optically thin. The distribution peaks at $T_{b \text {, int }} \sim 2 \times 10^{9} \mathrm{~K}$ for the quasar knots and $T_{b \text {, int }} \sim 6 \times 10^{7} \mathrm{~K}$ for the BL Lac knots, while for the radio galaxies the temperatures are evenly distributed between these values. The maxima should be adjusted to slightly higher temperatures, since for $30 \%$ of the quasar and BL Lac components we have obtained only upper limits to the Doppler factors. Comparison of these brightness temperatures with the intrinsic equipartition brightness temperature of the optically thick part of the jet $\left[T_{b}\right.$,int $\left.\sim(2-5) \times 10^{10} \mathrm{~K}\right]$, thought to depend weakly on source parameters (Readhead 1994; Lähteenmäki \& Valtaoja 1999), implies a faster drop of the intrinsic temperature from the compact core region to the more extended structure in the BL Lac jets. One possible explanation for a lower intrinsic brightness temperature is the presence of a stronger magnetic field (Readhead 1994). This would lead to more severe radiative losses and weaker jet components relative to the core in the BL Lac objects and perhaps a lower intrinsic brightness temperature in the cores as well.

In Figure 29 we plot the average Doppler factor versus the average viewing angle for all superluminal components (left panel), and the average viewing angle versus the intrinsic half opening angle of the jet for each source (right panel). The heavy crosses indicate the average position of the quasars, BL Lac objects, and radio galaxies, with the size of each cross equal to the $1 \sigma$ uncertainty of the parameters. Although each subclass has a large scatter around the average values and statistically the difference in the parameters of the quasars and BL Lac objects is negligible, the points form a continuous sequence on a three-dimensional plot of $\delta, \Theta_{0}$, and $\theta$ with quasars $\left(\langle\delta\rangle=23 \pm 11,\left\langle\Theta_{0}\right\rangle=2.6 \pm\right.$ $\left.1^{\circ} .9, \theta=0.5 \pm 0.3\right)$ blending into BL Lac objects $(\langle\delta\rangle=13.5 \pm$
6.7, $\left.\left\langle\Theta_{0}\right\rangle=4^{\circ} .4 \pm 33^{\circ} 0, \theta=0^{\circ} .6 \pm 0^{\circ} .4\right)$ continuing into radio galaxies $\left(\langle\delta\rangle=2.8 \pm 0.9,\left\langle\Theta_{0}\right\rangle=19^{\circ} .5 \pm 3.2, \theta=3^{\circ} .2 \pm 0.5\right)$.

\subsection{Accelerating/Decelerating Flow}

According to the $\chi^{2}$ test, one component in the radio galaxy 3C 120, five components in two BL Lac objects, and 13 components in six quasars exhibit a statistically significant change in the proper motion with time, corresponding to acceleration/deceleration with distance from the core. Therefore, in 9 out of 15 sources the apparent speed of individual components varies. Moreover, al-
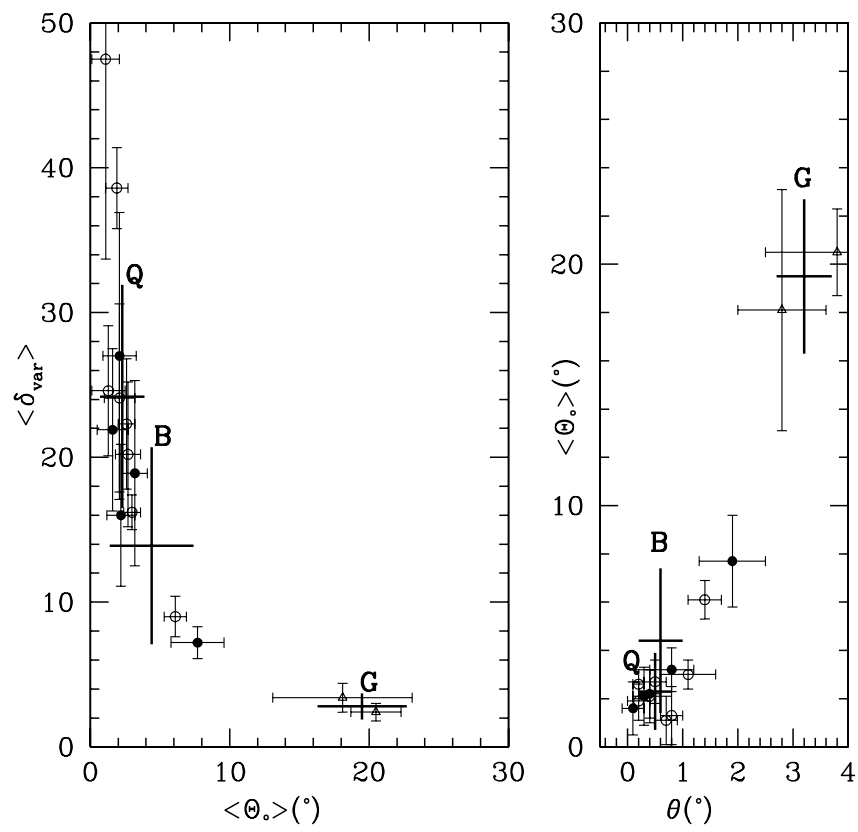

Fig. 29.-Left: Average Doppler factor vs. average viewing angle of the jets. Right: Average viewing angle vs. intrinsic half opening angle of the jets. In both panels the thick crosses indicate the average parameters and their standard deviations for each subclass. 

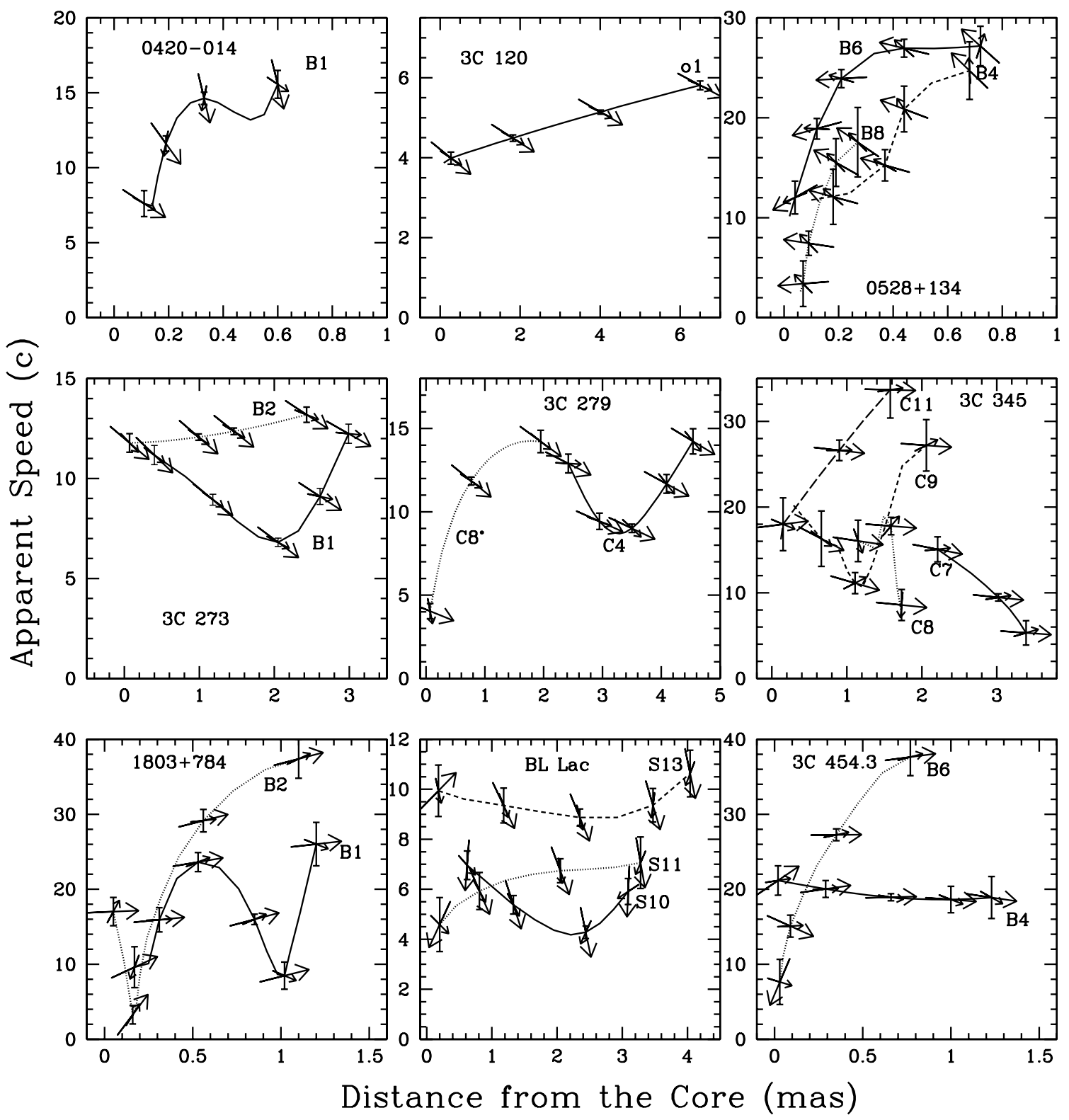

FIG. 30.-Apparent speed vs. distance from the core, as defined by a polynomial approximation, for accelerating/decelerating knots (Table 6). Short arrows represent the direction of the apparent velocity, and long arrows indicate the local jet direction; both are derived from the best-fit polynomial. Error bars for the apparent speed are computed using the uncertainties of the polynomial coefficients.

though 86 knots are classified as moving ballistically, $\sim 38 \%$ have trajectories that we are unable to fit well by a polynomial of any order, causing us to suspect nonballistic motion for these as well. Figure 30 shows the evolution of apparent velocity, including direction along the jet, derived from the best polynomial approximation of component positions across epochs for knots with a detected change in apparent speed. We have analyzed the results to search for a general trend in the variation of apparent speed with distance from the core. For each component, we compare the instantaneous apparent speed at different distances from the core with the average (indicated in Table 5) and assign a value representing the apparent speed at each angular distance of +1 if the velocity is higher than the average, -1 if it is lower than the average, and 0 if it equals the average. Then we construct the distribution of such changes along the jet using the derived viewing angle for each component (Tables $8-10$ ), which allows deprojection of observed distances from the core. The result is shown in Figure 31, in which the distribution above the $x$-axis represents accelerating components and the distribution under the $x$-axis indicates decelerating components at corresponding deprojected distances from the core. Figure 31 reveals that at distances larger than $\sim 5 \mathrm{pc}$ from the core an increase of apparent speed is more common. However, there are some jet components that undergo alternating periods of both acceleration and deceleration. The latter could be a signature of helical motion (e.g., Denn et al. 2000; Tateyama \& Kingham 2004) or pinch instabilities that cause the jet cross section, pressure, and Lorentz factor to oscillate with distance from the core (e.g., Gómez et al. 1997).

The cause of the accelerations could be bending combined with the selection of objects with high Doppler factors such that the mean angle to the line of sight of the region near the core is less than optimal for superluminal motion. Statistically, such jets are more likely to bend away from the line of sight, thus increasing their apparent speeds with distance from the core. Alternatively, the acceleration could be physical, caused by considerably higher energy density in relativistic particles than in rest 


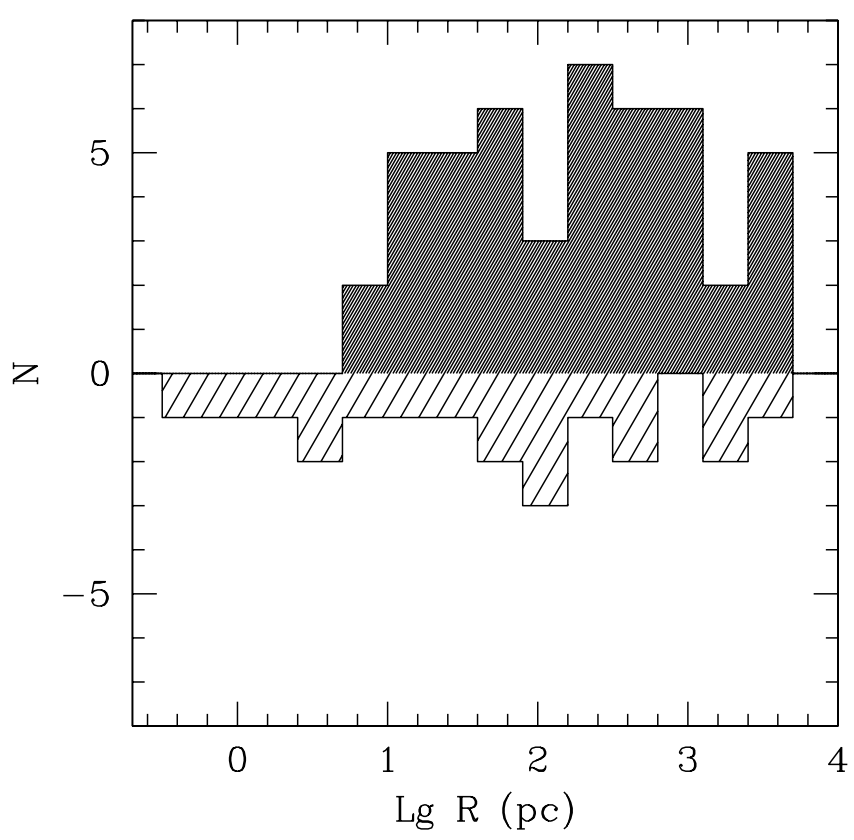

Fig. 31.-Distribution of change of the instantaneous apparent speed relative to the average apparent speed with distance from the core (deprojected). A value of +1 is assigned if the instantaneous apparent speed is higher than the average, and -1 if it is lower (see the text).

mass (Daly \& Marscher 1988) or magnetic acceleration (Vlahakis \& Königl 2004).

\subsection{Forward and Reverse Shocks}

Our data reveal the coexistence of both very fast and much slower (but moving) knots in the jets of the majority of the sources in the sample (see Fig. 16). Diversity in the apparent speeds of jet features might reflect intrinsic variations in the pattern speed of disturbances in the jet flow. In the shock wave model for interpretation of the radio light curves and features
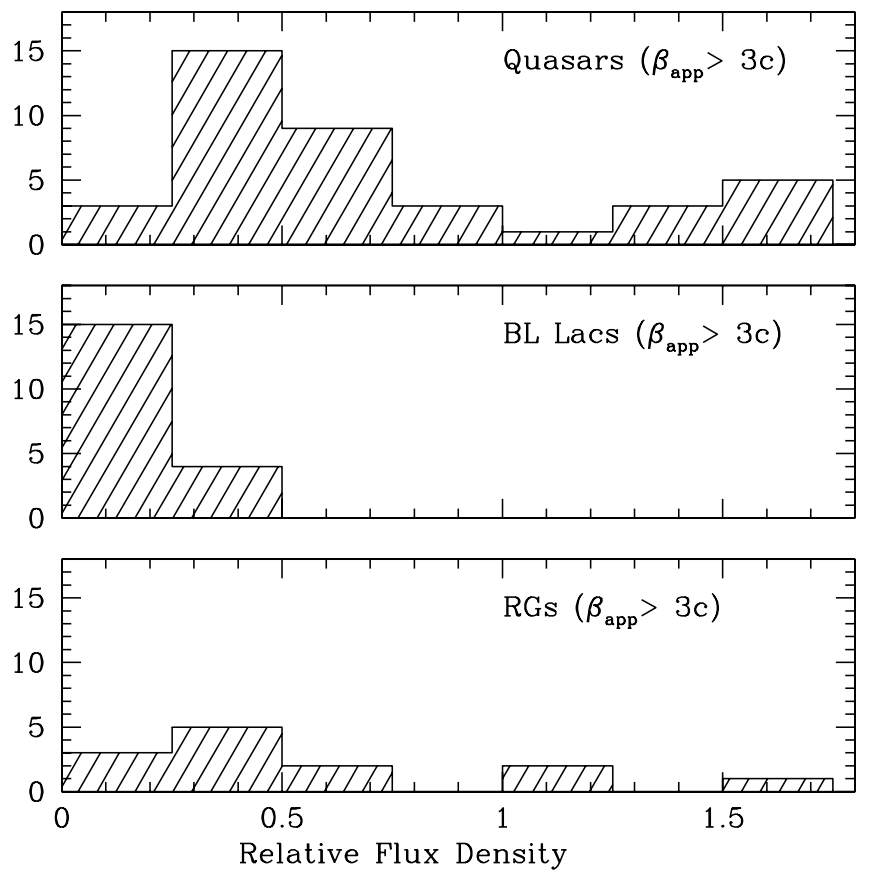

propagating down a relativistic jet, a disturbance such as an increase in velocity or energy flux in a jet can create both forward and reverse shocks (e.g., Hughes et al. 1991). Both move away from the central engine, but the reverse shock has a lower velocity than does the forward shock. In this context fast jet features can be associated with the forward shock, and slow-moving knots with the reverse shock. Analysis of the brightness of these features might reveal a prevalence of different types of shock waves in the jets for different classes of AGNs.

For each knot in Table 5 (except knots classified as trailing features; see $\S 6.6$ ) we have computed the parameter $F_{\text {rel }}=$ $S_{\max } /\left\langle S_{\mathrm{A} 0}\right\rangle$, which characterizes the flux of a knot relative to the core, where $S_{\max }$ is the maximum observed flux density of the knot and $\left\langle S_{\mathrm{A} 0}\right\rangle$ is the average flux density of the core over epochs. Figure 32 shows the distributions of the derived values of this parameter for fast- $\left(\beta_{\mathrm{app}}>3 c\right)$ and slow- $\left(\beta_{\mathrm{app}} \leq 3 c\right)$ moving knots in the quasars, BL Lac objects, and radio galaxies. The separation into fast and slow features is not strictly defined, and the distributions do not change significantly if the dividing velocity is in the range from $2 c$ to $4 c$. The distributions of $F_{\text {rel }}$ of fast knots in the quasars and BL Lac objects are different at a $99.5 \%$ level of confidence $\left(\right.$ dof $=5$ ) according to the $\chi^{2}$ test. The brightness of $\sim 50 \%$ of the fast knots in the quasars is comparable to the brightness of the core $\left(F_{\text {rel }} \gtrsim 0.5\right)$, while the brightness of fast knots in the BL Lac objects never exceeds half the brightness of the core, and the distribution peaks at $F_{\text {rel }} \leq \frac{1}{4}$. The distributions of $F_{\text {rel }}$ in the radio galaxies cannot be classified due to the small number of objects.

The distributions for slow-moving knots indicate the presence of two populations: bright $\left(F_{\text {rel }} \geq 1\right)$ and faint $\left(F_{\text {rel }} \leq 0.5\right.$ for the quasars and radio galaxies and $F_{\text {rel }}<1$ for the BL Lac objects). The population of bright slow knots consists of jet features with subluminal apparent speeds that most likely represent stationary shocks in the jets. This population is most prominent in the quasars. The population of faint slow knots is prominent in the BL Lac objects, including 39\% of all identified jet features, while in the quasars such features comprise only $7 \%$ of detected knots. About $70 \%$ of faint slow knots in the BL Lac objects have fluxes
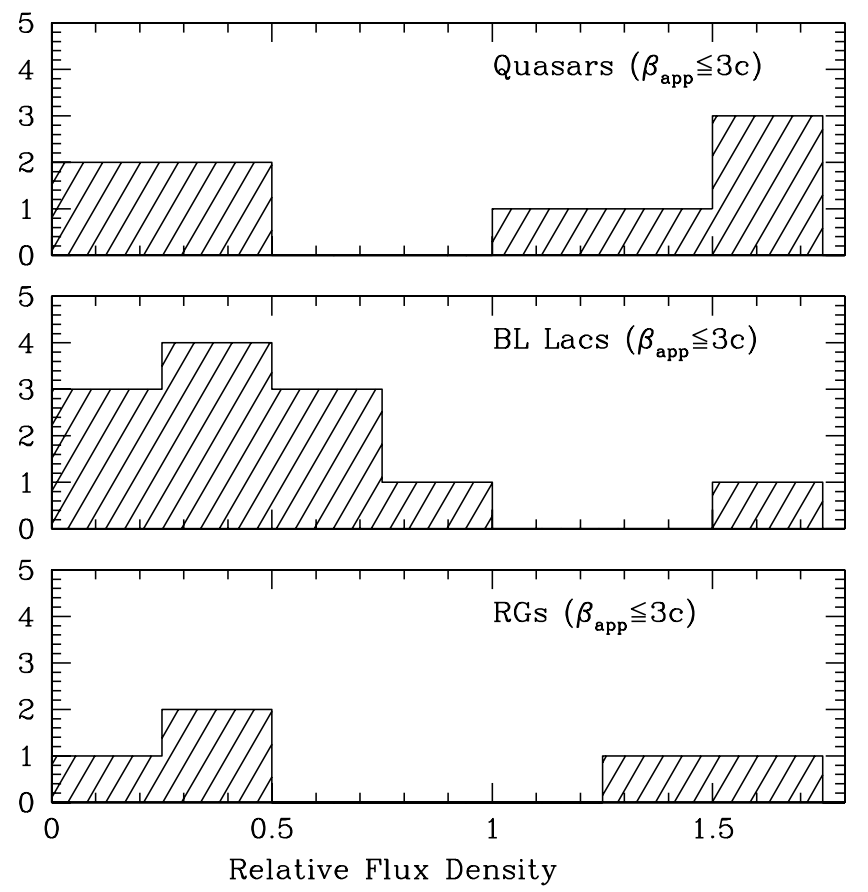

FIG. 32.-Distributions of the flux density of the jet components relative to the core for fast-moving knots (left) and for slow-moving knots (right). 
within $25 \%-100 \%$ of the flux of the core, significantly brighter than fast knots in these objects.

If fast knots represent forward shocks and slow knots correspond to reverse shocks, then the forward shock is stronger in the quasars and, perhaps, the radio galaxies, while the reverse shock dominates in the jets of BL Lac objects. A reverse shock will be strong relative to its corresponding forward shock when the disturbance is prolonged such that the faster flow enters the rear of the shock structure over an extended period of time. Otherwise, only the forward shock will be prominent, and the knot will have a Lorentz factor only slightly $(\lesssim 6 \%)$ less than that of the forward shock front (e.g., Sokolov et al. 2004). BL Lac objects might therefore have more prolonged disturbances of lower amplitude than those in quasars. If jets possess spine-sheath structure (Laing et al. 1999), then an alternative explanation for the above effect could be that the power of high- $\Gamma$ spines in BL Lac objects is lower than that in quasars. The proposal that the nature of shock waves in quasars is different from that in BL Lac objects has been suggested by Wardle et al. (1994), who interpreted the evolution of the flux and polarization of the quasar 3C 345 as forward shocks that have greater speed than the underlying jet. This is in contrast to the BL Lac object OJ 287, studied by Cawthorne \& Wardle (1988), who modeled the polarization, kinematics, and X-ray variability as reverse shocks, which are slower than the underlying jet. Our data support the idea that this could be a key difference between the two classes of blazars.

\subsection{Trailing Shocks}

Numerical hydrodynamic simulations (Agudo et al. 2001; Aloy et al. 2003) show that the interaction of disturbances in the jet flow with the underlying jet and/or the external medium can play a significant role in the variability of the jet emission. In particular, multiple conical shocks can form behind a strong shock wave propagating down the jet. These trailing shocks appear to be released in the wake of the primary superluminal component rather than ejected from the core. The simulations show that the ratio between the apparent velocities of the main and trailing components is a function of distance along the jet: the closer to the core a trailing component first appears, the slower it moves, although for a given trailing component the simulations predict deceleration with distance from the core. Trailing components are oblique shocks in three-dimensional models and should possess different polarization properties from the leading shock. Gómez et al. (2001) identified several knots in the jet of the radio galaxy 3C 120 as having the characteristics of trailing shocks. Our sequences of high-resolution VLBA images reveal a number of jet features in different sources that have properties matching those expected for this phenomenon. These are (see Fig. 16): c1 and $\mathrm{c} 2$ behind knot $\mathrm{C} 1$ in the radio galaxy $3 \mathrm{C} 111$; o 2 behind $\mathrm{o} 1$ found previously by Gómez et al. (2001) in 3C 120; b1 and b2 following $\mathrm{B} 1$ and $\mathrm{B} 2$, respectively, in the quasar $3 \mathrm{C} 273$; c9 behind $\mathrm{C} 9$ in $3 \mathrm{C} \mathrm{345}$; b5 and b6 following B5 and B6, respectively, in CTA 102; and b3 behind B3 in 3C 454.3. Components c2 (3C 111), b2 (3C 273), and b5 and b6 (CTA 102) emerge from bright knots at some distance from the core (see Fig. 16), while the remainder are already trailing bright features from the first epoch of our observations. All of them have apparent speeds less than the corresponding main component and exhibit different EVPAs than the fast feature (see Fig. 17). Although our observations do not allow us to check every trailing component for deceleration, a decrease in apparent speed is pronounced for $\mathrm{c} 1$ and $\mathrm{o} 2$ in 3C 111 and 3C 120, respectively, and can be inferred for b5 in CTA 102 (see Fig. 33). For b5 in CTA 102 a second-

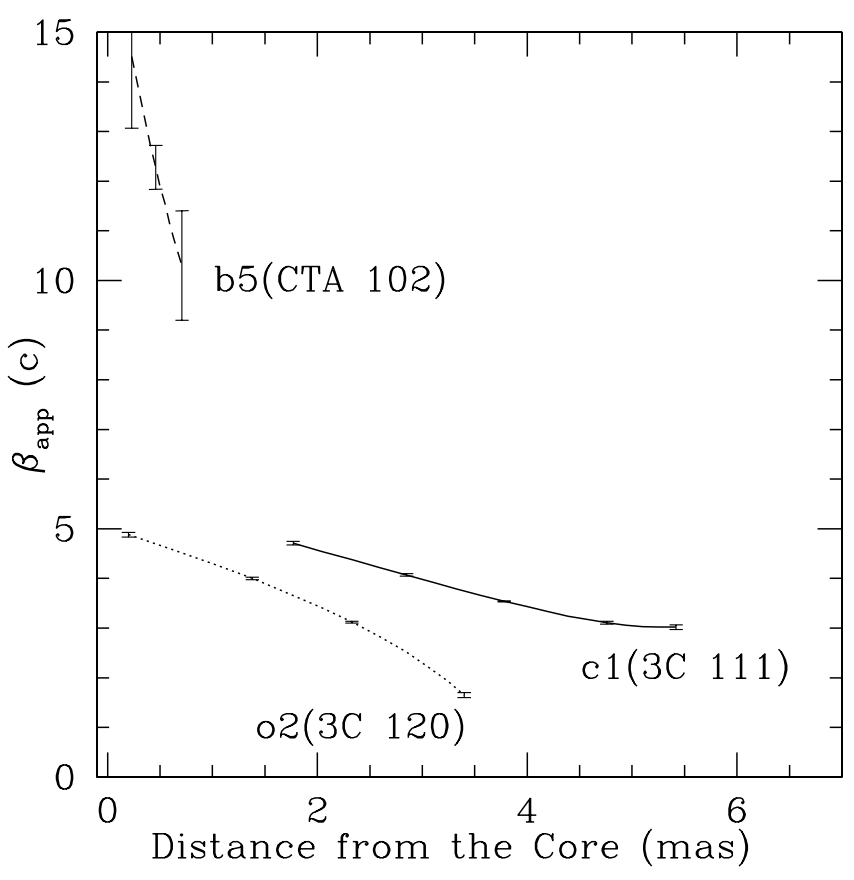

FIG. 33.-Apparent speed vs. distance from the core, as defined by a polynomial approximation, for trailing components observed at $>10$ epochs; error bars for the apparent speed are computed using the uncertainties of the polynomial coefficients

order polynomial does not satisfy the $\chi^{2}$ test but improves the goodness of fit to the data significantly.

We characterize each trailing component by three parameters: $\beta_{\text {app }}$ is the average apparent speed listed in Table $5, \beta_{\text {app }} / \beta_{\text {app }}^{M}$ is the ratio of apparent velocities of the trailing component to the corresponding main component, and $R$ is the average deprojected distance from the core at which a trailing component is detected in our data $(R$ is calculated using $\langle R\rangle$ indicated in Table 5 and the jet parameters given in Table 11). Figure 34 (left) demonstrates that trailing components form in the wake of bright knots having values of $\Gamma$ extending from 5 to $>20$, and different values of $\beta_{\text {app }} / \beta_{\text {app }}^{M}($ all of them $<1)$ are observed for similar Lorentz factors. Figure 34 (right) shows that there is an increase of the apparent speed of trailing components with distance from the core (coefficient of correlation 0.71). According to Agudo et al. (2001), trailing components represent pinch waves excited by the main disturbance, and an increase of their speed at larger distance reflects acceleration of the expanding jet. Our data shown in Figure 34 imply such an acceleration of the underlying jet that appears not to depend strongly on the Lorentz factor of the main disturbance, consistent with the conversion of internal energy into bulk kinetic energy that accompanies expansion.

Figure 34 (left) shows a possible inverse correlation between $\beta_{\text {app }} / \beta_{\text {app }}^{M}$ and the Lorentz factor of the main disturbance (coefficient of correlation -0.40 ). The correlation is most likely the result of selection effects. Detection of a trailing component close to a leading shock with a high Lorentz factor is complicated by the stretching of the longitudinal size of the main component in the observer's frame by a factor $\propto \Gamma$ owing to light-travel time delays. The detection of trailing components that lag greatly behind a main disturbance with a low Lorentz factor is hampered by difficulties in associating with confidence the trailing components to the leading knot. For this reason, many of the subluminal or quasi-stationary features detected near the core (A components) in the majority of sources in the sample could represent a superposition of many trailing components formed behind a number of 

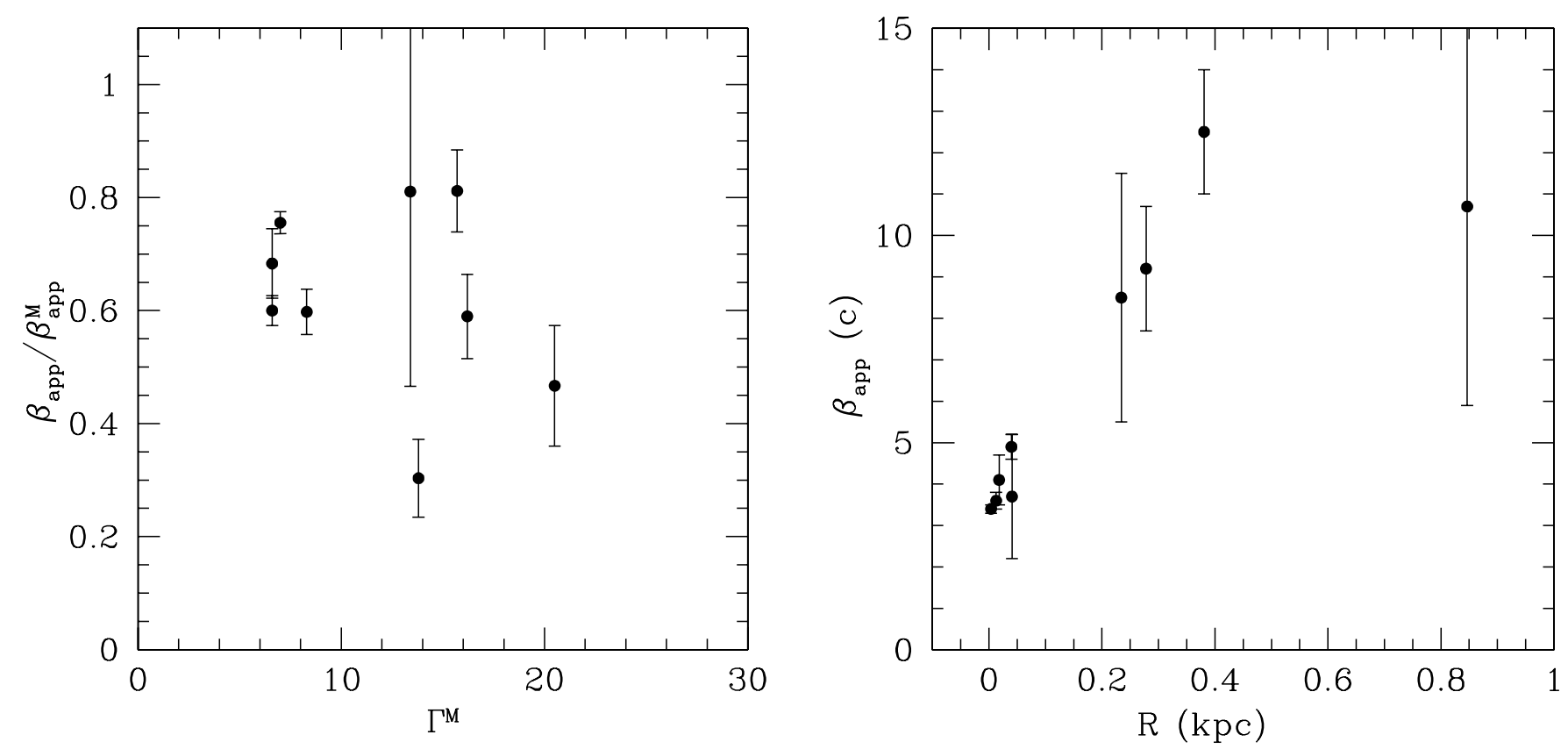

FIG. 34.-Left: Relative apparent speed of trailing components vs. Lorentz factor of the main disturbance. Right: Apparent speed of trailing components vs. deprojected distance from the core.

superluminal features. This possibility is valid only if the direction of the jet "nozzle" changes between ejections, since a second major disturbance passing through a trailing shock would destroy it.

Kellermann et al. (2004) have found that there is a systematic decrease in $\beta_{\text {app }}$ with increasing wavelength, which they suggest results from sampling different parts of the jet structure at different frequencies. Although this might be the case for average apparent speeds derived from surveys at different wavelengths, for individual sources with superluminal components detected at similar distances at different frequencies, the effect can be caused by the structure containing the leading perturbation plus slower trailing shocks being unresolved at the longer wavelengths. Per-

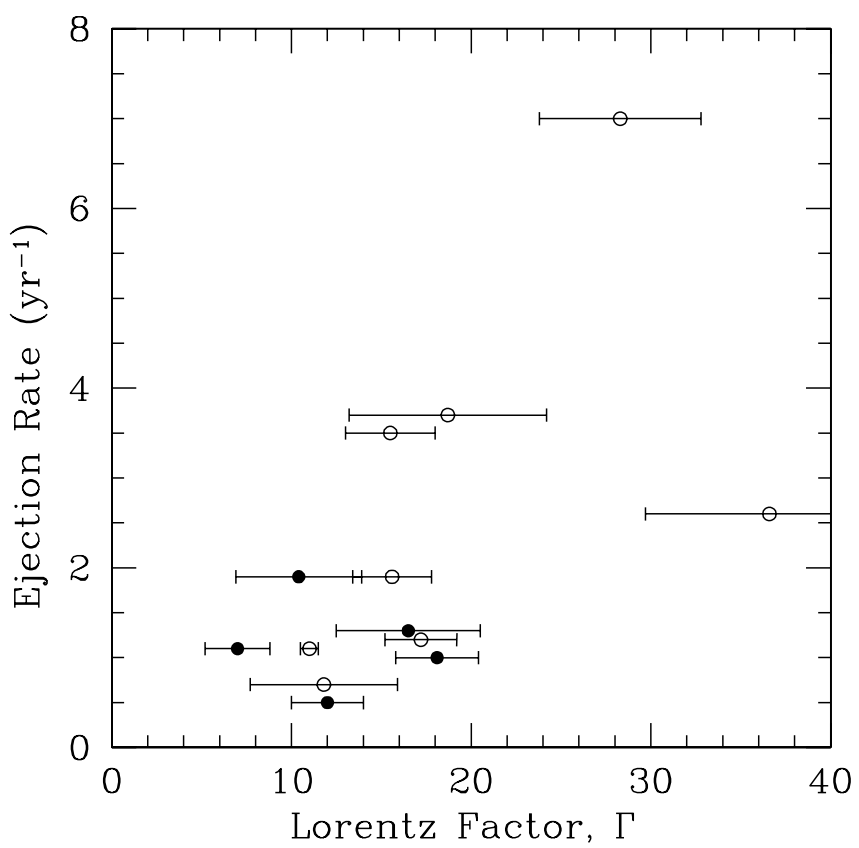

FIG. 35.-Rate of superluminal ejections in the rest frame of the AGN vs. average jet Lorentz factor. haps the difference in the proper motions of the polarized and total intensity components B8p and B8 noted in the quasar $0528+134$ (see Table 5) is an example of this effect, with the polarized intensity image (which reveals the leading compact component) playing the role of the finer resolution of shorter wavelength observations.

\subsection{Frequency of Superluminal Ejections}

Our monitoring is unique in terms of the number of blazars observed in a regular manner at high angular resolution over 3 years. The excellent time coverage allows us to determine the rate of superluminal ejections, thought to be controlled by activity in the central engine. We list in Table 11 the rate $f_{\mathrm{ej}}$ (multiplied by the time dilation factor $1+z$ ) of superluminal ejections over 3 successive years based on the results given in Table 5. In Figure 35 we plot the ejection rate versus the average jet Lorentz factor for blazars. There is a trend suggesting a positive correlation between $f_{\mathrm{ej}}$ and $\Gamma$ (coefficient of correlation 0.5 ). The formal $t_{\zeta, \nu}$ test rejects the hypothesis that there is no relation between $f_{\mathrm{ej}}$ and $\Gamma$ at a confidence level of $\zeta=0.05$. However, the trend could be an artifact of the bias toward highly Doppler boosted objects in our sample. Nevertheless, blazars form the largest class of identified $\gamma$-ray sources (Hartman et al. 1999), for which a connection between $\gamma$-ray events and radio jet activity has been found (Jorstad et al. 2001a). If nonthermal flares are associated with ejections of new superluminal knots, as seems to be the case at millimeter wavelengths (Savolainen et al. 2002), then we would expect $\gamma$-ray light curves to reflect the rate of ejections. In this case, the possible correlation between the Lorentz factor and the ejection rate would imply that the most variable $\gamma$-ray sources (in terms of major flares per year) should possess the highest Lorentz factors. This can be tested by $\gamma$-ray light curves of blazars obtained by the Gamma-Ray Large Area Space Telescope (GLAST; expected to begin operation in 2007), along with VLBI monitoring of the radio jets.

\section{SUMMARY}

In this paper we have presented the entire data set collected during a 3 year program of monitoring AGNs using the VLBA 
and have discussed the main results relating to the kinematics of jets. The sequences of images reveal short-timescale (for some sources shorter than 2 months) variability of the jet structure and even more rapid variability in polarization. This program illustrates the importance of intensive monitoring for understanding jet physics in superluminal radio sources.

We have measured the apparent speed of 106 features in the inner jets (within 4 mas of the core) of two radio galaxies, five BL Lac objects, and eight quasars from 1998 March to 2001 April. Superluminal apparent speeds occur in $80 \%$ of the knots, $26 \%$ of which show statistically significant deviations from ballistic motion. The majority of nonballistic components undergo an increase of apparent speed with distance from the core, although local decelerations are observed in some cases alongside the general acceleration. This could be the result of physical accelerations or from the selection of sources whose angles to the line of sight are less than $\sin ^{-1}(1 / \Gamma)$ near the core and closer to this value farther out. Many of the jets contain both very fast and much slower moving features, which might be explained as forward and reverse shocks, respectively. It appears that fast features are pronounced in the jets of the quasars, while slower moving knots dominate in the BL Lac jets. This suggests a different nature for the main disturbances seen in the jets of the two classes of blazars.

The properties of $11 \%$ of the superluminal components are consistent with the characteristics of trailing shocks expected to form in the wake of strong disturbances in the flow. Trailing components emerging from bright knots farther down the jet have a faster apparent speed than those generated near the core, while a given trailing feature most likely decelerates along the jet. According to the numerical simulations by Agudo et al. (2001), the trailing components are caused by the triggering of pinch modes by the main disturbance. The jet accelerates close to the core as internal energy is converted into bulk kinetic energy, leading to trailing shocks being faster farther downstream.

In four sources (3C 120, OJ 287, 3C 345, and CTA 102) the behavior of the jet components appears to be affected by interaction with the external medium (see the Appendix). For each source a number of components decelerate, change trajectory, brighten in total and polarized flux, and undergo a rotation of the EVPA at the same distance from the core along an edge of the jet. This implies the existence of gas clouds a few parsecs to $>1 \mathrm{kpc}$ (deprojected) from the central engine, intermediate between the locations of the very dense clouds of the broad-line regions and more rarefied clouds of the narrow-line regions.

Using measurements from the VLBI images of parameters such as flux density, apparent speed, and size of components, we have estimated Lorentz and Doppler factors, as well as viewing angles, for superluminal knots and the opening angle of each jet. This is a new method to define jet parameters, based on the assumption that the decay in flux of the superluminal components is caused by radiative losses rather than by cooling from expansion and is subject to light-travel delays. We demonstrate that at high radio frequencies these assumptions are most likely correct. The derived parameters of the jets indicate that in our sample the quasars have the highest Doppler factors $(\delta)$ and smallest viewing $\left(\Theta_{0}\right)$ and opening $(\theta)$ angles, while the two radio galaxies possess significantly lower Doppler factors, larger viewing angles, and wider opening angles despite their blazar-like radio properties. This implies that in the three-dimensional parameter space $\left(\delta, \Theta_{0}, \theta\right)$ the radio galaxies, BL Lac objects, and quasars in our sample occupy different regions. The regions of the quasars and BL Lac objects partly overlap, while the radio galaxies are significantly distinguished from the blazars in all three pa- rameters. Since our sample is not a complete one, the major differences suggested by these results need to be investigated further with larger, complete samples. The inferred relationship between the half opening angle and the Lorentz factor agrees with the expectation of gasdynamical models that predict smaller values of $\theta$ for higher Lorentz factors and a dependence of $\theta$ on the ratio of the external and internal jet pressure. The best approximation to the relation is very close to $P_{\text {ext }} / P_{0} \sim 1 / 3$. This does not, however, exclude collimation by magnetic pinching, which might produce a similar $\theta-\Gamma$ relation.

We have estimated the intrinsic brightness temperatures of jet components in the quasars, BL Lac objects, and radio galaxies on parsec scales, obtaining averages of $1.1 \times 10^{9}, 5.5 \times 10^{7}$, and $3.5 \times 10^{9} \mathrm{~K}$, respectively. Comparison of these values with the equipartition brightness temperature of the optically thick part of the jets, $T_{b \text {,int }} \sim(2-5) \times 10^{10} \mathrm{~K}$, suggests a stronger magnetic field in the BL Lac objects.

There is a possible positive correlation between the Lorentz factor of the jet and ejection rate of superluminal components for the blazars. This can be tested with the $\gamma$-ray light curves that will be measured by the GLAST mission.

This material is based on work supported by the National Science Foundation under grants AST 00-98579 and AST 04-06865. J. L. G. acknowledges support from the Spanish Ministerio de Educación y Ciencia and the European Fund for Regional Development through grant AYA2004-08067-C03-03. The VLBA is a facility of the National Radio Astronomy Observatory, operated by Associated Universities, Inc., under cooperative agreement with the National Science Foundation. This research has made use of data from the University of Michigan Radio Astronomy Observatory, which is supported by the National Science Foundation and by funds from the University of Michigan. We are grateful to the referee for the thorough, thoughtful, and useful criticism.

\section{APPENDIX}

\section{NOTES ON INDIVIDUAL SOURCES}

3C 66A: The radio jet consists of a bright, polarized core with weak, diffuse extended structure (Fig. 1). The set of images allows us to trace components $\mathrm{C} 1, \mathrm{C} 2, \mathrm{C} 3$, and $\mathrm{C} 4$ at many epochs (Fig. 16). They are characterized by moderate velocity, $2 c-5 c$, typical of those measured in BL Lac objects at lower frequencies (Gabuzda et al. 1994). Component $\mathrm{C} 1$ is probably feature $\mathrm{C}$ from Jorstad et al. (2001b), where it was thought to be stationary. The centroids of A1 and A2 appear to move toward the core at mildly superluminal speeds; however, these are extended features, and hence the shifts in position could be due to changes in their brightness distributions. This is supported in the case of A2, whose proper motion makes an angle of $45^{\circ}$ with the line between the core and the feature's centroid. Figure 16 shows possible identifications of very weak components B4, B6, and B7. The designation of knots follows that of Jorstad et al. (2001b); Figure 16 includes their data, marked by triangles. The $\mathrm{B}$ knots move at a high apparent speed, $>20 c$. The reality of this faster motion is supported by a region of enhanced polarized intensity that progresses rapidly downstream at the same epochs as when the high apparent speed is detected in the total intensity images (see Fig. 36). It appears that the more slowly moving $\mathrm{C}$ components and highly polarized, fast $\mathrm{B}$ components coexist in 


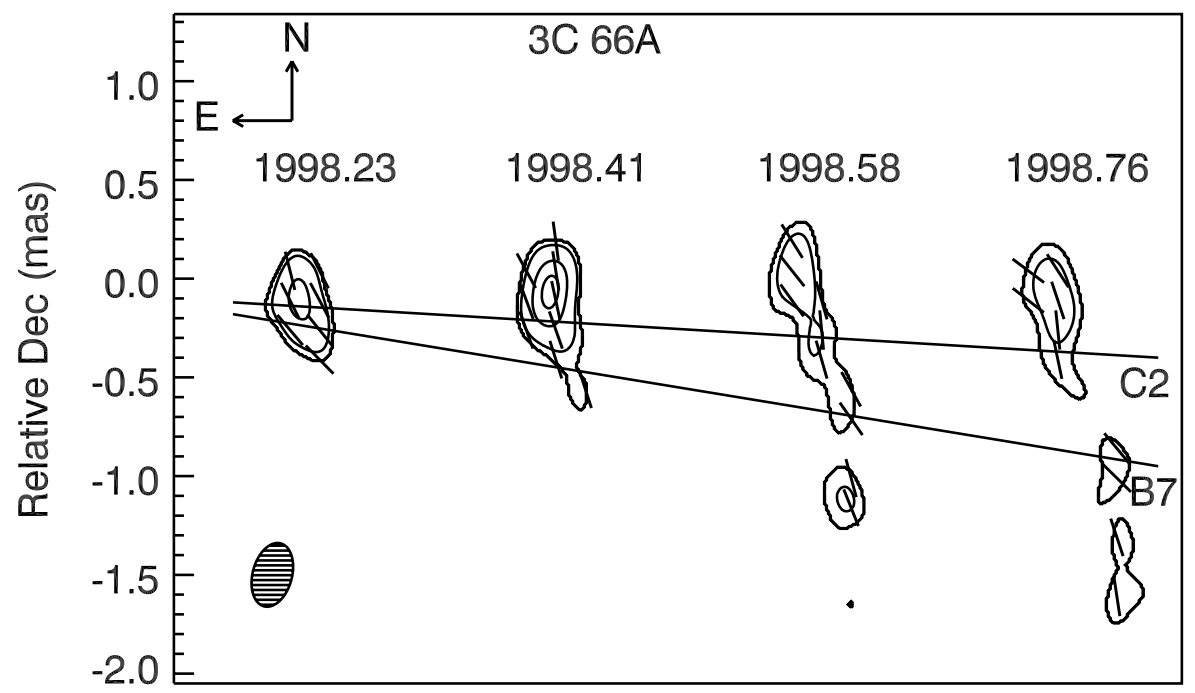

FIG. 36.-Polarized intensity images of $3 \mathrm{C} 66 \mathrm{~A}$ at four successive epochs when the highly superluminal component $\mathrm{B} 7$ is detected. The polarized intensity peak is $19.3 \mathrm{mJy}_{\text {beam }}{ }^{-1}$. The size of the beam is indicated in Table 1 . Contour levels increase by a factor of 2 , with the lowest contour corresponding to $8 \%$ of the peak polarized intensity. The line segments show the direction of the electric vectors. The solid lines indicate the motion of components B7 and C2 on the total intensity images.

the jet. One of possible physical explanations for this dichotomy is a double-shock structure, with the faster forward shock being weaker than the slower reverse shock (see $\S 6.5$ ).

3C 111: The innermost jet of this galaxy exhibits strong activity. At least seven moving components (B1-B7; see Fig. 16) can be identified inside 1 mas of the core. These knots move ballistically at a typical apparent speed of $\sim 3 c$. There are two bright features near the core, A1 and A2, that are stationary according to the $\chi^{2}$ test. The most prominent feature of the jet is an extended, polarized blob northeast of the core. The feature appears to be associated with a major outburst observed at $90 \mathrm{GHz}$ in 1996 January (Alef et al. 1998), at $37 \mathrm{GHz}$ in 1996 March, and at $22 \mathrm{GHz}$ in 1996 August (Teräsranta et al. 2004). The first ejection around this time appears to have occurred earlier than the derived epoch of ejection $T_{0}$ (see Table 5), since complex structure of the core region was detected already in a $43 \mathrm{GHz}$ VLBI map obtained in 1996 July (Alef et al. 1998). This implies slower motion of the disturbance near features A1 and A2, which agrees with the description of the structure by Alef et al. (1998) as "a mix of dimming and flaring of stationary and moving emission components." In our observations we identify the brightest part of the outburst remnant as $\mathrm{C} 1$, which moves ballistically at a higher apparent speed than that of the innermost components. Knots $\mathrm{c} 1$ and $\mathrm{c} 2$ detected behind $\mathrm{C} 1$ seem to belong to the same disturbance. At the last three epochs the parameters of $\mathrm{C} 1, \mathrm{c} 1$, and $\mathrm{c} 2$ are obtained after tapering the images by reducing the weighting of the data from the longest baselines and are not used to compute the jet velocities.

0420-014: The parsec-scale jet of this quasar is strongly dominated by the VLBI core, which has a complex polarization pattern (see Figs. 3 and 17), implying multicomponent substructure in the core region. We detect two moving components in the innermost part of the jet. Knot B1 has a slower motion near the core, $\sim 7 c$ (Fig. 30), and accelerates significantly beyond 0.2 mas as its trajectory turns from $-100^{\circ}$ to $-175^{\circ}$ (Fig. 18). The bright component $\mathrm{B} 2$ traced inside 0.15 mas from the core moves ballistically along $\Theta \sim-72^{\circ}$ at an apparent speed similar to that derived for B1 near the core. The behavior of the knots is consistent with the common curved trajectory for jet components found by Britzen et al. (2000).
3C 120: Our observations continue to trace components $d, h, l$, $\mathrm{o} 1$, and $\mathrm{o} 2$ detected in Gómez et al. (2001), and we identify new components $\mathrm{t}, \mathrm{u}, \mathrm{u} 1$, and $\mathrm{v}$ (Fig. 16). Components $\mathrm{d}$ and $\mathrm{h}$ move ballistically with the same apparent speed found previously, while 1 has decelerated since earlier epochs. Components o1 and 02 represent the front section of the major disturbance designated in Figure 17 as the "o complex." Component ol brightens in total and polarized flux at $\sim 3.4$ mas, similar to the behavior seen for $\mathrm{h}$ and 1 (Gómez et al. 2001). After flaring, $\mathrm{o} 1$ and $\mathrm{o} 2$ diverge in their behavior: o1 accelerates and 02 decelerates (Fig. 16). Such a development is expected if 02 is a trailing component that forms in the wake of the accelerating knot o1, which represents the major disturbance in the flow (Gómez et al. 2001). The most prominent feature at later epochs is $t$, which evolves in the same manner as 1 . The components have nearly equal proper motions, $2.17 \pm 0.06$ ( 1 ; Gómez et al. 2001) and $2.04 \pm 0.08 \mathrm{mas} \mathrm{yr}^{-1}(\mathrm{t})$, and similar trajectories (Fig. 18). They both undergo a flare in total and polarized flux at a location between 2.5 and 3.5 mas from the core, and the EVPAs of both rotate during the flare (Fig. 37). Gómez et al. (2000) explained these properties as the results of an interaction between the jet and an interstellar cloud. The behavior of component $t$ is consistent with this, which suggests that it interacts with the same cloud. However, there is a significant shift $(\sim 0.6$ mas $)$ between the projected positions of the peaks of the flares (see Fig. 37). Most likely, the shift is a consequence of the slightly more southern trajectory oft (Fig. 18). For a viewing angle of $\sim 21^{\circ}$ and an opening angle of $\sim 4^{\circ}$ (see Table 11), the shift by 0.6 mas gives a lower limit to the size of the cloud of $1 \mathrm{pc}$ at a deprojected distance from the VLBI core of $\sim 7-8 \mathrm{pc}$.

0528+134: Components B 3 and B4 detected in Jorstad et al. (2001b) are seen during the early epochs of our monitoring, and four new components are ejected later (Fig. 16). All components have similar trajectories, with a sharp bend to the north beyond 0.5 mas from the core, as previously noted by other authors (e.g., Britzen et al. 1999). The projected trajectories of the knots are shifted relative to each other (see Fig. 18), which suggests different angles of ejection. This is supported by a difference in the apparent speed near the core, from $>10 c$ for the fastest and most eastern components to $\sim 3 c$ for the most northern component. 


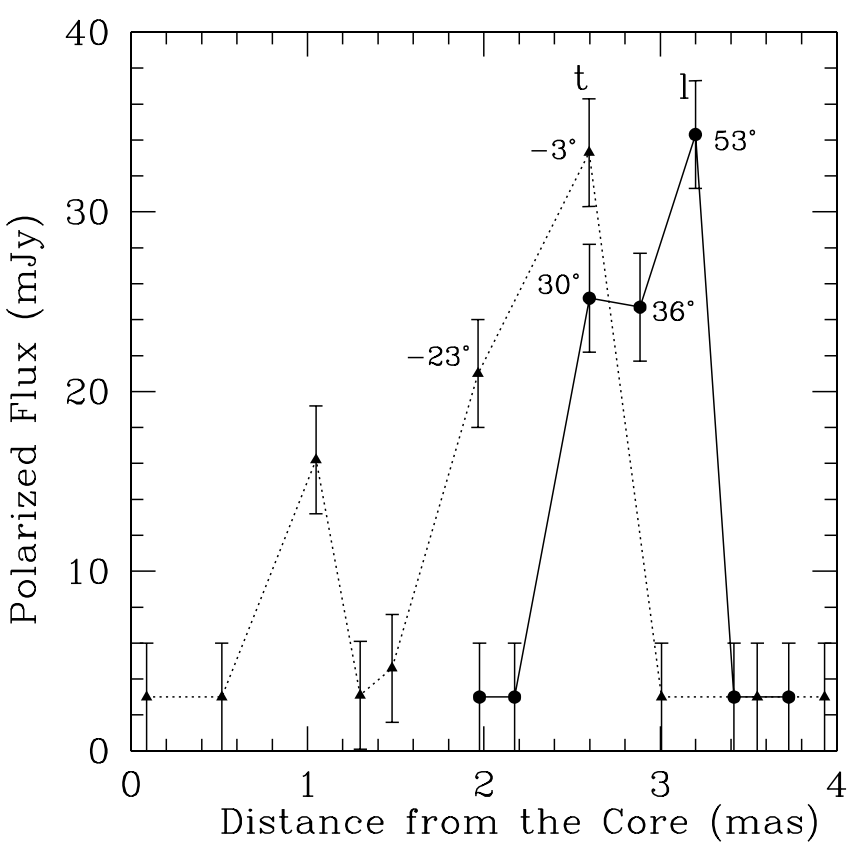

FIG. 37.-Polarized flux density of the knots 1 (solid line) and $\mathrm{t}$ (dotted line) in the radio galaxy $3 \mathrm{C} 120$ at different distances from the core. The numbers indicate values of the EVPA.

We find progressive acceleration in at least three components (Fig. 30). The most northern knot, B8, which is strongly polarized and the brightest feature in the jet (Fig. 17), shows significantly different motion of the total and polarized intensity peaks. The polarized region, B8p, moves ballistically ahead of the main concentration of total intensity, B8. Table 5 lists the parameters of B8 and B8p separately. We suggest that B8p represents a leading shock of a strong disturbance with complex structure (see $\S 6.6$ ).

OJ 287: The jet appearance changes considerably during the observations (Fig. 6), largely due to the brightness variability of three prominent jet features, A1, A2, and A3 (Fig. 17). On average, A1 and A2 separate from the core at subluminal speeds, although significant fluctuations relative to linear motion are observed (see Fig. 38). Component A2 might be associated with $\mathrm{C} 1$, classified by Jorstad et al. (2001b) as a stationary feature. Table 5 gives the parameters of A 2 based on our data alone and also based on these data combined with those of Jorstad et al. (2001b) under the assumption that $\mathrm{A} 2$ and $\mathrm{C} 1$ are the same feature. The latter is listed as $\mathrm{A} 2 *$ in Table 5 . The addition of the previous data decreases the proper motion by a factor of $\sim 2$, and in both cases the proper motion does not exceed zero at the $2 \sigma$ level. This suggests that the feature may fluctuate about a stationary position rather than translate downstream. We identify several fast-moving components in the jet. Knots B4-B7 (the designation continues the scheme of Jorstad et al. [2001b]) have very short lifetimes of $\sim 1 \mathrm{yr}$ at $43 \mathrm{GHz}$ and can be resolved only after they have passed A2 (see Fig. 17). However, a notable brightening of A2 occurs at the epochs when a B component likely reached A2 (see Fig. 16). The B components show high superluminal speeds that are slightly slower than those of knot K3/U3 in Homan et al. (2001). Knot K3/U3 is observed to accelerate, and its motion at $22 / 15 \mathrm{GHz}$ is measured $\sim 2$ times farther from the core than the locations of the B components in our images.

A distinguishing feature of the jet is component A3, the formal solution for the motion of which is upstream, toward the core. This motion is especially pronounced in the likely event that A3 is associated with C2 identified by Jorstad et al. (2001b), in

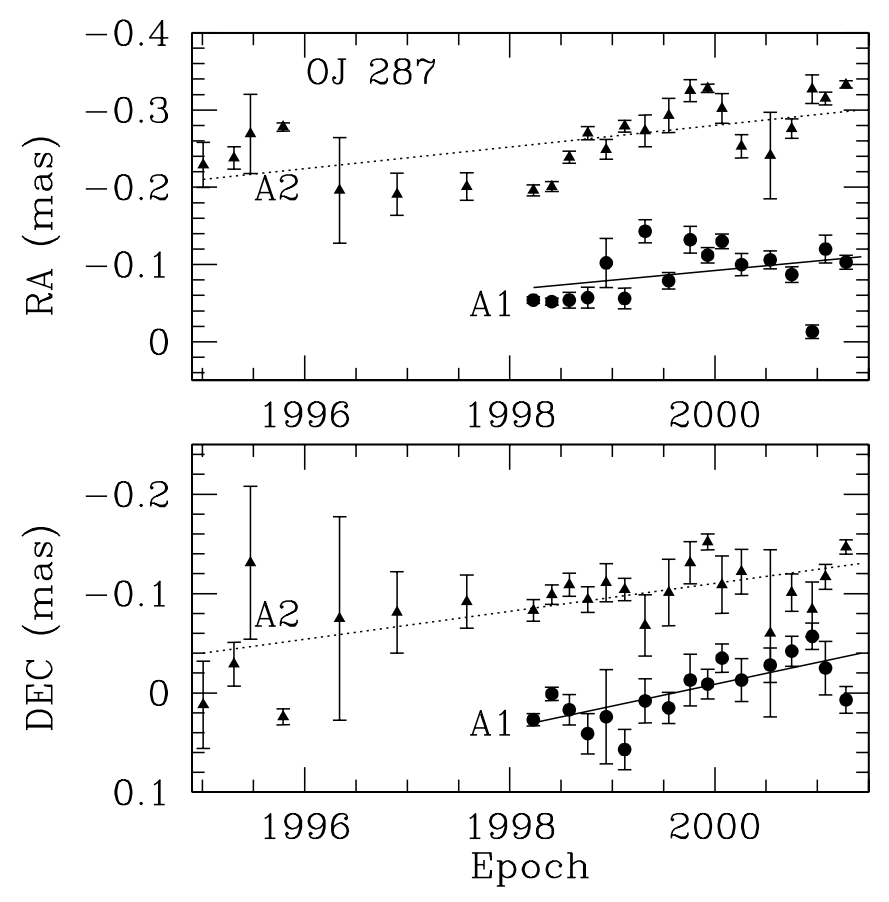

FIG. 38.- Relative right ascension and declination vs. epoch for components A1 (circles) and A2 (triangles) in OJ 287. The lines show a straight-line approximation to the temporal behavior.

which case A3 also undergoes a significant change in position angle relative to the core (Fig. 18). The latter can be explained by swinging/precession of the jet nozzle or by a translation of the core, either of which agrees with changes in trajectory of components near the core from $\Theta \sim-90^{\circ}$ for B1 (Jorstad et al. 2001b) to $\Theta \sim-115^{\circ}$ for B6. The alignment between the innermost jet direction and the position of component A3 suggests that the feature is the result of interaction between the jet flow and the external medium. The upstream apparent motion of A3 might then trace the location of an interstellar cloud (projected on the sky) when the intrinsic position angle of the jet changes. In this case, the dramatic fading of the feature toward the end of our observations (see Fig. 6) could be connected with an intrinsic change of the inner jet direction (component B6 has the most southern projected position angle during 1995-2000) so that the jet flow misses the cloud. This interpretation agrees with the findings of Tateyama \& Kingham (2004). These authors used the Radio Reference Frame Image Database and determined that the projected position angle of the inner jet of OJ 287 rotated clockwise by $\sim 30^{\circ}$ in $8 \mathrm{yr}$, in agreement with a ballistic precession model if the velocities of the components decrease beyond $\sim 1$ mas from the core. We suggest that interaction with an extragalactic cloud might cause the deceleration of components. From the derived parameters of the jet (see Table 11), we place the cloud at a deprojected distance of $\sim 90 \mathrm{pc}$ from the core. Acceleration of K3/U3 detected by Homan et al. (2001) takes place between 0.2 and 1 mas from the core and reflects an increase in the apparent speed beyond $\mathrm{A} 1$ and $\mathrm{A} 2$ and before $\mathrm{A} 3$ located at $\sim 1$ mas.

3C 273: The most prominent features in the images (see Fig. 7) are an unpolarized core and strongly polarized moving knots B1 and B2, which both brighten at $\sim 0.8$ mas (Fig. 39). Each component becomes strongly polarized just as it leaves the core region ( $\sim 0.3$ mas from the core; Fig. 17), while the core has a low polarization (comparable with the noise level). The similarity of the EVPA and fractional polarization at 7 (brightest polarized feature), 3 , and $1.3 / 0.85 \mathrm{~mm}$ suggests a low fractional 


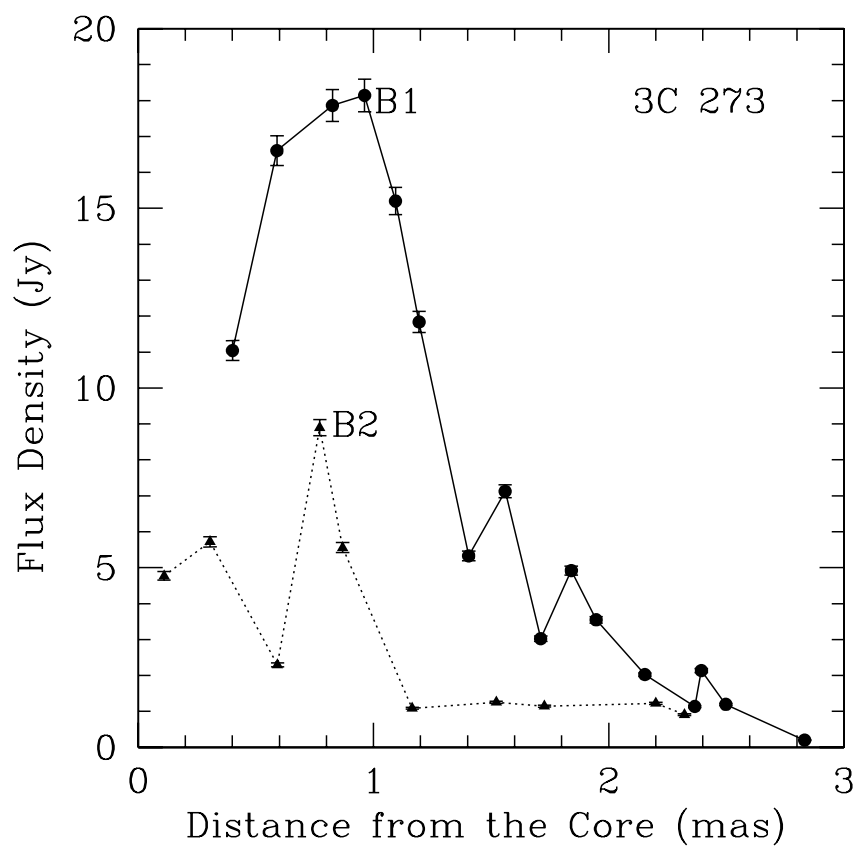

FIG. 39.-Total flux density of knots B1 (solid line) and B2 (dotted line) in the quasar 3C 273 at different distances from the core.

polarization in the core at the high frequencies, where Faraday effects are not important. This implies that the core has intrinsically low polarization, which might be caused by a strongly turbulent magnetic field on scales smaller than the $7 \mathrm{~mm}$ synthesized beam. After they flare, B1 and B2 fade dramatically and expand both along and transverse to the jet direction. This results in the formation of new features $b 1$ and $b_{s}$ related to $B 1$, and $b 2$ related to $\mathrm{B} 2$ (Fig. 16). The $\mathrm{b}$ components might form in the interaction of relativistic shocks connected with features B1 and B2 and the underlying jet flow. These components should have lower apparent speed than the main disturbances (Agudo et al. 2001), but this is not the case for $b_{s}$. Alternatively, the B components are fairly extended features in a broad jet, and the $b$ knots might represent sections of the jet where the velocity vectors lie at different angles to the line of sight. The latter interpretation is supported by the difference in the projected trajectories of the B and $b$ components (Fig. 18) and the strong dependence between the projected position angle and apparent speed of the components, with the more northern knots being slower (Fig. 40). A substantial velocity gradient across the jet is also expected according to a model in which the underlying jet flow has a double-helix structure and a lower Lorentz factor than that of the disturbances (Lobanov \& Zensus 2001). The brightening of $\mathrm{B} 1$ and $\mathrm{B} 2$ at $\sim 0.8$ mas from the core might be connected with either (1) a change of the jet direction (a slight curvature of the projected trajectories of both components is seen in Fig. 18 at this distance), (2) an intensity peak of the threadlike pattern of the underlying flow where the local velocity vector bends toward the line of sight (Lobanov \& Zensus 2001), or (3) interaction with the external medium. However, a stable polarization direction in B2 before, during, and after the flare (see epochs 1999.76, 1999.93, and 2000.07 in Fig. 7) does not support the third possibility.

At many epochs the images contain a diffuse, highly polarized (up to $50 \%$ ) feature, $\mathrm{C} 1$, farther down the jet (see Fig. 41) and moving at an apparent speed of $\sim 7 c$. According to the time of ejection, $\mathrm{C} 1$ corresponds to knot G1 found by Jorstad et al. (2001b). If C1 and G1 are the same feature, it has maintained a nearly constant proper motion over $\sim 5 \mathrm{yr}$.

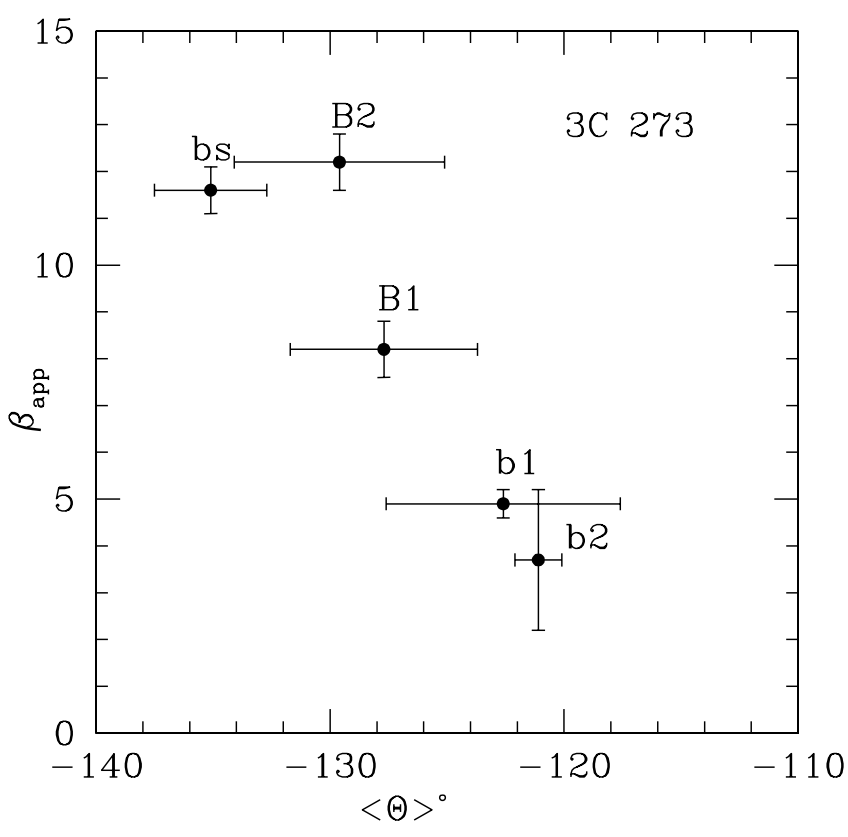

FIG. 40.-Apparent speed vs. projected position angle of superluminal components in the quasar 3C 273.

3C 279: Our data reveal the motion of at least eight components, C9-C16 (our designations continue those of Wehrle et al. 2001 ), with apparent speeds up to $17 c, 3$ times higher than previously reported velocities measured since 1991 (Wehrle et al. 2001; Homan et al. 2001; Jorstad et al. 2001b). The identification of components in Figure 16 includes results at $43 \mathrm{GHz}$ from Wehrle et al. (2001; triangles) and Jorstad et al. (2001b; squares). Components $\mathrm{C} 9-\mathrm{C} 16$ are shown in Figure 17 at the last epoch of monitoring. Jorstad et al. (2004) suggest that the remarkable change in apparent speed is caused by a shift in the direction of the jet nozzle while the Lorentz factor remains constant. This conclusion is supported by a gradual decrease of the projected position angle $\Theta$ of each successively ejected component (starting with C9). In this interpretation, the jet flow is characterized by $\Gamma \geq 20$, while $\Theta_{0}$ changes from 0.5 for components with the lowest speed ( $5.5 c$ for $\mathrm{C} 8$ found by Wehrle et al. 2001) to $1^{\circ} .6$ for components with the highest speed. It is also possible that component $\mathrm{C} 8$ accelerates (see Fig. 16) due to an increase in the Lorentz factor of the flow. We designate this possible component as $\mathrm{C} 8^{*}$; it corresponds to $\mathrm{C} 8$ before the acceleration and to $\mathrm{C} 9$ after the acceleration. As indicated in Table 6, the change in the velocity component parallel to the jet, $\dot{\mu}_{\|}$, is significant. Another method, which we apply in this paper to estimate Doppler and Lorentz factors and viewing angle for each superluminal knot (see $\S 6$ ), suggests that both the Lorentz factor and the angle between the jet axis and line of sight for $\mathrm{C} 9-\mathrm{C} 16$ are different from those of $\mathrm{C} 8$. In any event, these results are generally consistent with viewing angle playing the major role in the change of apparent speed.

Contemporaneous to these changes in the inner jet, an increase in the apparent speed is observed for $\mathrm{C} 4$ at $\sim 3$ mas from the core at epoch $\sim 1998.2$ (Homan et al. 2003). Our data, combined with previously published results at $43 \mathrm{GHz}$ (Wehrle et al. 2001; Jorstad et al. 2001b), show that variations in the trajectory and apparent speed of C4 occurred at least twice between 1995 and 2001. This identifies three segments (a, b, and c) of the trajectory (Fig. 18, dotted lines) for which the motion is ballistic according to a $\chi^{2}$ test. The ballistic sections of the trajectory define two epochs, $1996.1 \pm 0.1$ and $1998.07 \pm 0.03$, when the apparent 


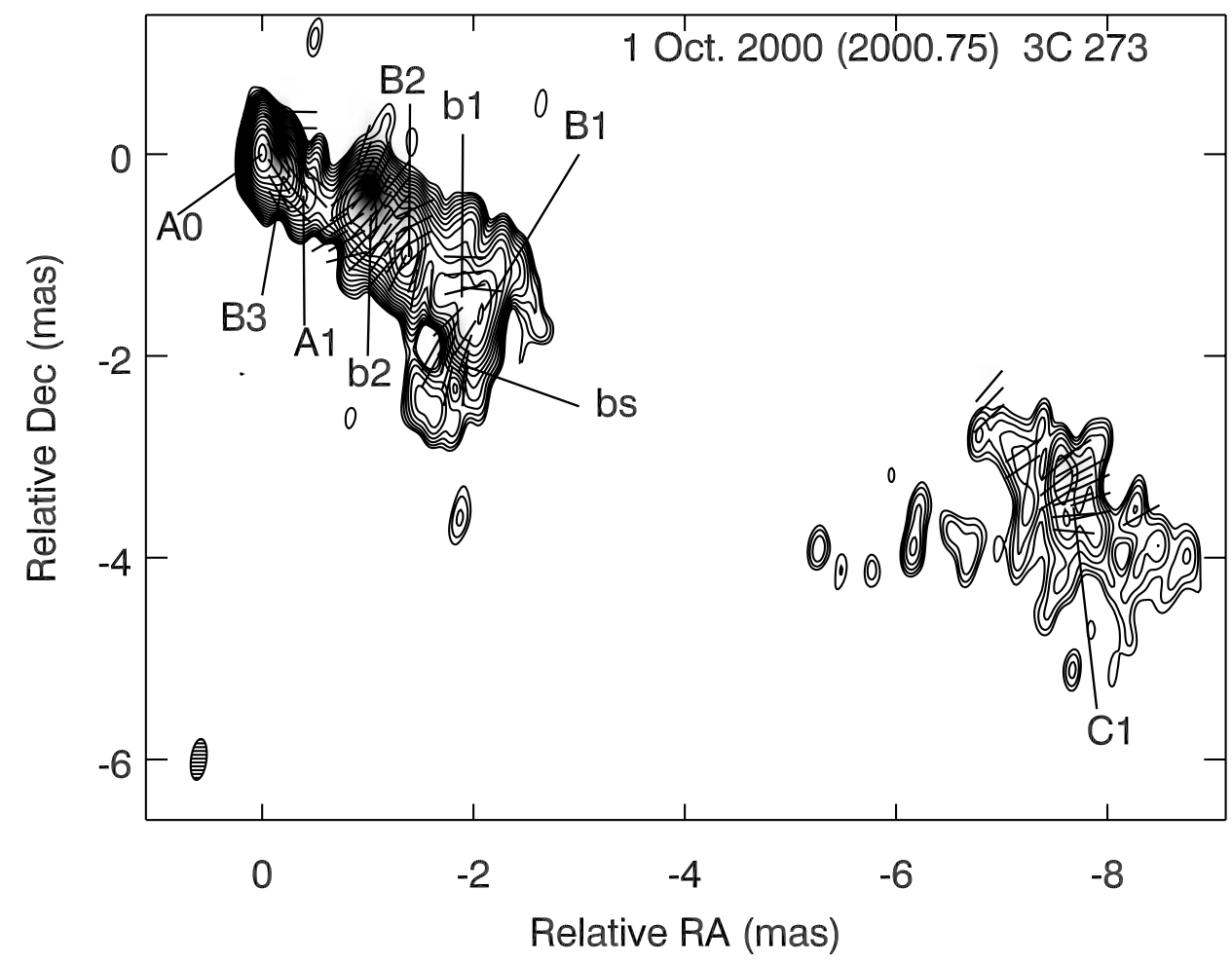

FIG. 41.-Total (contours) and polarized (gray scale) intensity images of $3 \mathrm{C} 273$. The total intensity peak is $1710 \mathrm{mJy}^{-1}$ beam $^{-1}$, the polarized intensity peak is $90 \mathrm{mJy}$ beam ${ }^{-1}$, the beam is $0.39 \times 0.14 \mathrm{mas}^{2}$ at P.A. $=-6.5$, and contours increase by a factor of $\sqrt{2}$, with the lowest contour corresponding to $0.25 \%$ of the peak. The line segments indicate the electric vector direction.

speed changes. The values of $\beta_{\text {app }}$ corresponding to these paths are given in Table 5 using designations $\mathrm{C} 4 \mathrm{a}, \mathrm{C} 4 \mathrm{~b}$, and $\mathrm{C} 4 \mathrm{c}$, respectively. Table 5 shows that $\mathrm{C} 4$ had a high apparent speed $\left(\beta_{\text {app }} \sim 13 c\right)$ in 1995 and decelerated dramatically in 1996, then accelerated again from 1998 until at least 2002 according to Homan et al. (2003). Both periods of acceleration correspond to the fading of the component, while during times of lower apparent speed C4 brightened. Such behavior may be the result of helical motion, with the radius of the helix increasing outward.

1510-089: The radio emission of this quasar is strongly core dominated with typically weak extended jet structure. Our data indicate that the quasar possesses an ultrarelativistic jet with apparent speed up to $\sim 46 c$. However, even bimonthly observations are not sufficient to state that the identification of components shown in Figures 9, 16, and 42 is unique. On the other hand, this identification is supported by the total and polarized intensity images along with the total intensity light curves at $37 \mathrm{GHz}$ (Teräsranta et al. 2004) and $14.5 \mathrm{GHz}$ (University of Michigan Radio Astronomy Observatory database). We detect three moving components: B1 (Fig. 9), B2, and B3 (Fig. 42). In all three cases we observe a similar polarization behavior in the core region when the component is emerging from the core: the core region is strongly polarized with EVPA along the jet direction (see Fig. 9). In the cases of components B2 and B3, polarized features with similar EVPA are subsequently observed propagating down the jet (Fig. 42). For all three components the time of the ejection falls on the rising branch of an outburst in the total intensity light curves (an especially prominent flare is connected with the ejection of component B2). The modeling of the jet structure shows two features, A1 and A2, that fluctuate near positions 0.14 and 0.54 mas, respectively (Fig. 16). Component A1 usually cannot be resolved from a moving component at epochs when the latter is close to A1, while A2 shifts down- stream when a moving component is approaching and turns back when the moving knot has passed by (see Fig. 16). Such a behavior of jet features formed by stationary shocks is seen in numerical simulations of jets with variable input flow velocities (Aloy et al. 2003). At many epochs, a feature, S, with flux significantly above the noise is seen southeast of the core (Fig. 17), close to the position angle of the arcsecond-scale jet (Homan et al. 2002b). On the other hand, this component is located at a distance from the core similar to that of A1 but in the opposite direction. This suggests possible artificial generation of the knot owing to modeling of an actually slightly extended core using a pointlike source. However, there is no correlation between the positions and fluxes of S and A1. The position angle of S varies within the range $71^{\circ}-168^{\circ}$, and the flux varies from 0.03 to $0.5 \mathrm{Jy}$, while the values of $\Theta$ and the flux of A1 are fairly stable (parameters of A1 and A2 in Table 5 are computed for epochs when the features are not confused with moving knots). Differences in the properties of S and A1 suggest that these features, along with $\mathrm{A} 0$, constitute a complex core region inside the $43 \mathrm{GHz}$ synthesized beam.

3C 345: At many epochs the core of this quasar is not the brightest feature of the jet (Fig. 10). The core is usually unpolarized at $43 \mathrm{GHz}$ (Fig. 17), although the fractional polarization of the component nearest to the core, A1, can be as high as $20 \%$. The latter fact suggests the low polarization of the core is intrinsic rather than caused by Faraday effects. We detect seven superluminal knots with average apparent speeds in the range $9 c-24 c$ (Fig. 16; where the component designations follow Ros et al. 2000), and at least four of these exhibit acceleration or deceleration (Figs. 18 and 30). For knots C10-C13 statistically significant acceleration is found only for $\mathrm{C} 11$. Similar acceleration was reported for components $\mathrm{C} 4$ and $\mathrm{C} 7$ by Biretta et al. (1983) and Ros et al. (2000), although beyond 2 mas from the 

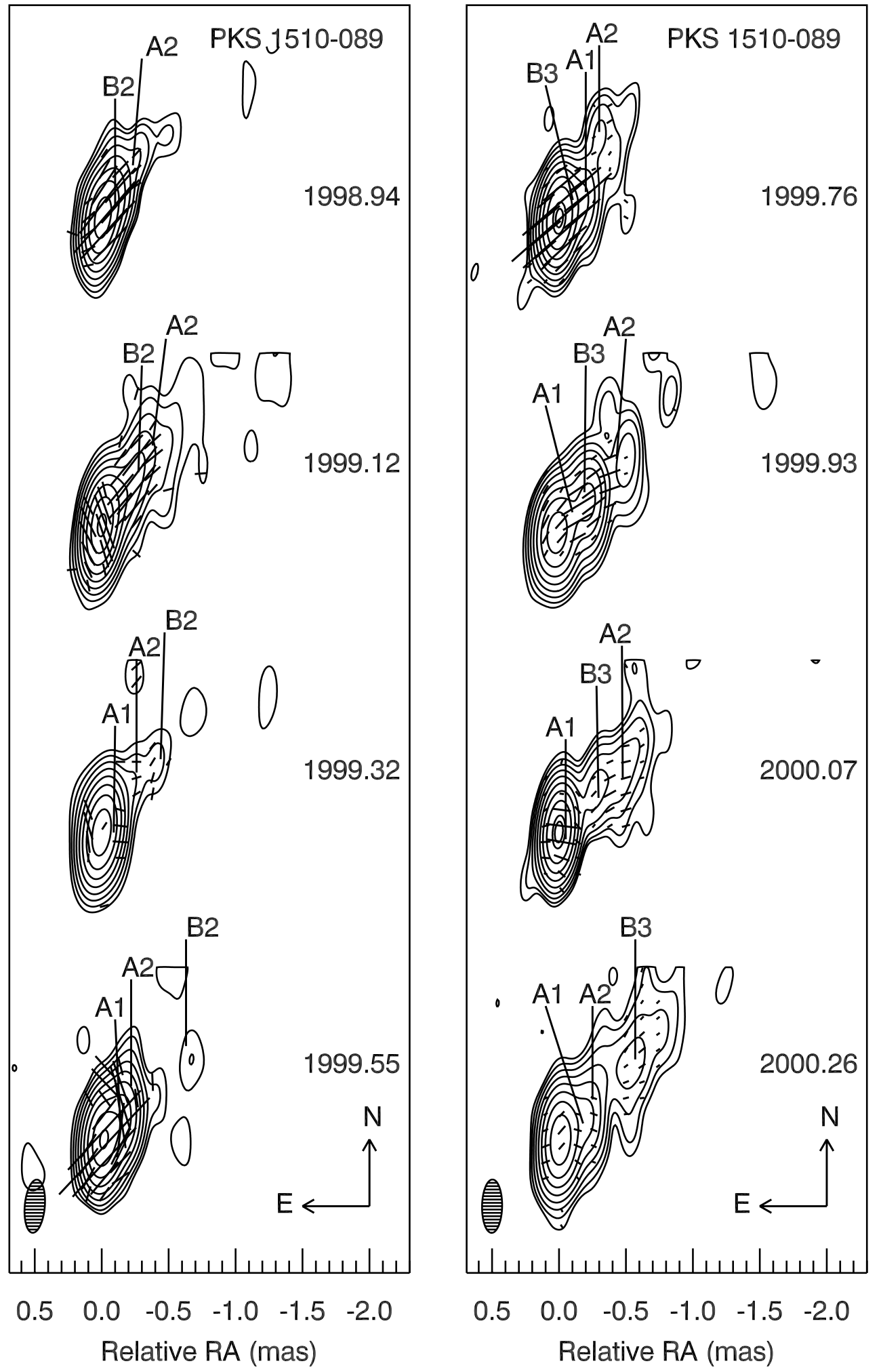

FIG. 42.- Total intensity images with polarization vectors of the quasar PKS $1510-089$ from 1998.94 to $1999.55\left(\right.$ left: $S_{\text {peak }}=1520 \mathrm{mJy}$ beam ${ }^{-1}$ and $S_{\text {peak }}^{p}=$ $107 \mathrm{mJy}$ beam ${ }^{-1}$ ) and from 1999.76 to 2000.26 (right: $S_{\text {peak }}=1700 \mathrm{mJy} \mathrm{beam}^{-1}$ and $S_{\text {peak }}^{p}=147 \mathrm{mJy} \mathrm{beam}^{-1}$ ). The beam is $0.40 \times 0.15 \mathrm{mas}^{2}$ at P.A. $=-5^{\circ}$, and contour levels are $0.3 \%, 0.6 \%, \ldots, 76.8 \%$, and $90 \%$ of the total intensity peak.

core C7 decelerates, as indicated by both $\dot{\mu}_{\|}$and interpolation of its position relative to 1996.81 as observed by Ros et al. (2000; Fig. 16, asterisks). It appears that $\mathrm{C} 8$ repeats the stages of $\mathrm{C} 7$, although it experiences an earlier and greater deceleration, possibly signifying a more complex trajectory (see Fig. 18). The change in apparent speed and the twisted trajectory are also pronounced for C9. Although the historically extrapolated position of C9 (using the average apparent speed of 15.6c) agrees well with the measured position at epoch 1996.81 (Ros et al. 2000 ), it decelerates significantly from $\sim 20 c$ near the core to $\sim 10 c$ at 1 mas and then accelerates beyond 1.5 mas. The deceleration is accompanied by brightening of the knot and rotation 


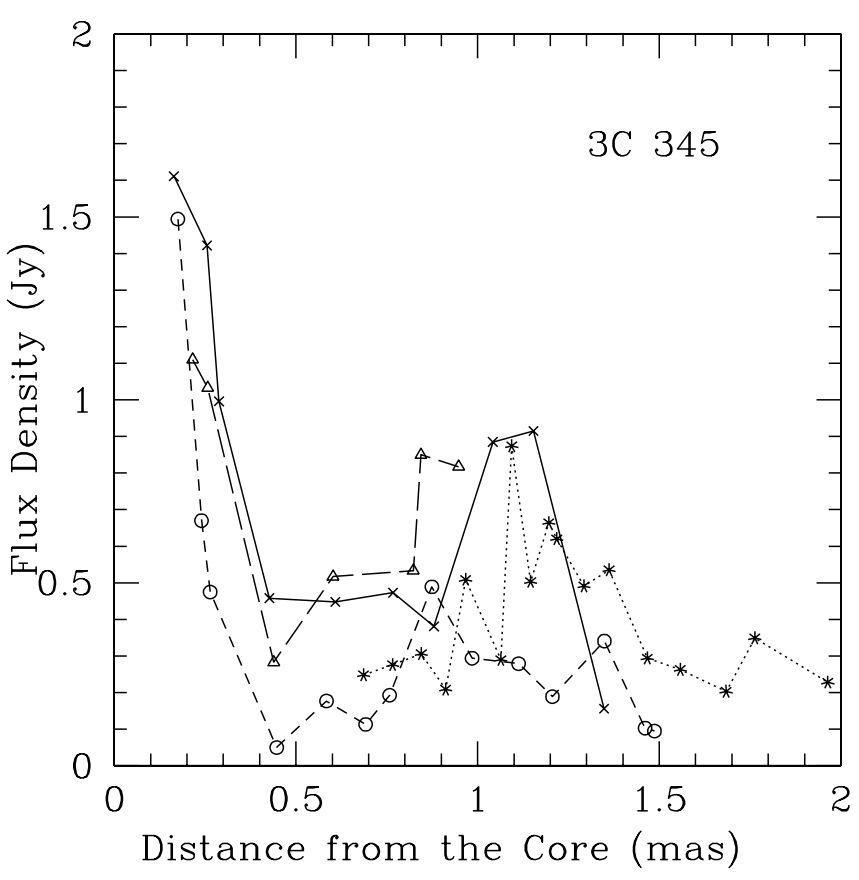

Fig. 43.-Total flux density of knots C9 (asterisks), C10 (circles), C11 (crosses), and $\mathrm{C} 12$ (triangles) in the quasar 3C 345 at different distances from the core.

of the EVPA by $\sim 50^{\circ}$, making it almost perpendicular to the jet direction (see epochs 1998.76 and 1998.94 in Fig. 10). In addition, $\mathrm{C} 9$ has the most southern position angle in the jet during the deceleration period $\left(\Theta \sim-116^{\circ}\right)$, similar to that observed for C8 by Ros et al. (2000), $\Theta \sim-122^{\circ}$. Wardle et al. (1994) interpreted a change they observed in apparent speed in terms of a flow with constant Lorentz factor along a bent trajectory. Component $\mathrm{C} 9$ expands as it propagates down the jet and leaves behind a complex structure that consists of knot $\mathrm{c} 9$ moving at an apparent speed of $\sim 9 c$ (Fig. 16) plus two extended features, $c 9 n$ and $\mathrm{c} 9 \mathrm{~s}$, that form later at the northern and southern edges of the jet, respectively (Fig. 17). The majority of moving features have a trajectory with zigzags in the north-south direction (although the net motion is to the west), an increase in flux, and rotation of the EVPA at a distance $\sim 0.8-1$ mas from the core (Fig. 43). These variations could be the result of interaction with the external medium, as suggested by Ros et al. (2000). We estimate the deprojected distance of this possible interaction as $\sim 300 \mathrm{pc}$.

1803+784: The curved jet of this BL Lac object has been interpreted as the result of helical structure (Britzen et al. 2001; Tateyama et al. 2002). Our high-resolution $43 \mathrm{GHz}$ images show at least two superluminal components near the core, B1 and B2. These follow a similar curved path (Fig. 18) and have average apparent speeds of $\sim 16 c$, with acceleration and deceleration along the trajectory (Fig. 30). A passage from deceleration to acceleration and vice versa is detected three times for B1, and locations of these transitions along the jet coincide with bends in the trajectory (compare Figs. 18 and 30). This behavior supports the hypothesis of helical structure of the jet. Gabuzda \& Chernetskii (2003) classify the jet feature $\sim 1.5$ mas from the core as a stationary knot, S. In our data this feature appears to be associated with A2 and has an upper limit to its apparent speed of $\sim 1.5 c$ (Fig. 16). No motion is detected for the bright knot A1 near the core. Tateyama et al. (2002) found that a superluminal component at $8.5 \mathrm{GHz}$ only becomes visible at a distance $\geq 1$ mas from the core. Moving component $\mathrm{C} 1$ is detected beyond $\mathrm{A} 2$ and has an apparent speed of $\sim 10 c$, similar to that found by Britzen
\& Krichbaum (1995). The fractional polarization in the jet is high. For example, A2 is polarized up to $30 \%$, with the EVPA aligned with the jet direction. In contrast, the EVPA in the core region is essentially radial (see Fig. 17), supporting the inference of a toroidal magnetic field geometry (Gabuzda 2000), but only if the viewing angle is almost $0^{\circ}$ in the immediate vicinity of the core. It is interesting to note that the viewing angle of the jet derived in $\S 6$ is indeed quite small.

1823+568: The $43 \mathrm{GHz}$ images of this BL Lac object contain bright stable features A1, A2, and A3 within 0.5 mas of the core (Fig. 16). All of these components are strongly polarized, especially $\mathrm{A} 3$, for which the fractional polarization reaches $25 \%$ (Fig. 17). Four components detected in our images, A2, A3, C2, and $\mathrm{C} 1$, can be identified with components $\mathrm{K} 4, \mathrm{~K} 3, \mathrm{~K} 2$, and $\mathrm{K} 1$, respectively, from Gabuzda \& Cawthorne (2000). The locations of A2 and A3 are slightly farther from the core than K4 and K3 (epoch 1995.58). This can be attributed to a shift in the core position at $43 \mathrm{GHz}$ relative to $22 \mathrm{GHz}$ due to opacity effects, in which case the positions of these features would be stable over a period of 5 yr. Despite excellent time coverage (Fig. 12), we do not detect motion inside 1 mas of the core in our observations. This has several possible explanations: (1) ejections of superluminal components are rare in this object, with a mean time between ejections $\geq 20 \mathrm{yr}$ in the observer's frame (see Table 5); (2) the moving components are much fainter than the stationary features; or (3) the innermost part of the jet is very close to the line of sight. However, we do detect two moving components, $\mathrm{C} 1$ and $\mathrm{C} 2$, beyond 1 mas from the core where the jet bends slightly to the west (see Fig. 17). Knot C1 has a low apparent speed of $3.8 c$ that appears to be essentially constant, since the linear extrapolation of its motion back to epoch 1995.58 agrees well (within 0.03 mas) with the position of knot K1 in the image of Gabuzda \& Cawthorne (2000). For the fastest moving feature in the jet, $\mathrm{C} 2\left(\beta_{\text {app }} \sim 8 c\right)$, extrapolation from our sequence produces a position of the component at epoch 1995.58 that is farther from the core by $\sim 0.1$ mas than was observed for K2. This implies a slower apparent speed farther from the core. No motion of $\mathrm{C} 2$ is detected in our images after it passes $\mathrm{C} 1$ (Fig. 16).

BL Lac: Our data for BL Lac have been analyzed in Stirling et al. (2003). At least three new components have been ejected during the monitoring program (Fig. 16). These, together with previously ejected knots observed by Denn et al. (2000), form a sequence of superluminal features that appear to emerge from the core ballistically along different position angles, indicating a change in direction of the jet nozzle. The trajectories of the knots exhibit curvature that is especially pronounced for components S10, S11, and S13 (Fig. 18). Despite different average apparent speeds, the components accelerate in a similar manner suggestive of a small change in the Lorentz factor and a substantial change in direction (see Table 6, Fig. 30). The core region can be represented by two pointlike features, $\mathrm{C} 1$ and $\mathrm{C} 2$ (Stirling et al. 2003; Fig. 17). In the inner jet two bright stationary knots, $\mathrm{A} 1$ and A2, are observed at all epochs (see Figs. 16 and 17).

CTA 102: The apparent velocities of the jet components in this quasar are fairly high, $\beta_{\text {app }} \sim 18 c$, in agreement with previous findings (Jorstad et al. 2001b; Rantakyrö et al. 2003). We label the newly detected moving components as B5 and B6 (Fig. 16) following the scheme of Jorstad et al. (2001b) and rename $J$ in Rantakyrö et al. (2003) as B4 based on its estimated ejection time. Components B5 and B6 have ballistic motion. However, at $\sim 0.2$ mas from the core both knots split, forming more slowly moving components $\mathrm{b} 5$ and $\mathrm{b} 6$ behind the main features. At some epochs (e.g., Fig. 17) B5 is significantly brighter than the core in both total and polarized intensity. There are two features, A1 (near 


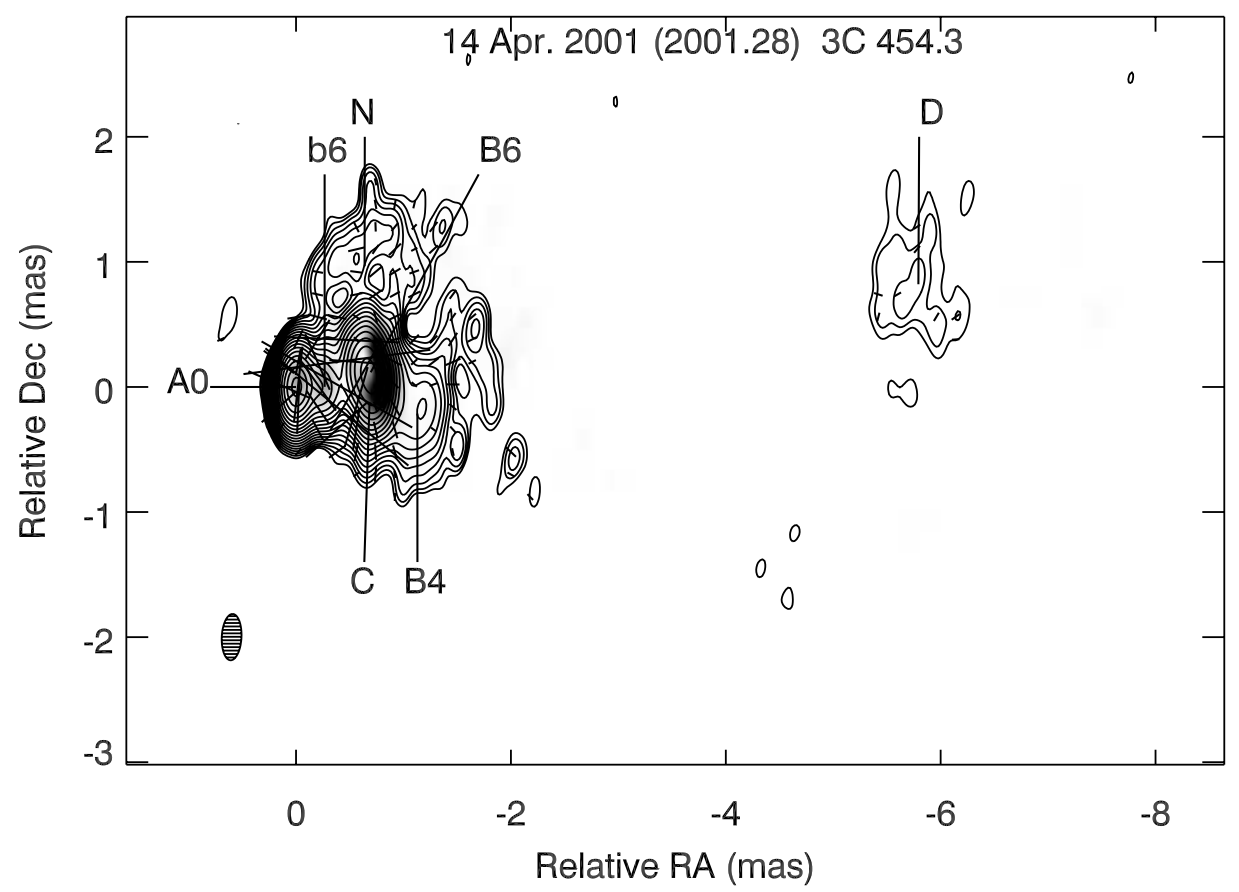

FIG. 44.-Total (contours) and polarized ( gray scale) intensity images of 3C 454.3 . The total intensity peak is $1860 \mathrm{mJy}^{-1}$ beam ${ }^{-1}$, and the polarized intensity peak is $71 \mathrm{mJy}^{\text {beam }}{ }^{-1}$. The beam is $0.31 \times 0.15$ mas $^{2}$ at P.A. $=-6^{\circ}$; contour levels increase by a factor of $\sqrt{2}$, with the lowest contour corresponding to $0.25 \%$ of the peak. The line segments indicate the polarization direction.

the core) and $\mathrm{C}(\sim 2$ mas from the core), that do not move according to a $\chi^{2}$ test. Component $\mathrm{C}$ was detected by Jorstad et al. (2001b) and classified as probably stationary, although Rantakyrö et al. (2003) associated the feature with different moving components. As noted by Jorstad et al. (2001b), C is strongly variable in brightness and located where the jet bends sharply to the south. In 1998-1999, C was bright, very diffuse, and showed a double structure that included knot c closer to the core (see Fig. 17). We detect no motion of c (Fig. 16). Both components fade dramatically by the end of the monitoring (Fig. 14) despite the approach of the growing inner part of the jet toward C. This behavior appears similar to that observed for component A3 in OJ 287 (see above), where we associate the fading of A3 with a change in the jet direction. Figure 18 shows the trajectories of B5 and B6, as well as B1 and B3 from Jorstad et al. (2001b), plus the position of $\mathrm{C}$ during both sets of observations. The location of $\mathrm{C}$ fluctuates over the range 1-1.5 mas from the core in right ascension and within 1.4-1.6 mas (to the south) in declination. The innermost jet shows a significant $\left(\sim 20^{\circ}\right)$ difference in projected position angle of B5 and B6 relative to B1-B3, suggestive of wobbling of the jet nozzle and/or change of the VLBI core position. These properties imply interaction between the moving flow and external medium at a deprojected distance $\sim 1.4 \mathrm{kpc}$ from the core that results in the formation of a diffuse feature.

3C 454.3: This quasar has a very complex structure at $43 \mathrm{GHz}$. Figure 16 plots only bright components (flux density $>0.1 \mathrm{Jy}$ ). One of the most interesting features of the jet is the highly polarized component C. At all epochs (see Figs. 15 and 17) the EVPA of the brightness peak of $\mathrm{C}$ aligns with the jet axis. No change in separation of the component from the core has been seen during either previous observations (Jorstad et al. 2001b) or our current sequence of images. However, combining the data from the two studies reveals a drift of $\mathrm{C}$ to the southwest (see Fig. 18) at an apparent speed of $\sim 1.6 c$ between 1995 and 2001 (the component defined in this way is referred to as $\mathrm{C}^{*}$ in Table 5), while the size of the knot remains fairly stable at $\sim 0.25 \pm$
0.10 mas. The absence of proper motion within each data set suggests that the observed difference in position of the feature ( $\sim 0.2$ mas in declination and $\sim 0.1$ mas in right ascension) could be caused by a displacement to the northeast of the VLBI core between 1997.6 and 1998.2. Beyond C, trajectories of moving components separate into two groups, northwestern (B3, B6) and southwestern (B4), with a difference between the projected trajectories that can reach $\sim 70^{\circ}$ (Fig. 18). This is consistent with the direction of the jet near the location of $\mathrm{C}$ lying very close to the line of sight, thereby producing large differences in projected trajectories of moving components beyond $\mathrm{C}$ even if the intrinsic trajectories differ only slightly. The proper motions that we mea-

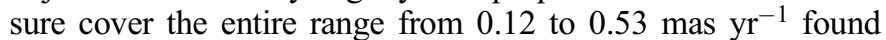
previously in the jet of 3C 454.3 (Gómez et al. 1999; Jorstad et al. 2001b; and references therein). Component B6 accelerates markedly (see Table 6). A similar acceleration is derived for a bright feature detected in 1998 beyond $\mathrm{C}$ if we associate it with B3 found by Jorstad et al. (2001b) based on its brightness, position, and time of ejection. This gives an increase in apparent speed of B3 from $6 c$ near the core to $13 c$ beyond C. Both of these components belong to the northwestern group (Fig. 18). Northwestern components appear to accelerate significantly without a change in direction of motion, while southwestern knots (e.g., B4) show mostly a change in direction (Fig 30). A diffuse feature (designated in Fig. 16 by the plus sign) is located in the northern part of the jet and appears to move after B4 passes C. Figure 17 displays component $\mathrm{S}$ to the south of $\mathrm{C}$ when $\mathrm{B} 4$ approaches $\mathrm{C}$. As observed in other objects discussed above, slower moving knots b3 and b6 trail some of the bright fast components (B3 and $\mathrm{B} 6$, respectively). Figure 44 shows knot $\mathrm{D} \sim 6$ mas from the core. This can be associated with a feature $\left(\mathrm{A}_{\mathrm{PT}}\right)$ imaged at longer wavelengths by Pauliny-Toth (1998). According to Pauliny-Toth (1998) $A_{P T}$ accelerated in 1984-1989 from $16 c$ to $30 c$ and then decelerated to $20 c$ in 1992 . Our observations suggest a possible continuation of the deceleration such that the apparent speed of the knot has decreased to $6 c$. 


\section{REFERENCES}

Agudo, I., Gómez, J.-L., Martí, J.-M., Ibáñez, J.-M., Marscher, A. P., Alberdi, A., Aloy, M.-A., \& Hardee, P. E. 2001, ApJ, 549, L183

Alef, W., Preuss, E., Kellermann, K. I., \& Gabuzda, D. 1998, in IAU Colloq. 164, Radio Emission from Galactic and Extragalactic Compact Sources, ed. J. A. Zensus, G. B. Taylor, \& J. M. Wrobel (ASP Conf. Ser. 144; San Francisco: ASP), 129

Aloy, M.-Á., Martí, J.-M., Gómez, J.-L., Agudo, I., Müller, E., \& Ibáñez, J.-M. 2003, ApJ, 585, L109

Biretta, J. A., Cohen, M. H., Unwin, S. C., \& Pauliny-Toth, I. I. K. 1983, Nature, 306, 42

Blandford, R. D., \& Königl, A. 1979, ApJ, 232, 34

Bowker, A. H., \& Lieberman, G. J. 1972, Engineering Statistics (Englewood Cliffs: Prentice-Hall)

Brandt, S. 1999, Data Analysis: Statistical and Computational Methods for Scientists and Engineers (New York: Springer)

Britzen, S., \& Krichbaum, T. P. 1995, in Proc. 10th Working Meeting on European VLBI for Geodesy and Astronomy, ed. R. Lanotte \& G. Bianco (Matera: ASI), 172

Britzen, S., Witzel, A., Krichbaum, T. P., Campbell, R. M., Wagner, S. J., \& Qian, S. J. 2000, A\&A, 360, 65

Britzen, S., Witzel, A., Krichbaum, T. P., MacSlow, T., \& Matveyenko, L. I. 2001, Astron. Lett., 27, 1

Britzen, S., Witzel, A., Krichbaum, T. P., Qian, S. J., \& Campbell, R. M. 1999 , A\&A, 341, 418

Burbidge, G. R., Jones, T. W., \& Odell, S. L. 1974, ApJ, 193, 43

Cawthorne, T. V., \& Wardle, J. F. C. 1988, ApJ, 332, 696

Daly, R. A., \& Marscher, A. P. 1988, ApJ, 334, 539

Denn, G. R., Mutel, R. L., \& Marscher, A. P. 2000, ApJS, 129, 61

Gabuzda, D. C. 2000, in Astrophysical Phenomena Revealed by Space VLBI, ed. H. Hirabayashi, P. G. Edwards, \& D. W. Murphy (Tokyo: ISAS), 121

Gabuzda, D. C., \& Cawthorne, T. V. 2000, MNRAS, 319, 1056

Gabuzda, D. C., \& Chernetskii, V. A. 2003, MNRAS, 339, 669

Gabuzda, D. C., Mullan, C. M., Cawthorne, T. V., Wardle, J. F. C., \& Roberts, D. H. 1994, ApJ, 435, 140

Gabuzda, D. C., \& Sitko, M. L. 1994, AJ, 107, 884

Gabuzda, D. C., Sitko, M. L., \& Smith, P. S. 1996, AJ, 112, 1877

Gómez, J. L., Marscher, A. P., \& Alberdi, A. 1999, ApJ, 522, 74

Gómez, J. L., Marscher, A. P., Alberdi, A., Jorstad, S. G., \& Agudo, I. 2001, ApJ, 561, L161

. 2002, VLBA Scientific Memo 30 (Socorro: NRAO)

Gómez, J. L., Marscher, A. P., Alberdi, A., Jorstad, S. G., \& García-Miró, C. 2000, Science, 289, 2317

Gómez, J. L., Martí, J. M. A., Marscher, A. P., Ibánez, J. M. A., \& Alberdi, A. 1997, ApJ, 482, L33

Greaves, J. S., et al. 2003, MNRAS, 340, 353

Hartman, R. C., et al. 1999, ApJS, 123, 79

Holland, W. S., et al. 1999, MNRAS, 303, 659

Homan, D. C., Lister, M. L., Kellermann, K. I., Cohen, M. H., Ros, E., Zensus, J. A., Kadler, M., \& Vermeulen, R. C. 2003, ApJ, 589, L9

Homan, D. C., Ojha, R., Wardle, J. F. C., Roberts, D. H., Aller, M. F., Aller, H. D., \& Hughes, P. A. 2001, ApJ, 549, 840

- 2002a, ApJ, 568, 99

Homan, D. C., Wardle, J. F. C., Cheung, C. C., Roberts, D. H., \& Attridge, J. M. 2002b, ApJ, 580, 742

Hughes, P. A., Aller, H. D., \& Aller, M. F. 1985, ApJ, 298, 301 1991, ApJ, 374, 57

Impey, C. D., \& Tapia, S. 1990, ApJ, 354, 124

Jorstad, S. G., Marscher, A. P., Lister, M. L., Stirling, A. M., Cawthorne, T. V., Gómez, J. L., \& Gear, W. K. 2004, AJ, 127, 3115
Jorstad, S. G., Marscher, A. P., Mattox, J. R., Aller, M. F., Aller, H. D., Wehrle, A. E., \& Bloom, S. D. 2001a, ApJ, 556, 738

Jorstad, S. G., Marscher, A. P., Mattox, J. R., Wehrle, A. E., Bloom, S. D., \& Yurchenko, A. V. 2001b, ApJS, 134, 181

Kantowski, R., Kao, J. K., \& Thomas, R. C. 2000, ApJ, 545, 549

Kellermann, K. I., Vermeulen, R. C., Zensus, J. A., \& Cohen, M. H. 1998, AJ, 115,1295

Kellermann, K. I., et al. 2004, ApJ, 609, 539

Lähteenmäki, A., \& Valtaoja, E. 1999, ApJ, 521, 493

Laing, R. A., Parma, P., de Ruiter, H. R., \& Fanti, R. 1999, MNRAS, 306, 513

Leppänen, K. J., Zensus, J. A., \& Diamond, P. J. 1995, AJ, 110, 2479

Lister, M. L., Kellermann, K. I., Vermeulen, R. C., Cohen, M. H., Zensus, J. A., \& Ros, E. 2003, ApJ, 584, 135

Lister, M. L., \& Marscher, A. P. 1997, ApJ, 476, 572

Lister, M. L., \& Smith, P. S. 2000, ApJ, 541, 66

Lobanov, A. P., \& Zensus, J. A. 2001, Science, 294, 128

Lovell, J. 2000, in Astrophysical Phenomena Revealed by Space VLBI, ed. H. Hirabayashi, P. G. Edwards, \& D. W. Murphy (Tokyo: ISAS), 301

Marscher, A. P., \& Gear, W. K. 1985, ApJ, 298, 114

Marscher, A. P., Jorstad, S. G., Gómez, J. L., Aller, M. F., Teräsranta, H., Lister, M. L., \& Stirling, A. M. 2002, Nature, 417, 625

Mead, A. R. G., Ballard, K. R., Brand, P. W. J. L., Hough, J. H., Brindle, C., \& Bailey, J. A. 1990, A\&AS, 83, 183

Meier, D. L., Koide, S., \& Uchida, Y. 2001, Science, 291, 84

Nartallo, R., Gear, W. K., Murray, A. G., Robson, E. I., \& Hough, J. H. 1998, MNRAS, 297, 667

Pauliny-Toth, I. I. K. 1998, in IAU Colloq. 164, Radio Emission from Galactic and Extragalactic Compact Sources, ed. J. A. Zensus, G. B. Taylor, \& J. M. Wrobel (ASP Conf. Ser. 144; San Francisco: ASP), 75

Pearson, T. J., et al. 1998, in IAU Colloq. 164, Radio Emission from Galactic and Extragalactic Compact Sources, ed. J. A. Zensus, G. B. Taylor, \& J. M. Wrobel (ASP Conf. Ser. 144; San Francisco: ASP), 17

Rantakyrö, F. T., Wiik, K., Tornikoski, M., Valtaoja, E., \& Bååth, L. B. 2003, A\&A, 405, 473

Readhead, A. C. S. 1994, ApJ, 426, 51

Readhead, A. C. S., Cohen, M. H., Pearson, T. J., \& Wilkinson, P. N. 1978, Nature, 276, 768

Roberts, D. H., Wardle, J. F. C., \& Brown, L. F. 1994, ApJ, 427, 718

Ros, E., Zensus, J. A., \& Lobanov, A. P. 2000, A\&A, 354, 55

Savolainen, T., Wiik, K., Valtaoja, E., Jorstad, S. G., \& Marscher, A. P. 2002, A\&A, 394, 851

Shepherd, M. C. 1997, in ASP Conf. Proc. 125, Astronomical Data Analysis Software and Systems VI, ed. G. Hunt \& H. E. Payne (San Francisco: ASP), 77

Sitko, M. L., Schmidt, G. D., \& Stein, W. A. 1985, ApJS, 59, 323

Sokolov, A. S., Marscher, A. P., \& McHardy, I. 2004, ApJ, 613, 725

Stirling, A. M., et al. 2003, MNRAS, 341, 405

Tateyama, C. E., \& Kingham, K. A. 2004, ApJ, 608, 149

Tateyama, C. E., Kingham, K. A., Kaufmann, P., \& de Lucena, A. M. P. 2002, ApJ, 573, 496

Teräsranta, H., \& Valtaoja, E. 1994, A\&A, 283, 51

Teräsranta, H., et al. 2004, A\&A, 427, 769

Tzioumis, A. K., et al. 1989, AJ, 98, 36

Vlahakis, N., \& Königl, A. 2004, ApJ, 605, 656

Wardle, J. F. C., Cawthorne, T. V., Roberts, D. H., \& Brown, L. F. 1994, ApJ, 437, 122

Wehrle, A. E., Piner, B. G., Unwin, S. C., Zook, A. C., Xu, W., Marscher, A. P., Teräsranta, H., \& Valtaoja, E. 2001, ApJS, 133, 297

Wills, B. J., Wills, D., Breger, M., Antonucci, R. R. J., \& Barvainis, R. 1992, ApJ, 398, 454 